TRANSACTIONS OF THE

AMERICAN MATHEMATICAL SOCIETY

Volume 366, Number 1, January 2014, Pages 159-217

S 0002-9947(2013)05951-X

Article electronically published on May 21, 2013

\title{
DIRICHLET AND NEUMANN PROBLEMS FOR PLANAR DOMAINS WITH PARAMETER
}

\author{
FLORIAN BERTRAND AND XIANGHONG GONG
}

\begin{abstract}
Let $\Gamma(\cdot, \lambda)$ be smooth, i.e. $\mathcal{C}^{\infty}$, embeddings from $\bar{\Omega}$ onto $\overline{\Omega^{\lambda}}$, where $\Omega$ and $\Omega^{\lambda}$ are bounded domains with smooth boundary in the complex plane and $\lambda$ varies in $I=[0,1]$. Suppose that $\Gamma$ is smooth on $\bar{\Omega} \times I$ and $f$ is a smooth function on $\partial \Omega \times I$. Let $u(\cdot, \lambda)$ be the harmonic functions on $\Omega^{\lambda}$ with boundary values $f(\cdot, \lambda)$. We show that $u(\Gamma(z, \lambda), \lambda)$ is smooth on $\bar{\Omega} \times I$. Our main result is proved for suitable Hölder spaces for the Dirichlet and Neumann problems with parameter. By observing that the regularity of solutions of the two problems with parameter is not local, we show the existence of smooth embeddings $\Gamma(\cdot, \lambda)$ from $\overline{\mathbb{D}}$, the closure of the unit disc, onto $\overline{\Omega^{\lambda}}$ such that $\Gamma$ is smooth on $\overline{\mathbb{D}} \times I$ and real analytic at $(\sqrt{-1}, 0) \in \overline{\mathbb{D}} \times I$, but for every family of Riemann mappings $R(\cdot, \lambda)$ from $\overline{\Omega^{\lambda}}$ onto $\overline{\mathbb{D}}$, the function $R(\Gamma(z, \lambda), \lambda)$ is not real analytic at $(\sqrt{-1}, 0) \in \overline{\mathbb{D}} \times I$.
\end{abstract}

\section{INTRODUCTION}

Let $k \geq 0$ be an integer and $0<\alpha<1$. Let $\Omega^{\lambda}(0 \leq \lambda \leq 1)$ be a family of bounded domains in $\mathbf{C}$ of $\mathcal{C}^{k+1+\alpha}$ boundary. Let $f^{\lambda}$ and $g^{\lambda}$ be $\mathcal{C}^{\alpha}$ functions on $\partial \Omega^{\lambda}$. We consider the Dirichlet problem with parameter

$$
\Delta u^{\lambda}=0 \quad \text { on } \Omega^{\lambda}, \quad u^{\lambda}=f^{\lambda} \quad \text { on } \partial \Omega^{\lambda} .
$$

By analogy, the Neumann problem with parameter is

$$
\Delta v^{\lambda}=0 \quad \text { on } \Omega^{\lambda}, \quad \partial_{\nu^{\lambda}} v^{\lambda}=g^{\lambda} \quad \text { on } \partial \Omega^{\lambda} .
$$

Here $\Delta$ is the Laplacian and $\nu^{\lambda}$ is the outer unit normal vector of $\partial \Omega^{\lambda}$. For the existence and uniqueness of solutions $v^{\lambda}$, we impose conditions

$$
\int_{\partial \Omega^{\lambda}} g^{\lambda} d \sigma^{\lambda}=0, \quad \int_{\partial \Omega^{\lambda}} v^{\lambda} d \sigma^{\lambda}=0
$$

with $d \sigma^{\lambda}$ being the arc-length element of $\partial \Omega^{\lambda}$. We are interested in the regularity of solutions $u^{\lambda}, v^{\lambda}$ in the parameter $\lambda$. To state our results, we first define two Hölder spaces. Let integers $k, j$ satisfy $k \geq j \geq 0$. By an element $\left\{u^{\lambda}\right\}$ in $\mathcal{C}^{k+\alpha, j}(\partial \Omega)$ (resp. $\left.\mathcal{C}^{k+\alpha, j}(\bar{\Omega})\right)$ we mean a family of functions $u^{\lambda}$ on $\partial \Omega$ (resp. $\bar{\Omega}$ ) such that, for every integer $i$ with $0 \leq i \leq j, \lambda \rightarrow \partial_{\lambda}^{i} u^{\lambda}$ is a continuous map from [0,1] into $\mathcal{C}^{k-i+\alpha}(\partial \Omega)$ (resp. $\left.\mathcal{C}^{k-i+\alpha}(\bar{\Omega})\right)$. We will prove the following.

Received by the editors October 31, 2011.

2010 Mathematics Subject Classification. Primary 31A10, 45B05, 30C35, 35B30, 32H40.

Key words and phrases. Dirichlet and Neumann problems, Kellogg's theorem with parameter, domains with parameter, integral equations with parameter.

The research of the second author was supported in part by NSF grant DMS-0705426. 
Proposition 1.1. Let non-negative integers $l, k$ and $j$ satisfy $k \geq j$ and $k+1 \geq$ $l \geq j$. Let $0<\alpha<1$. Let $\Omega$ be a bounded domain in $\mathbf{C}$ with $\mathcal{C}^{k+1+\alpha}$ boundary. Let $\Gamma^{\lambda}(\lambda \in[0,1])$ embed $\bar{\Omega}$ onto $\overline{\Omega^{\lambda}}(\subset \mathbf{C})$ with $\left\{\Gamma^{\lambda}\right\}$ in $\mathcal{C}^{k+1+\alpha, j}(\bar{\Omega})$. Assume that $f^{\lambda}$ and $g^{\lambda}$ are functions on $\partial \Omega^{\lambda}$ such that $\left\{f^{\lambda} \circ \Gamma^{\lambda}\right\}$ is in $\mathcal{C}^{l+\alpha, j}(\partial \Omega)$ and $\left\{g^{\lambda} \circ \Gamma^{\lambda}\right\}$ is in $\mathcal{C}^{k+\alpha, j}(\partial \Omega)$. For each $\lambda$, let $u^{\lambda} \in \mathcal{C}^{\alpha}\left(\overline{\Omega^{\lambda}}\right)$ be the unique solution to (1.1) and let $v^{\lambda} \in \mathcal{C}^{1}\left(\overline{\Omega^{\lambda}}\right)$ be the unique solution to (1.2) $-(\underline{1.3})$. Then $\left\{u^{\lambda} \circ \Gamma^{\lambda}\right\}$ is in $\mathcal{C}^{l+\alpha, j}(\bar{\Omega})$ and $\left\{v^{\lambda} \circ \Gamma^{\lambda}\right\}$ is in $\mathcal{C}^{k+1+\alpha, j}(\bar{\Omega})$.

We observe that if a function $u$ is harmonic on the unit disc $\mathbb{D}$ and is continuous on $\overline{\mathbb{D}}$, then the product $b(\lambda) u(z)$ for a function $b$ on $[0,1]$ is still harmonic on $\mathbb{D}$. Thus, even if $b u$ is real analytic near a point $(p, 0) \in \partial \overline{\mathbb{D}} \times[0,1]$, bu might not be $\mathcal{C}^{1}$ near the same point $(p, 0) \in \overline{\mathbb{D}} \times[0,1]$. Such an example is provided when $\left.u\right|_{\partial \mathbb{D}}$ vanishes near $p$ but is not identically zero and $b$ is continuous on $[0,1]$ but not differentiable at 0 . Therefore, the regularity of solutions for the Dirichlet problem with parameter is not a local property. By contrast, the harmonic function $u$ must be $\mathcal{C}^{\omega}$ near $p \in \overline{\mathbb{D}}$ when $\left.u\right|_{\partial \mathbb{D}}$ is $\mathcal{C}^{\omega}$ near $p \in \partial \mathbb{D}$. The observation leads us to demonstrate the failure of the local Schwarz reflection principle with parameter by the following result.

Theorem 1.2. There are embeddings $\Gamma(\cdot, \lambda)$ from $\overline{\mathbb{D}}$ onto $\overline{\Omega^{\lambda}}$ such that $\Gamma$ is $C^{\infty}$ on $E=\overline{\mathbb{D}} \times[0,1]$ and real analytic at $(1,0) \in E$, but $R(\Gamma(z, \lambda), \lambda)$ is not real analytic at $(1,0) \in E$ for every family of Riemann mappings $R(\cdot, \lambda)$ from $\overline{\Omega^{\lambda}}$ onto $\overline{\mathbb{D}}$.

The existences of solutions $u^{\lambda}, v^{\lambda}$ in Proposition 1.1 are classical results; see Kel$\operatorname{logg}[8$ for the Dirichlet problem and Miranda [11] (p. 84) for work of Giraud on the Neumann problem. For a higher dimensional Dirichlet problem, see GilbargTrudinger (4], p. 211, Theorem 8.34). The reader is referred to [1] for extensive references. We will use the Fredholm theory on compact integral operators. Of course, the compactness of the integral operators is valid when the parameter is fixed and it will play important roles in our arguments, although there is no compactness when all variables are considered. With some modifications, we will follow Kellogg's approach to the Dirichlet problem (6-9]). For instance, by constructing a second resolvent, Kellogg proved the $\mathcal{C}^{1+\beta}$-regularity of the solutions to the Dirichlet problem for $\mathcal{C}^{1+\alpha}$ boundary ([7]). Instead, we will obtain the regularity of solutions to the Dirichlet problem via the integral equations associated to the Neumann problem. The reduction can be achieved because solving the Dirichlet problem on a simply connected planar domain can be reduced to finding a harmonic conjugate of the solution. We do not meet difficulties in the reduction for multi-connected domains. Using the Cauchy transform, we will also refine Kellogg's original arguments to recover a loss of regularity. We mention that Courant proved a version of Carathéodory's Riemann mapping theorem for variable Jordan domains (see 14, p. 383). Courant's theorem implies the continuous, i.e. $\mathcal{C}^{0}$, dependence of solutions to the Dirichlet problems for Jordan domains with parameter. One of the applications of solutions of the planar Dirichlet problem is Kellogg's theorem on the boundary regularity of Riemann mappings for Jordan domains of $\mathcal{C}^{1+\alpha}$ boundary 7]. Warschawski proved the sharp version of Kellogg's Riemann mapping theorem for Jordan domains of $\mathcal{C}^{k+\alpha}$ boundary for all $k>0$ ([16], [17]); see also Pommerenke [13] (p. 49). As an immediate consequence of Proposition 1.1 we get a parameter version of Kellogg's Riemann mapping theorem in Corollary 9.4 .

The paper is organized as follows. 
In section 2, we define various Hölder spaces for domains and functions with parameter. We discuss the dependence of function spaces on the parameterizations of domains and their boundaries. Section 3 contains some standard estimates on Cauchy transform (see Vekua [15]). We present details, as the arguments are used in the parameter case. In section 4, we refine Kellogg's estimates on kernels for the integral equations; lacking a reference to the sharp regularity on solutions to the integral equations, we provide some details. These arguments are generalized in section 5 for the parameter case. In section [6, after collecting results about compact operators for the Dirichlet and Neumann problems, we deduce the $\mathcal{C}^{1}$ regularity of solutions of the integral equation for the Dirichlet problem in Lemma 6.3.

Section 7 consists of our main results about the regularity of solutions of integral equations with parameter. For the proofs, we differentiate integral equations and orthogonal projections onto the null spaces of $I \pm \mathcal{K}^{\lambda}$ and $I \pm\left(\mathcal{K}^{\lambda}\right)^{*}$ and we then derive estimates by using the compactness of integral operators $\mathcal{K}^{\lambda}$ and $\left(\mathcal{K}^{\lambda}\right)^{*}$ for fixed parameter $\lambda$. In section 8 , we thoroughly discuss the Hölder spaces defined in section 2 before we define the spaces for exterior domains with parameter. In section 9. we solve the real analytic integral equations for the Dirichlet and Neumann problems with a real analytic parameter. Our main results, Theorems 9.2 and 1.2 , are proved in section 9. Proposition 1.1 is contained in Theorem 9.2 .

Note that when domains $\Omega^{\lambda}$ are fixed and only the boundary values vary with a parameter, our results essentially follow from the solutions of Dirichlet and Neumann problems without parameter. Furthermore, the results hold for general Hölder spaces with parameter (see the remark at the end of section 9). With Hölder spaces to be defined in section 2 we state the following open problem.

Problem A. Let $k, l, j$ be non-negative integers. Let $l \leq k+1$ and $0<\alpha<1$. Let $\Gamma^{\lambda}$ embed $\bar{\Omega}$ onto $\Omega^{\lambda}$, where $\Omega$ and $\Omega^{\lambda}$ are bounded domains in C. Let $u^{\lambda} \in \mathcal{C}^{0}\left(\overline{\Omega^{\lambda}}\right)$ be harmonic functions on $\Omega^{\lambda}$. Suppose that $\partial \Omega \in \mathcal{C}^{k+1+\alpha}, \Gamma \in \mathcal{C}_{*}^{k+1+\alpha, j}(\bar{\Omega})$, and $\left\{u^{\lambda} \circ \Gamma^{\lambda}\right\} \in \mathcal{C}_{*}^{l+\alpha, j}(\partial \Omega)$. Is $\left\{u^{\lambda} \circ \Gamma^{\lambda}\right\}$ in $\mathcal{C}_{*}^{l+\alpha, j}(\bar{\Omega})$ for $j>0$ ?

We would expect that, with minor modifications, analogous results for the Dirichlet and Neumann problems with parameter hold for the higher dimensional case. However, precise regularities remain to be studied. In our estimates for planar domains, we will take advantage of the Cauchy kernel in the proof of Proposition 5.2. Besides the higher dimensional Dirichlet and Neumann problems, the following problem remains open.

Problem B. Let $n \geq 2$. Let $\Omega_{1}^{\lambda}$ and $\Omega_{2}^{\lambda}$ be two $\mathcal{C}^{\infty}$ families of bounded domains in $\mathbf{C}^{n}$ with $\mathcal{C}^{\infty}$ boundaries. Assume that $\Omega_{1}^{\lambda}$ and $\Omega_{2}^{\lambda}$ are biholomorphically equivalent for each $\lambda \in[0,1]$. Does there exist a family of biholomorphic maps $f^{\lambda}$ mapping $\Omega_{1}^{\lambda}$ onto $\Omega_{2}^{\lambda}$ for $\lambda \in[0,1]$ such that $\lambda \rightarrow f^{\lambda}$ depends smoothly on $\lambda$ ?

\section{HÖLDER SPACES FOR INTERIOR DOMAINS WITH PARAMETER}

To deal with the Dirichlet problem with parameter, we will introduce two types of Hölder spaces with parameter, $\mathcal{C}^{k+\alpha, j}\left(\bar{\Omega}_{\Gamma}\right)$ and $\mathcal{B}^{k+\alpha, j}\left(\bar{\Omega}_{\Gamma}\right)$. Both are suitable for the formulation and proofs of our results. In this paper the parameter $\lambda$ will vary in $[0,1]$, unless it is restricted to a subinterval.

We first define spaces when a domain is fixed. Let $k, j$ be non-negative integers and let $0 \leq \alpha<1$. Let $\Omega$ be a bounded domain in $\mathbf{C}$. Let $\mathcal{C}^{k+\alpha}(\bar{\Omega})$ be the standard Hölder spaces with norm $|\cdot|_{k+\alpha}$ on $\bar{\Omega}$. Let $u^{\lambda}$ be a family of functions on $\bar{\Omega}$. 
We say that $\left\{u^{\lambda}\right\}$ belongs to $\mathcal{B}_{*}^{k+\alpha, j}(\bar{\Omega})$, abbreviated by $u=\left\{u^{\lambda}\right\} \in \mathcal{B}_{*}^{k+\alpha, j}(\bar{\Omega})$, if $\lambda \rightarrow \partial_{\lambda}^{i} u^{\lambda}$ maps $[0,1]$ continuously into $\mathcal{C}^{k}(\bar{\Omega})$ and boundedly into $\mathcal{C}^{k+\alpha}(\bar{\Omega})$ for each $i$ with $0 \leq i \leq j$. We say $\left\{u^{\lambda}\right\} \in \mathcal{C}_{*}^{k+\alpha, j}(\bar{\Omega})$ if $\partial_{\lambda}^{i} u^{\lambda}$ maps $[0,1]$ continuously into $\mathcal{C}^{k+\alpha}(\bar{\Omega})$ for $0 \leq i \leq j$. We define $\mathcal{B}_{*}^{k+\alpha, j}(\partial \Omega)$ and its subspace $\mathcal{C}_{*}^{k+\alpha, j}(\partial \Omega)$ by substituting $\Omega$ with $\partial \Omega \in \mathcal{C}^{k+\alpha} \cap \mathcal{C}^{1}$ in the above expressions.

Next, we define spaces on domains with parameter. Let $\Gamma^{\lambda}(0 \leq \lambda \leq 1)$ be a family of $\mathcal{C}^{1}$ embeddings from $\bar{\Omega}$ onto $\overline{\Omega^{\lambda}}$, and let $\gamma^{\lambda}(0 \leq \lambda \leq 1)$ be a family of $\mathcal{C}^{1}$ embeddings from $\partial \Omega$ onto $\partial \Omega^{\lambda}$. Suppose that $u^{\lambda}$ is a family of functions on $\overline{\Omega^{\lambda}}$ or on $\partial \Omega^{\lambda}$. Define the following:

- $\left\{u^{\lambda}\right\} \in \mathcal{B}_{*}^{k+\alpha, j}\left(\bar{\Omega}_{\Gamma}\right)$ if $\left\{u^{\lambda} \circ \Gamma^{\lambda}\right\} \in \mathcal{B}_{*}^{k+\alpha, j}(\bar{\Omega})$;

- $\left\{u^{\lambda}\right\} \in \mathcal{C}_{*}^{k+\alpha, j}\left(\bar{\Omega}_{\Gamma}\right)$ if $\left\{u^{\lambda} \circ \Gamma^{\lambda}\right\} \in \mathcal{C}_{*}^{k+\alpha, j}(\bar{\Omega})$;

- $\left\{u^{\lambda}\right\} \in \mathcal{B}_{*}^{k+\alpha, j}\left(\partial \Omega_{\gamma}\right)$ if $\left\{u^{\lambda} \circ \gamma^{\lambda}\right\} \in \mathcal{B}_{*}^{k+\alpha, j}(\partial \Omega)$;

- $\left\{u^{\lambda}\right\} \in \mathcal{C}_{*}^{k+\alpha, j}\left(\partial \Omega_{\gamma}\right)$ if $\left\{u^{\lambda} \circ \gamma^{\lambda}\right\} \in \mathcal{C}_{*}^{k+\alpha, j}(\partial \Omega)$.

For integers $k \geq j \geq 0$, define

$$
\mathcal{B}^{k+\alpha, j}\left(\bar{\Omega}_{\Gamma}\right)=\bigcap_{i=0}^{j} \mathcal{B}_{*}^{k-i+\alpha, i}\left(\bar{\Omega}_{\Gamma}\right), \quad \mathcal{C}^{k+\alpha, j}\left(\bar{\Omega}_{\Gamma}\right)=\bigcap_{i=0}^{j} \mathcal{C}_{*}^{k-i+\alpha, i}\left(\bar{\Omega}_{\Gamma}\right) .
$$

Substituting $\bar{\Omega}_{\Gamma}$ with $\partial \Omega_{\gamma}$ in the above identities, we define $\mathcal{B}^{k+\alpha, j}\left(\partial \Omega_{\gamma}\right)$ and $\mathcal{C}^{k+\alpha, j}\left(\partial \Omega_{\gamma}\right)$; dropping the subscripts $\Gamma$ and $\gamma$ from the above identities, we define $\mathcal{B}^{k+\alpha, j}(\bar{\Omega}), \mathcal{C}^{k+\alpha, j}(\bar{\Omega}), \mathcal{B}^{k+\alpha, j}(\partial \Omega)$ and $\mathcal{C}^{k+\alpha, j}(\partial \Omega)$, respectively. The norms on these spaces are defined and abbreviated as follows:

$$
\begin{gathered}
|u|_{k+\alpha, j}=\sup _{0 \leq i \leq j, \lambda \in[0,1]}\left\{\left|\partial_{\lambda}^{i} u^{\lambda}\right|_{k+\alpha}\right\} \quad \text { if } u \in \mathcal{B}_{*}^{k+\alpha, j}(\partial \Omega) \text { or } \mathcal{B}_{*}^{k+\alpha, j}(\bar{\Omega}), \\
|u|_{k+\alpha, j}=\left|\left\{u^{\lambda} \circ \Gamma^{\lambda}\right\}\right|_{k+\alpha, j}, \quad|u|_{k+\alpha, j}=\left|\left\{u^{\lambda} \circ \gamma^{\lambda}\right\}\right|_{k+\alpha, j}, \\
\|u\|_{k+\alpha, j}=\max \left\{|u|_{k-i+\alpha, i}: 0 \leq i \leq j\right\}, \quad j \leq k .
\end{gathered}
$$

The definition of spaces $\mathcal{B}_{*}^{k+\alpha, j}(\partial \Omega)$ requires $\partial \Omega \in \mathcal{C}^{k+\alpha} \cap \mathcal{C}^{1}$ implicitly. Throughout the paper, we assume that $\bar{\Omega}$ is bounded, $\partial \Omega \in \mathcal{C}^{1}, \Gamma \in \mathcal{C}^{1,0}(\bar{\Omega})$ and $\gamma \in \mathcal{C}^{1,0}(\partial \Omega)$. For $X=\partial \Omega$ or $\bar{\Omega}$ and $0 \leq j, k \leq \infty$, define $\mathcal{B}_{*}^{k+\alpha, j}(X)=\bigcap_{l<k+1, i<j+1}^{\infty} \mathcal{B}_{*}^{l+\alpha, i}(X)$. For $j \leq k \leq \infty$, define $\mathcal{B}^{k+\alpha, j}(X)=\bigcap_{i \leq l<k+1, i<j+1}^{\infty} \mathcal{B}^{l+\alpha, i}(X)$. Define analogous spaces by replacing $\mathcal{B}$ and $\mathcal{B}_{*}$ with $\mathcal{C}$ and $\mathcal{C}_{*}$, respectively.

Having defined the spaces, we now briefly discuss how they depend on the embeddings. We first need a fact to change the order of differentiation. Let $\partial_{i}=\partial_{x_{i}}$ be derivatives on $\mathbf{R}^{n}$.

Lemma 2.1. Let $f$ be a continuous function defined on an open subset $\Omega$ of $\mathbf{R}^{n}$. Assume that on $\Omega, \partial_{i_{1}} \cdots \partial_{i_{k}} f=g$ is continuous and $\partial_{i_{j_{1}}} \cdots \partial_{i_{j_{l}}} f$ are continuous for all $1 \leq j_{1}<\cdots<j_{l} \leq k$. Then $\partial_{i_{1}^{\prime}} \cdots \partial_{i_{k}^{\prime}} f$ exists and equals $g$, where $\partial_{i_{1}^{\prime}} \cdots \partial_{i_{k}^{\prime}}$ is a change of order of $\partial_{i_{1}} \cdots \partial_{i_{k}}$.

Proof. Let $\chi$ be any smooth function with compact support in $\Omega$. Replace $f$ by $\chi f$. Then $f$ satisfies the same hypotheses and it suffices to verify the assertion for the new $f$. Assume that $\operatorname{supp} f \subset(a, \infty)^{n}$ for a finite $a$. Let $X$ be the set of continuous functions on $\mathbf{R}^{n}$ with support in $(a, \infty)^{n}$. Define $\mathcal{I}_{i}: X \rightarrow X$ by

$$
\mathcal{I}_{i} \phi(x)=\int_{a}^{x_{i}} \phi\left(x_{1}, \ldots, x_{i-1}, t, x_{i+1}, \ldots, x_{n}\right) d t .
$$


Then $\mathcal{I}_{i} \mathcal{I}_{j}=\mathcal{I}_{j} \mathcal{I}_{i}$ on $X$. Also, $\partial_{i} \mathcal{I}_{i} f=f=\mathcal{I}_{i} \partial_{i} f$ if $f$ and $\partial_{i} f$ are in $X$. Now $f=\mathcal{I}_{i_{k}} \cdots \mathcal{I}_{i_{1}} g=\mathcal{I}_{i_{k}^{\prime}} \cdots \mathcal{I}_{i_{1}^{\prime}} g$, which yields $\partial_{i_{1}^{\prime}} \cdots \partial_{i_{k}^{\prime}} f=g$.

The above lemma shows that $\partial_{x, y, \lambda}^{L}=\partial_{x, y}^{I} \partial_{\lambda}^{j}$ holds on $\mathcal{C}_{*}^{k, j}(\bar{\Omega})$ if $|I|=k$ and $\partial_{x, y, \lambda}^{L}$ is obtained from $\partial_{x, y}^{I} \partial_{\lambda}^{j}$ by changing the order of differentiation. Also, $\partial_{\tau, \lambda}^{L}=\partial_{\tau}^{k} \partial_{\lambda}^{j}$ on $\mathcal{C}_{*}^{k, j}(\partial \Omega)$ if $\partial_{\tau, \lambda}^{L}$ is a change of order of $\partial_{\tau}^{k} \partial_{\lambda}^{j}$. Here $\tau$ is a continuous unit tangent vector field on $\partial \Omega$.

Lemma 2.2. Let $\bar{\Omega}$ be a bounded domain with $\partial \Omega \in \mathcal{C}^{k+\alpha} \cap \mathcal{C}^{1}$. Let $\Gamma_{1}^{\lambda}$ and $\Gamma_{2}^{\lambda}$ embed $\bar{\Omega}$ onto $\overline{\Omega^{\lambda}}$. Let $\gamma_{1}^{\lambda}$ and $\gamma_{2}^{\lambda}$ embed $\partial \Omega$ onto $\partial \Omega^{\lambda}$.

(i) $A \mathcal{C}^{1}$ mapping from $\bar{\Omega}$ into $\overline{\Omega_{1}}$ pulls back $\mathcal{C}^{\alpha, 0}\left(\overline{\Omega_{1}}\right)$ and $\mathcal{B}^{\alpha, 0}\left(\overline{\Omega_{1}}\right)$ into $\mathcal{C}^{\alpha, 0}(\bar{\Omega})$ and $\mathcal{B}^{\alpha, 0}(\bar{\Omega})$, respectively.

(ii) Let $\varphi^{\lambda}$ map $\bar{\Omega}$ into an open subset $D$ of $\mathbf{R}^{n}$. If $F$ is a function in $\mathcal{C}^{1}(D)$ and $\varphi \in \mathcal{B}^{\alpha, 0}(\bar{\Omega})$, then $\left\{F \circ \varphi^{\lambda}\right\} \in \mathcal{B}^{\alpha, 0}(\bar{\Omega})$. If $F \in \mathcal{C}^{2}(D)$ and $\varphi \in \mathcal{C}^{\alpha, 0}(\bar{\Omega})$, then $\left\{F \circ \varphi^{\lambda}\right\} \in \mathcal{C}^{\alpha, 0}(\bar{\Omega})$.

(iii) If $\Gamma_{i} \in \mathcal{B}^{k+\alpha, j}(\bar{\Omega}) \cap \mathcal{C}^{1,0}(\bar{\Omega})$, then $\mathcal{B}^{k+\alpha, j}\left(\bar{\Omega}_{\Gamma_{1}}\right)=\mathcal{B}^{k+\alpha, j}\left(\bar{\Omega}_{\Gamma_{2}}\right)$.

(iv) Let $\alpha>0$. If $\left(\Gamma_{1}^{\lambda}\right)^{-1} \Gamma_{2}^{\lambda}$ are independent of $\lambda$ and $\Gamma_{i}$ are in $\mathcal{C}^{k+\alpha, j}(\bar{\Omega}) \cap$ $\mathcal{C}^{1,0}(\bar{\Omega})$, then $\mathcal{C}^{k+\alpha, j}\left(\bar{\Omega}_{\Gamma_{1}}\right)=\mathcal{C}^{k+\alpha, j}\left(\bar{\Omega}_{\Gamma_{2}}\right)$.

The assertions in $(i)-(i v)$ remain true if $\partial \Omega, \partial \Omega_{1}, \partial \Omega^{\lambda}$, and $\gamma_{i}$ substitute for $\bar{\Omega}$, $\overline{\Omega_{1}}, \overline{\Omega^{\lambda}}$, and $\Gamma_{i}$, respectively. The identical spaces in (iii) and (iv) have equivalent norms.

Proof. (i) Since $\partial \Omega \in \mathcal{C}^{1}$, then $\left|\varphi\left(z_{2}\right)-\varphi\left(z_{1}\right)\right| \leq C\left|z_{2}-z_{1}\right|$ if $\varphi \in \mathcal{C}^{1}(\bar{\Omega})$ or $\mathcal{C}^{1}(\partial \Omega)$. The assertions follow immediately from the definition of the spaces.

(ii) We take a bounded open subset $D^{\prime}$ of $D$ such that $\overline{D^{\prime}}$ has piecewise smooth boundary and contains ranges of all $\varphi^{\lambda}$. Since $F$ is $\mathcal{C}^{1}$, then $F$ is Lipschitz on $\overline{D^{\prime}}$. It is easy to check that $\left\{F \circ \varphi^{\lambda}\right\}$ is in $\mathcal{B}^{\alpha, 0}$. Assume now that $F \in \mathcal{C}^{2}$. We already know that $\left\{F \circ \varphi^{\lambda}\right\}$ is in $\mathcal{B}^{\alpha, 0}$. Without loss of generality, we may assume that $\left|\lambda_{2}-\lambda_{1}\right|$ is so small that the range of $t \varphi^{\lambda_{2}}+(1-t) \varphi^{\lambda_{1}}$ for $0 \leq t \leq 1$ is contained in $D^{\prime}$. Then $\nabla F$ is Lipschitz on $\overline{D^{\prime}}$. Write

$$
\left(F\left(\varphi^{\lambda_{2}}\right)-F\left(\varphi^{\lambda_{1}}\right)\right)(x)=\left(\varphi^{\lambda_{2}}-\varphi^{\lambda_{1}}\right)(x) \cdot \int_{0}^{1}(\nabla F)\left(\left(t \varphi^{\lambda_{2}}+(1-t) \varphi^{\lambda_{1}}\right)(x)\right) d t .
$$

We obtain $\left|F\left(\varphi^{\lambda_{2}}\right)-F\left(\varphi^{\lambda_{1}}\right)\right|_{\alpha} \leq C\left|\varphi^{\lambda_{2}}-\varphi^{\lambda_{1}}\right|_{\alpha}\left(1+\left|\varphi^{\lambda_{2}}\right|_{\alpha}+\left|\varphi^{\lambda_{1}}\right|_{\alpha}\right)$. Hence, $\left\{F \circ \varphi^{\lambda}\right\} \in C^{\alpha, 0}$.

(iii) Let $\Gamma_{1}^{\lambda} \circ \Gamma_{12}^{\lambda}=\Gamma_{2}^{\lambda}$. Since $\Gamma_{i}^{\lambda}$ are embeddings with $\Gamma_{i} \in \mathcal{C}^{1,0}(\bar{\Omega})$, we have

$$
|\zeta-z| / C \leq\left|\Gamma_{12}^{\lambda}(\zeta)-\Gamma_{12}^{\lambda}(z)\right| \leq C|\zeta-z| .
$$

Note that on $\mathcal{C}^{k, j}(\bar{\Omega})$ all mixed derivatives of order $k-j$ in $x, y$ and of order $j$ in $\lambda$ can be written as $\partial_{x}^{k-j-a} \partial_{y}^{a} \partial_{\lambda}^{j}$. Abbreviate the latter derivatives as a set by $\partial^{k-j} \partial_{\lambda}^{j}$. For a latter purpose of expressing a commutator, it will be convenient to write first-order derivatives of a function in a column vector. So let us form the Jacobean matrix $\left(\Gamma_{i}^{\lambda}\right)^{\prime}$ of the (real) map $\Gamma_{i}^{\lambda}$ in such a way. Then the chain rule takes the form

$$
\left(\Gamma_{2}^{\lambda}\right)^{\prime}=\left(\Gamma_{12}^{\lambda}\right)^{\prime}\left(\Gamma_{1}^{\lambda}\right)^{\prime} \circ \Gamma_{12}^{\lambda}, \quad \partial_{\lambda} \Gamma_{2}^{\lambda}=\left(\partial_{\lambda} \Gamma_{1}^{\lambda}\right) \circ \Gamma_{12}^{\lambda}+\partial_{\lambda} \Gamma_{12}^{\lambda}\left(\Gamma_{1}^{\lambda}\right)^{\prime} \circ \Gamma_{12}^{\lambda} .
$$


We solve for $\partial \Gamma_{12}^{\lambda}$ and $\partial_{\lambda} \Gamma_{12}^{\lambda}$; in general, for $k \geq j$, we express $\partial^{k-j} \partial_{\lambda}^{j} \Gamma_{12}^{\lambda}$ as a polynomial in

$$
\left[\operatorname{det}\left(\Gamma_{1}^{\lambda}\right)^{\prime} \circ \Gamma_{12}^{\lambda}\right]^{-1}, \quad \partial^{a} \partial_{\lambda}^{b} \Gamma_{2}^{\lambda}, \quad\left(\partial^{a} \partial_{\lambda}^{b} \Gamma_{1}^{\lambda}\right) \circ \Gamma_{12}^{\lambda}, \quad a+b \leq k, b \leq j .
$$

To repeat the above computation for $\gamma_{i}^{\lambda}$, let $\left(\gamma_{1}^{\lambda}\right) \circ \gamma_{12}^{\lambda}=\gamma_{2}^{\lambda}$. Then

$$
\begin{gathered}
\partial_{\tau} \gamma_{2}^{\lambda}(z)=\partial_{\tau} \gamma_{12}^{\lambda}\left(\partial_{\tau} \gamma_{1}^{\lambda}\right) \circ \gamma_{12}^{\lambda}, \\
\partial_{\lambda} \gamma_{2}^{\lambda}(z)=\left(\partial_{\lambda} \gamma_{1}^{\lambda}\right) \circ \gamma_{12}^{\lambda}+\partial_{\lambda} \gamma_{12}^{\lambda}\left(\partial_{\tau} \gamma_{1}\right) \circ \gamma_{12}^{\lambda}(z) .
\end{gathered}
$$

Hence $\partial_{\tau}^{k-j} \partial_{\lambda}^{j} \gamma_{12}^{\lambda}$ is a polynomial in

$$
\left[\left(\partial_{\tau} \gamma_{1}^{\lambda}\right) \circ \gamma_{12}^{\lambda}\right]^{-1}, \quad \partial_{\tau}^{b} \partial_{\lambda}^{b} \gamma_{2}^{\lambda}, \quad\left(\partial_{\tau}^{a} \partial_{\lambda}^{b} \gamma_{1}^{\lambda}\right) \circ \gamma_{12}^{\lambda}, \quad a+b \leq k, b \leq j .
$$

Assume that $\Gamma_{1}, \Gamma_{2}$ are in $\mathcal{B}^{k+\alpha, j}$. Then functions in (2.4) are in $\mathcal{B}^{\alpha, 0}$, so $\Gamma_{12} \in$ $\mathcal{B}^{k+\alpha, j}(\bar{\Omega})$. For $u \in \mathcal{B}^{k+\alpha, j}\left(\bar{\Omega}_{\Gamma_{1}}\right)$, we express $\partial^{k-j} \partial_{\lambda}^{j}\left(u^{\lambda} \circ \Gamma_{2}^{\lambda}\right)$ as a linear combination of $\left(\partial^{a_{1}} \partial_{\lambda}^{b_{1}}\left(u^{\lambda} \circ \Gamma_{1}^{\lambda}\right)\right) \circ \Gamma_{12}^{\lambda} \in \mathcal{B}^{\alpha, 0}$ whose coefficients are polynomials in entries of (2.4). Here we replace the $(a, b)$ in (2.4) by $\left(a_{2}, b_{2}\right)$; also $a_{i}+b_{i} \leq k$ and $b_{1}+b_{2} \leq j$. Therefore, $u \in \mathcal{B}^{k+\alpha, j}\left(\bar{\Omega}_{\Gamma_{2}}\right)$. Assume now that $u \in \mathcal{B}^{k+\alpha, j}\left(\partial \Omega_{\gamma_{1}}\right)$. Then $\partial_{\tau}^{k-j} \partial_{\lambda}^{j}\left(u^{\lambda} \circ \gamma_{2}^{\lambda}\right)$ is a linear combination in $\partial_{\tau}^{a_{1}} \partial_{\lambda}^{b_{1}}\left(u^{\lambda} \circ \gamma_{2}^{\lambda}\right)$ whose coefficients are polynomials in (2.5) with $(a, b)$ being replaced by $\left(a_{2}, b_{2}\right)$. Here $a_{i}+b_{i} \leq k$ and $b_{1}+b_{2} \leq j$. Thus, we get $u \in \mathcal{B}^{k+\alpha, j}\left(\partial \Omega_{\gamma_{2}}\right)$.

(iv) Assume that $\Gamma_{1}, \Gamma_{2}$ are in $\mathcal{C}^{k+\alpha, j}(\bar{\Omega})$. By the independence of $\Gamma_{12}^{\lambda} \equiv \Gamma_{12}$ in $\lambda$ and (i), we know that all functions in (2.4) are in $\mathcal{C}^{\alpha, 0}(\bar{\Omega})$. Furthermore,

$$
\begin{aligned}
& \mid\left(\partial^{a_{1}} \partial_{\mu}^{b_{1}}\left(u^{\mu} \circ \Gamma_{1}^{\mu}\right)\right) \circ \Gamma_{12}-\left.\left(\partial^{a_{1}} \partial_{\lambda}^{b_{1}}\left(u^{\lambda} \circ \Gamma_{1}^{\lambda}\right)\right) \circ \Gamma_{12}\right|_{\alpha} \\
& \leq C\left|\partial^{a_{1}} \partial_{\mu}^{b_{1}}\left(u^{\mu} \circ \Gamma_{1}^{\mu}\right)-\partial^{a_{1}} \partial_{\lambda}^{b_{1}}\left(u^{\lambda} \circ \Gamma_{1}^{\lambda}\right)\right|_{\alpha} .
\end{aligned}
$$

Let $u \in \mathcal{C}^{k+\alpha, j}\left(\bar{\Omega}_{\Gamma_{1}}\right)$. The above inequality shows that $\left(\partial^{a_{1}} \partial_{\lambda}^{b_{1}}\left(u^{\lambda} \circ \Gamma_{1}^{\lambda}\right)\right) \circ \Gamma_{12}^{\lambda}$ are in $\mathcal{C}^{\alpha, 0}(\bar{\Omega})$. By (ii), the latter is closed under addition, multiplication, and division (for the non-vanishing denominator); hence, $u$ is in $\mathcal{C}^{k+\alpha, j}\left(\bar{\Omega}_{\Gamma_{2}}\right)$. By analogy, we can verify that $\mathcal{C}^{k+\alpha, j}\left(\partial \Omega_{\gamma_{1}}\right)=\mathcal{C}^{k+\alpha, j}\left(\partial \Omega_{\gamma_{2}}\right)$. For (iii) and (iv), the equivalence of norms is easy to verify too.

We now set up some notation to be used throughout the paper.

We assume that $\Omega$ and $\Omega^{\lambda}$ are bounded domains of at least $\mathcal{C}^{1}$ boundary. We denote by $\hat{\gamma}_{0}$ the outer boundary of $\Omega$ and by $\hat{\gamma}_{1}, \ldots, \hat{\gamma}_{m}$ the connected components of its inner boundary. Without loss of generality, we choose the standard orientation for $\partial \Omega$ and $\partial \Omega^{\lambda}$ and assume that $\mathcal{C}^{1}$ embeddings $\gamma^{\lambda}: \partial \Omega \rightarrow \partial \Omega^{\lambda}$ preserve the orientation and send outer boundary to outer boundary. Denote by $\tau$ and $\tau^{\lambda}$ the unit tangential vectors of $\partial \Omega$ and $\partial \Omega^{\lambda}$ that agree with the orientation, and by $\nu$ and $\nu^{\lambda}$ the outer unit normal vectors of $\partial \Omega$ and $\partial \Omega^{\lambda}$. The arc-length elements on $\partial \Omega$ and $\partial \Omega^{\lambda}$ are denoted by $d \sigma$ and $d \sigma^{\lambda}$, respectively. Sometimes we parameterize $\partial \Omega$ by $\hat{\gamma}(t)$ in arc-length such that $d t$ agrees with the orientation of $\partial \Omega$, and we regard $\tau_{z}$ and $\hat{\gamma}^{\prime}(t)$ as complex numbers instead of vectors. With the above notation, on $\partial \Omega$ we have

$$
d f=\partial_{\tau} f d \sigma, \quad d \sigma(\zeta)=\bar{\tau}_{\zeta} d \zeta, \quad d \sigma^{\lambda}=\left|\partial_{\tau} \gamma^{\lambda}\right| d \sigma .
$$

To simplify the use of the chain rule, we need to compute derivatives in $\partial \Omega^{\lambda}$ or $\overline{\Omega^{\lambda}}$. At $z^{\lambda}=\gamma^{\lambda}(z)$, we define $\left(\partial_{\lambda} u^{\lambda}\right)\left(z^{\lambda}\right)=\partial_{\lambda}\left(u^{\lambda}\left(\gamma^{\lambda}\right)\right)$ and

$$
\tau_{z}^{\lambda}=\left|\partial_{\tau} \gamma^{\lambda}\right|^{-1} \partial_{\tau} \gamma^{\lambda}(z), \quad\left(\partial_{\tau^{\lambda}} u^{\lambda}\right)\left(z^{\lambda}\right)=\left|\partial_{\tau} \gamma^{\lambda}(z)\right|^{-1} \partial_{\tau}\left(u^{\lambda}\left(\gamma^{\lambda}\right)\right)(z) .
$$


Note that $\left[\partial_{\lambda}, \partial_{\tau}\right]=0$ on $\mathcal{C}_{*}^{1,1}(\partial \Omega)$. On $\mathcal{C}_{*}^{1,1}\left(\partial \Omega_{\gamma}\right)$ with $\gamma \in \mathcal{C}_{*}^{1,1}(\partial \Omega)$ we define and compute the following commutator:

$$
\begin{gathered}
{\left[\partial_{\lambda}, \partial_{\tau_{z}^{\lambda}}\right]\left(f^{\lambda}\left(z^{\lambda}\right)\right)=\partial_{\lambda}\left[\partial_{\tau_{z}^{\lambda}}\left(f^{\lambda}\left(z^{\lambda}\right)\right)\right]-\partial_{\tau_{z}^{\lambda}}\left[\partial_{\lambda}\left(\left(f^{\lambda}\left(z^{\lambda}\right)\right)\right)\right]} \\
{\left[\partial_{\lambda}, \partial_{\tau_{z}^{\lambda}}\right]=\left|\partial_{\tau_{z}} \gamma^{\lambda}\right| \partial_{\lambda}\left|\partial_{\tau_{z}} \gamma^{\lambda}\right|^{-1} \partial_{\tau_{z}^{\lambda}}=-\left(\partial_{\lambda} \log \left|\partial_{\tau_{z}} \gamma^{\lambda}\right|\right) \partial_{\tau_{z}^{\lambda}} .}
\end{gathered}
$$

Therefore, for $\gamma \in \mathcal{C}_{*}^{i, j}(\partial \Omega) \cap \mathcal{C}_{*}^{1,0}(\partial \Omega)$, we have

$$
\begin{gathered}
\partial_{\tau^{\lambda}}: \mathcal{C}_{*}^{i, j}\left(\partial \Omega_{\gamma}\right) \rightarrow \mathcal{C}_{*}^{i-1, j}\left(\partial \Omega_{\gamma}\right), \quad \partial_{\lambda}: \mathcal{C}_{*}^{i, j}\left(\partial \Omega_{\gamma}\right) \rightarrow \mathcal{C}_{*}^{i, j-1}\left(\partial \Omega_{\gamma}\right), \\
{\left[\partial_{\lambda}, \partial_{\tau^{\lambda}}\right]: \mathcal{C}_{*}^{i, j}\left(\partial \Omega_{\gamma}\right) \rightarrow \mathcal{C}_{*}^{i-1, j-1}\left(\partial \Omega_{\gamma}\right)}
\end{gathered}
$$

whenever the exponents are non-negative. For $\gamma \in \mathcal{C}^{k, j}(\partial \Omega)$, we have

$$
\begin{gathered}
\partial_{\tau^{\lambda}}: \mathcal{C}^{k, j}\left(\partial \Omega_{\gamma}\right) \rightarrow \mathcal{C}^{k-1, j}\left(\partial \Omega_{\gamma}\right), \quad k-1 \geq j \\
\partial_{\lambda}: \mathcal{C}^{k, j}\left(\partial \Omega_{\gamma}\right) \rightarrow \mathcal{C}^{k-1, j-1}\left(\partial \Omega_{\gamma}\right) \\
{\left[\partial_{\lambda}, \partial_{\tau^{\lambda}}\right]: \mathcal{C}^{k, j}\left(\partial \Omega_{\gamma}\right) \rightarrow \mathcal{C}^{k-2, j-1}\left(\partial \Omega_{\gamma}\right), \quad k \geq j+1 \geq 2 ;} \\
{\left[\partial_{\lambda}, \partial_{\tau^{\lambda}}\right]: \mathcal{C}^{k, j}\left(\partial \Omega_{\gamma}\right) \rightarrow \mathcal{C}^{k-1, j-1}\left(\partial \Omega_{\gamma}\right), \quad \text { for } \gamma \in \mathcal{C}^{k+1, j}(\partial \Omega) \text { and } k \geq 1 .}
\end{gathered}
$$

Throughout the paper, we denote by $C_{k+\alpha, j}$ or $C$ a constant which depends on

$$
\sup _{\lambda}\left|\operatorname{det}\left(\Gamma^{\lambda}\right)^{\prime}\right|_{0}^{-1},\left|\left(\Gamma^{\lambda}\right)^{\prime}\right|_{0},\|\Gamma\|_{k+\alpha, j}, \|\left.\left.\hat{\gamma}^{\prime}\right|^{-1}\right|_{0},\left|\hat{\gamma}^{\prime}\right|_{0},|\hat{\gamma}|_{k+\alpha}
$$

where $\hat{\gamma}$ is a parameterization for $\partial \Omega$ of class $\mathcal{C}^{k+\alpha} \cap \mathcal{C}^{1}$. We also denote by $C_{k+\alpha}$ or $C$ a constant which depends on the last three quantities. The constants $\mathcal{C}_{l+\beta, j}^{*}$ will depend only on quantities in (2.10), where $\|\Gamma\|_{k+\alpha, j}$ is replaced by $|\Gamma|_{l+\alpha, j}$.

A consequence of (2.7)-(2.9) is the following.

Lemma 2.3. Let $\partial \Omega \in \mathcal{C}^{k+\alpha} \cap \mathcal{C}^{1}$. Let $\gamma^{\lambda}$ embed $\partial \Omega$ onto $\partial \Omega^{\lambda}$ with $\gamma \in \mathcal{B}^{k+\alpha, j}(\partial \Omega)$ $\cap \mathcal{C}^{1,0}(\partial \Omega)$. Then $\left\{u^{\lambda}\right\} \in \mathcal{B}^{k+\alpha, j}\left(\partial \Omega_{\gamma}\right)$ if and only if $\left\{\partial_{\tau^{\lambda}}^{a} \partial_{\lambda}^{b} u^{\lambda}\right\}$ or $\left\{\partial_{\lambda}^{b} \partial_{\tau^{\lambda}}^{a} u^{\lambda}\right\}$ is in $\mathcal{B}^{\alpha, 0}\left(\partial \Omega_{\gamma}\right)$ for every $(a, b)$ with $a+b \leq k$ and $b \leq j$. Moreover,

$$
C_{k+\alpha, j}^{-1}\|u\|_{k+\alpha, j} \leq \sum_{a+b \leq k, b \leq j} \sup _{\lambda}\left\|\partial_{\tau^{\lambda}}^{a} \partial_{\lambda}^{b} u^{\lambda}\right\|_{\alpha, 0} \leq C_{k+\alpha, j}\|u\|_{k+\alpha, j}
$$

These conclusions remain true if $\mathcal{C}^{k+\alpha, j}$ and $\mathcal{C}^{\alpha, 0}$ substitute for $\mathcal{B}^{k+\alpha, j}$ and $\mathcal{B}^{\alpha, 0}$, respectively.

We distinguish the first-order derivatives on $\Omega^{\lambda}$ by $\partial_{x^{\lambda}}$ in real variables $x^{\lambda}$ and denote the first-order derivatives on $\Omega$ by $\partial_{x}$. Then for $x^{\lambda}=\Gamma^{\lambda}(x)$,

$$
\partial_{x^{\lambda}} u^{\lambda}=\left(\partial_{x} \Gamma^{\lambda}\right)^{-1} \partial_{x}\left(u^{\lambda} \circ \Gamma^{\lambda}\right) .
$$

Combining with $\left[\partial_{\lambda}, \partial_{x}\right]=0$, we define and compute on $\mathcal{C}_{*}^{1,1}\left(\bar{\Omega}_{\Gamma}\right)$ with $\Gamma \in \mathcal{C}_{*}^{1,1}(\bar{\Omega})$ the following commutator:

$$
\begin{gathered}
{\left[\partial_{\lambda}, \partial_{x^{\lambda}}\right]\left(f^{\lambda}\left(x^{\lambda}\right)\right)=\partial_{\lambda}\left[\partial_{x^{\lambda}}\left(f^{\lambda}\left(x^{\lambda}\right)\right)\right]-\partial_{x^{\lambda}}\left[\partial_{\lambda}\left(\left(f^{\lambda}\left(x^{\lambda}\right)\right)\right)\right]} \\
{\left[\partial_{\lambda}, \partial_{x^{\lambda}}\right]=\partial_{\lambda}\left(\left(\partial_{x} \Gamma^{\lambda}\right)^{-1}\right) \partial_{x} \Gamma^{\lambda} \partial_{x^{\lambda}} .}
\end{gathered}
$$


We denote by $\partial_{x^{\lambda}}^{a}$ the derivatives of order $a$ in $x^{\lambda}$. The following can be easily verified.

Lemma 2.4. Let $\Gamma^{\lambda}$ embed $\bar{\Omega}$ onto $\overline{\Omega^{\lambda}}$ with $\Gamma \in \mathcal{B}^{k+\alpha, j}(\bar{\Omega}) \cap \mathcal{C}^{1,0}(\bar{\Omega})$. Then $\left\{u^{\lambda}\right\} \in$ $\mathcal{B}^{k+\alpha, j}\left(\bar{\Omega}_{\Gamma}\right)$ if and only if $\left\{\partial_{x^{\lambda}}^{a} \partial_{\lambda}^{b} u^{\lambda}\right\}$ or $\left\{\partial_{\lambda}^{b} \partial_{x^{\lambda}}^{a} u^{\lambda}\right\}$ is in $\mathcal{B}^{\alpha, 0}\left(\bar{\Omega}_{\Gamma}\right)$ for every $(a, b)$ with $a+b \leq k$ and $b \leq j$. Moreover,

$$
C_{k+\alpha, j}^{-1}\|u\|_{k+\alpha, j} \leq \sum_{a+b \leq k, b \leq j}\left\|\partial_{x^{\lambda}}^{a} \partial_{\lambda}^{b} u^{\lambda}\right\|_{\alpha, 0} \leq C_{k+\alpha, j}\|u\|_{k+\alpha, j} .
$$

The conclusions remain true if $\mathcal{C}^{k+\alpha, j}$ and $\mathcal{C}^{\alpha, 0}$ substitute for $\mathcal{B}^{k+\alpha, j}$ and $\mathcal{B}^{\alpha, 0}$, respectively.

We have seen the dependence of spaces $\mathcal{C}^{k+\alpha, j}$ in parameterizations through Lemma 2.2. Throughout the paper, we assume that $\gamma^{\lambda}$ is the restriction of $\Gamma^{\lambda}$ on $\partial \Omega$. In section 8 we will return to further discuss the spaces $\mathcal{C}^{k+1+\alpha, j}$ and $\mathcal{B}^{k+1+\alpha, j}$ and define Hölder spaces for exterior domains.

We conclude the section with further notation. Recall that $\Omega \in \mathcal{C}^{1}$ is bounded and has the standard orientation. On $\partial \Omega \times \partial \Omega$ and off its diagonal, define $K(z, \zeta)=$ $\frac{1}{\pi} \partial_{\tau_{\zeta}} \arg (z-\zeta)$. By (2.6), we have $K(z, \zeta) d \sigma(\zeta)=\frac{1}{\pi} d_{\zeta} \arg (z-\zeta)$, and hence

$$
\int_{\partial \Omega} K(z, \zeta) d \sigma(\zeta)=1, \quad z \in \partial \Omega
$$

A basic property of kernel $K$ is that $|K(z, \zeta)| \leq C|\zeta-z|^{\alpha-1}$ for $\zeta, z \in \partial \Omega$ when $\partial \Omega \in \mathcal{C}^{1+\alpha}$ with $0<\alpha<1$. By Fubini's theorem and Hölder inequalities (or Young's inequality), we have two bounded operators on $L^{p}(\partial \Omega)(p \geq 1)$ :

$$
\mathcal{K} f(z)=\int_{\partial \Omega} f(\zeta) K(z, \zeta) d \sigma(\zeta), \quad \mathcal{K}^{*} f(z)=\int_{\partial \Omega} f(\zeta) K(\zeta, z) d \sigma(\zeta)
$$

These two operators play important roles in solving the Dirichlet and Neumann problems. We will regard $\mathcal{K}$ and $\mathcal{K}^{*}$ as operators on $L^{1}(\partial \Omega)$, unless otherwise specified.

\section{Integral equations for Dirichlet and Neumann Problems}

In this section and the next, we will present some standard estimates about integral operators. For the sake of exposition clarity, we include some details for the estimates. We will then adapt these estimates in section 5 for the parameter case.

Let $\Omega$ be a bounded domain in $\mathbf{C}$ with $\mathcal{C}^{1}$ boundary and let $f \in L^{1}(\partial \Omega)$. On $\Omega$ and $\Omega^{\prime}=\mathbf{C} \backslash \bar{\Omega}$, the double and single potentials with moment $f$ are respectively

$$
\begin{gathered}
U f(z)=\frac{1}{\pi} \int_{\partial \Omega} f(\zeta) \partial_{\tau_{\zeta}} \arg (z-\zeta) d \sigma(\zeta), \\
W f(z)=\frac{1}{\pi} \int_{\partial \Omega} f(\zeta) \log |z-\zeta| d \sigma(\zeta) .
\end{gathered}
$$

The following formulae lead the classical solutions of the Dirichlet and Neumann problems via the Fredholm theory; for instance, see [10] (pp. 381-390). 
Proposition 3.1. Let $\partial \Omega \in \mathcal{C}^{1+\alpha}$ with $0<\alpha<1$. Suppose that $f$ is a continuous function on $\partial \Omega$. Then $W f$ is continuous on $\mathbf{C}$ and $U f$ extends to functions $U^{+} f \in$ $\mathcal{C}^{0}(\bar{\Omega})$ and $U^{-} f \in \mathcal{C}^{0}\left(\overline{\Omega^{\prime}}\right)$. On $\partial \Omega$,

$$
\begin{gathered}
U^{+} f=f+\mathcal{K} f, \quad U^{-} f=-f+\mathcal{K} f ; \\
\partial_{\nu} W f=f+\mathcal{K}^{*} f, \quad-\partial_{-\nu} W f=-f+\mathcal{K}^{*} f .
\end{gathered}
$$

Proof. Recall that we parameterize $\partial \Omega$ by $\gamma(t)$ such that $d t$ is the arc-length element agreeing with the standard orientation of $\partial \Omega$. Let $l$ be the arc-length of $\partial \Omega$. We abbreviate $f(\gamma(t)), \tau(\gamma(t))$, and $\nu(\gamma(t))$ by $f(t), \tau(t)$, and $\nu(t)$, respectively.

Write $\gamma(t)=\gamma(s)+\tau(s)(t-s)+R(t, s)$ with $|R(t, s)| \leq|t-s| / 4$ for $|t-s|<1 / C$. Then

$$
\sqrt{h^{2}+|t-s|^{2}} / 2 \leq|\gamma(s)+h \nu(s)-\gamma(t)| \leq 2 \sqrt{h^{2}+|t-s|^{2}} .
$$

For a latter purpose we remark that the above merely needs $\gamma \in \mathcal{C}^{1}$. Note that $\nu(t) \cdot(\gamma(t)-\gamma(s))=\nu(t) \cdot \int_{s}^{t}\left(\gamma^{\prime}(r)-\gamma^{\prime}(t)\right) d r$. Returning to the condition $\gamma \in \mathcal{C}^{1+\alpha}$, we have, for $|t-s|<1 / C$,

$$
\frac{|\nu(t) \cdot(\gamma(t)-\gamma(s))|}{|\gamma(s)+h \nu(s)-\gamma(t)|^{2}} \leq \frac{C|s-t|^{1+\alpha}}{|t-s|^{2}+h^{2}} \leq C|s-t|^{\alpha-1} .
$$

In particular,

$$
k(s, t) \stackrel{\text { def }}{=} \partial_{t} \arg (\gamma(s)-\gamma(t))=\frac{\nu(t) \cdot(\gamma(t)-\gamma(s))}{|\gamma(s)-\gamma(t)|^{2}}
$$

satisfies $|k(s, t)| \leq C|s-t|^{\alpha-1}$, and $k(s, \cdot)$ is integrable.

Recall that

$$
U f(z)=\frac{1}{\pi} \int_{0}^{l} f(t) \partial_{t} \arg (z-\gamma(t)) d t .
$$

Fix a small $\epsilon>0$ and $\gamma(s) \in \partial \Omega$. Let $\delta=\operatorname{dist}(z, \partial \Omega)$. Choose $s_{*}$ such that $\left|\gamma\left(s_{*}\right)-z\right|=\delta$. Note that as $\gamma \in \mathcal{C}^{1+\alpha}$ with $\alpha<1, s_{*}$ may not be unique even if $\delta$ is sufficiently small. Nevertheless, $z=\gamma\left(s_{*}\right)+\delta \nu\left(s_{*}\right)$. Let $|z-\gamma(s)|$ be so small that $\left|s_{*}-s\right|<\epsilon / 2$. We have

$$
\partial_{t} \arg (z-\gamma(t))=\frac{\nu(t) \cdot\left(\gamma(t)-\gamma\left(s_{*}\right)\right)}{|\gamma(t)-z|^{2}}+\frac{\nu(t) \cdot\left(\gamma\left(s_{*}\right)-z\right)}{|\gamma(t)-z|^{2}} .
$$

By (3.5)-(3.6), we get

$$
\left|\partial_{t} \arg (z-\gamma(t))\right|=\frac{|\nu(t) \cdot(\gamma(t)-z)|}{|\gamma(t)-z|^{2}} \leq C \frac{\left|t-s_{*}\right|^{1+\alpha}+\delta}{\delta^{2}+\left|t-s_{*}\right|^{2}} .
$$

Since $s_{*}$ depends only on $z$, this shows that

$$
\int_{0}^{l}\left|\partial_{t} \arg (z-\gamma(t))\right| d t<C_{0}, \quad z \in \mathbf{C} .
$$

Here $C_{0}$ is independent of $s_{*}, z$ and $\delta$. We have

$$
\begin{aligned}
& \left|\int_{0}^{l}(f(t)-f(s))\left(\partial_{t} \arg (z-\gamma(t))-\partial_{t} \arg (\gamma(s)-\gamma(t))\right) d t\right| \\
& \leq 2\|f\|_{0} \sup _{|\zeta-\gamma(s)|>\epsilon}\left|\partial_{t} \arg (z-\gamma(t))-\partial_{t} \arg (\gamma(s)-\gamma(t))\right| \\
& \quad+2 C_{0} \sup _{|t-s|<\epsilon}|f(t)-f(s)| .
\end{aligned}
$$


By (3.7) and the continuity of $f$ at $s$, we conclude

$$
\begin{aligned}
\lim _{\partial \Omega \not \supset z \rightarrow \gamma(s)} & \int_{0}^{l}(f(t)-f(s)) \partial_{t} \arg (z-\gamma(t)) d t \\
= & \int_{0}^{l}(f(t)-f(s)) \partial_{t} \arg (\gamma(s)-\gamma(t)) d t .
\end{aligned}
$$

Expand both sides. By the values of $\int_{0}^{l} \partial_{t} \arg (z-\gamma(t)) d t$ on $\mathbf{C}$, we get (3.3).

For (3.4), recall that

$$
W f(z)=\frac{1}{\pi} \int_{0}^{l} f(t) \log |z-\gamma(t)| d t
$$

We want to show that the interior and exterior normal derivatives of $W f$ exist at $\gamma(s)$. Let $|h|>0$ be small. By the fundamental theorem of calculus, we have

$$
\begin{array}{r}
\frac{W f(\gamma(s)+h \nu(s))-W f(\gamma(s))}{h / \pi}=\int_{0}^{l} \int_{0}^{1} f(t) \frac{\nu(s) \cdot(\gamma(s)-\gamma(t)) d r d t}{|\gamma(s)+r h \nu(s)-\gamma(t)|^{2}} \\
+\int_{0}^{l} \int_{0}^{1} \frac{f(t) r h d r d t}{|\gamma(s)+r h \nu(s)-\gamma(t)|^{2}} \stackrel{\text { def }}{=} R_{1}(s, h)+R_{2}(s, h) .
\end{array}
$$

We see that $R_{1}(s, h)$ tends to $\int_{0}^{l} f(t) \frac{\nu(s) \cdot(\gamma(s)-\gamma(t))}{|\gamma(s)-\gamma(t)|^{2}} d t$ as $h \rightarrow 0$, by (3.6) and the dominated convergence theorem.

Decompose $R_{2}$ into integrals $R_{\epsilon}^{\prime}, R_{\epsilon}^{\prime \prime}$ in $(t, r)$ with $|t-s|<\epsilon$ and $|t-s|>\epsilon$, respectively. It is immediate that, for fixed $\epsilon>0, R_{\epsilon}^{\prime \prime}(s, h)$ tends to 0 as $h \rightarrow 0$. Note that the integrand in $R_{2}$ does not change the sign when $f \geq 0$. By the continuity of $f$, it remains to show that when $f \equiv 1$,

$$
\lim _{\epsilon \rightarrow 0} \lim _{h \rightarrow 0^{+}} R_{\epsilon}^{\prime}(s, h)=\pi, \quad \lim _{\epsilon \rightarrow 0} \lim _{h \rightarrow 0^{-}} R_{\epsilon}^{\prime}(s, h)=-\pi .
$$

Let $E(s, t)=\gamma(s)-\gamma(t)+\gamma^{\prime}(s)(t-s)$. Then $|E(s, t)| \leq C|s-t|^{1+\alpha}$, and for $|h|<1$,

$$
\begin{aligned}
\begin{aligned}
|\gamma(s)+h \nu(s)-\gamma(t)|^{2} & =|-\tau(s)(t-s)+h \nu(s)+E(s, t)|^{2} \\
& =(s-t)^{2}+h^{2}+\tilde{E}(s, t, h),
\end{aligned} \\
|\tilde{E}(s, t, h)| \leq C\left(|h||t-s|^{1+\alpha}+|t-s|^{2+\alpha}\right) \leq 2 C \epsilon^{\alpha}\left(h^{2}+|s-t|^{2}\right) .
\end{aligned}
$$

Let $h$ tend to $0^{+}$and then let $\epsilon$ tend to $0^{+}$. We get

$$
R_{\epsilon}^{\prime}(s, h)=\left(1+C \epsilon^{\alpha}\right) \int_{|t-s|<\epsilon} \int_{0}^{1} \frac{r h d r d t}{(s-t)^{2}+(r h)^{2}} \rightarrow \pi .
$$

This yields the first identity in (3.9). The second is obtained by analogy.

Let $\Omega$ be a bounded domain in $\mathbf{C}$ with $\mathcal{C}^{1}$ boundary. Recall the Cauchy transform

$$
\mathcal{C} f(z)=\frac{1}{2 \pi i} \int_{\partial \Omega} \frac{f(\zeta)}{\zeta-z} d \zeta
$$

on $\mathbf{C} \backslash \partial \Omega$ for $f \in L^{1}(\partial \Omega)$. Away from $\partial \Omega$,

$$
U f=2 \operatorname{Re} \mathcal{C} f, \quad \text { for } f=\bar{f} ; \quad \partial_{z} W f=-i \mathcal{C}[\bar{\tau} f] .
$$

We will derive estimates of $U f, W f$ via $\mathcal{C} f$ when $f$ is in Hölder spaces.

Lemma 3.2. Let $0<\alpha<1$ and let $k, l \geq 0$ be integers. Let $\Omega$ be a bounded domain in $\mathbf{C}$ with $\partial \Omega \in \mathcal{C}^{1}$ and let $\Omega^{\prime}=\mathbf{C} \backslash \bar{\Omega}$. 
(i) Let $f$ be a function in $C^{\alpha}(\partial \Omega)$. Then $\mathcal{C} f$ extends to functions $\mathcal{C}^{+} f \in \mathcal{C}^{\alpha}(\bar{\Omega})$ and $\mathcal{C}^{-} f \in \mathcal{C}^{\alpha}\left(\overline{\Omega^{\prime}}\right)$. Moreover, on $\partial \Omega$,

$$
\mathcal{C}^{-} f(z)=\frac{1}{2 \pi i} \int_{\partial \Omega} \frac{f(z)-f(\zeta)}{z-\zeta} d \zeta, \quad \mathcal{C}^{+} f(z)-\mathcal{C}^{-} f(z)=f(z) .
$$

(ii) Let $f \in \mathcal{C}^{l+\alpha}(\partial \Omega)$ and $\partial \Omega \in \mathcal{C}^{k+1+\alpha}$ with $k+1 \geq l$. Then $\mathcal{C}^{+} f \in \mathcal{C}^{l+\alpha}(\bar{\Omega})$ and $\mathcal{C}^{-} f \in \mathcal{C}^{l+\alpha}\left(\overline{\Omega^{\prime}}\right)$. If $f$ and $\partial \Omega$ are real analytic, then $\mathcal{C}^{+} f \in \mathcal{C}^{\omega}(\bar{\Omega})$.

(iii) If $f \in L^{\infty}(\partial \Omega)$, then $W f$ extends to a continuous function on $\mathbf{C}$.

Proof. (i) Let $z \notin \partial \Omega$ and let $z_{*}=\gamma(s)$ satisfy $\left|z-z_{*}\right|=\operatorname{dist}(z, \partial \Omega)=\delta$. Assume that $\delta$ is small. We have

$$
(\mathcal{C} f)^{\prime}(z)=\frac{1}{2 \pi i} \int_{\partial \Omega} \frac{f(\zeta)-f\left(z_{*}\right)}{(\zeta-z)^{2}} d \zeta .
$$

By (3.5),$\left|(\mathcal{C} f)^{\prime}(z)\right| \leq C \int_{0}^{\infty}(r+\delta)^{\alpha-2} d r \leq C^{\prime} \delta^{-1+\alpha}=C^{\prime} \operatorname{dist}(z, \partial \Omega)^{-1+\alpha}$. By the Hardy-Littlewood lemma, $\mathcal{C} f$ is of class $\mathcal{C}^{\alpha}$ on $\bar{\Omega}$ and $\overline{\Omega^{\prime}}$.

To find the boundary values of $\mathcal{C}^{+} f$ and $\mathcal{C}^{-} f$, it suffices to compute limits of $\mathcal{C} f$ in the normal directions. Let $z=\gamma(s)+\delta \nu(s) \in \Omega^{\prime}$ and $z_{*}=\gamma(s)$. Write

By (3.5), we obtain

$$
\mathcal{C}^{-} f(z)=\frac{1}{2 \pi i} \int_{\partial \Omega} \frac{f(\zeta)-f\left(z_{*}\right)}{\zeta-z} d \zeta
$$

$$
\frac{|f(\gamma(t))-f(\gamma(s))|}{|\gamma(t)-z|} \leq C|t-s|^{\alpha-1}
$$

By the dominated convergence theorem, we find on $\partial \Omega$

$$
\mathcal{C}^{-} f\left(z_{*}\right)=\frac{1}{2 \pi i} \int_{\partial \Omega} \frac{f(\zeta)-f\left(z_{*}\right)}{\zeta-z_{*}} d \zeta
$$

Analogously, we can verify the formula for $\mathcal{C}^{+} f$.

(ii) For higher-order derivatives, for $l \leq k+1$ we get from (2.6)

$$
\begin{gathered}
\partial_{z} \mathcal{C} f(z)=\frac{1}{2 \pi i} \int_{\partial \Omega} \frac{f(\zeta) d \zeta}{(\zeta-z)^{2}}=\frac{1}{2 \pi i} \int_{\partial \Omega} \bar{\tau} \partial_{\tau} f(\zeta) \frac{d \zeta}{\zeta-z} \\
\partial_{z}^{l} \mathcal{C} f(z)=\frac{1}{2 \pi i} \int_{\partial \Omega} \frac{\left(\bar{\tau} \partial_{\tau}\right)^{l} f(\zeta)}{\zeta-z} d \zeta \\
\partial_{z}^{l+1} \mathcal{C} f(z)=\frac{1}{2 \pi i} \int_{\partial \Omega} \frac{\left(\bar{\tau} \partial_{\tau}\right)^{l} f(\zeta)-\left(\bar{\tau} \partial_{\tau}\right)^{l} f\left(z_{*}\right)}{(\zeta-z)^{2}} d \zeta
\end{gathered}
$$

By (3.5) again, we obtain

$$
\begin{gathered}
\left|\partial_{z}^{l} \mathcal{C} f(z)\right| \leq\left|\left(\bar{\tau} \partial_{\tau}\right)^{l} f\right|_{0}+C_{1}\left|\left(\bar{\tau} \partial_{\tau}\right)^{l} f\right|_{\alpha}, \\
\left|\partial_{z}^{l+1} \mathcal{C} f(z)\right| \leq C_{1}\left|\left(\bar{\tau} \partial_{\tau}\right)^{l} f\right|_{\alpha} \operatorname{dist}(z, \partial \Omega)^{-1+\alpha} .
\end{gathered}
$$

Therefore, $\mathcal{C} f$ is of class $\mathcal{C}^{l+\alpha}$ on $\bar{\Omega}$ and $\overline{\Omega^{\prime}}$.

For the real analytic case, we note that the constant $C_{1}$ in (3.13) is independent of $l$. By Taylor's theorem, a function $f$ on $\bar{\Omega}$ with $\partial \Omega \in \mathcal{C}^{\omega}$ is real analytic if and only if

$$
\left|\partial_{\bar{z}}^{i} \partial_{z}^{j} f(z)\right| \leq i ! j ! C^{i+j+1}
$$

for some $C>1$ independent of $z$. Note that $\left|\left(\bar{\tau} \partial_{\tau}\right)^{l} f\right|_{\alpha} \leq C\left|\left(\bar{\tau} \partial_{\tau}\right)^{l+1} f(z)\right|_{0}$. By (3.13) it suffices to show that near each point $z_{0} \in \partial \Omega$, we have

$$
\left|\left(\bar{\tau} \partial_{\tau}\right)^{l} f(z)\right| \leq C^{l+1} l ! .
$$


Let $x \rightarrow \varphi(x)$ be a local real analytic parameterization of $\partial \Omega$ with $\varphi(0)=z_{0}$. Then $\left(d \varphi^{-1}\right)\left(\bar{\tau} \partial_{\tau}\right)$ is given by $A(x) \partial_{x}$ with $A \neq 0$. Extend $\varphi(x), A(x)$ and $f(\varphi(x))$ as holomorphic functions and denote them by the same symbols. We find local holomorphic coordinates $z=\psi(w)$ such that $\left(d_{w} \psi\right)^{-1}\left(A \partial_{z}\right)=\partial_{w}$. Then $\left(\bar{\tau} \partial_{\tau}\right)^{l} f(\zeta)=\partial_{w}^{l}(f \circ \varphi \circ \psi)(w)$ with $\zeta=\varphi \circ \psi(w)$. Since $f \circ \varphi \circ \psi$ is holomorphic, we easily get (3.14).

(iii) One can verify the continuity of $W f$ via (3.5).

\section{Derivatives of $\mathcal{K} f$ and $\mathcal{K}^{*} f$}

In this section, we recall some calculations on kernels by Kellogg [7, 8] and express $\mathcal{K}$ and $\mathcal{K}^{*}$ via the Cauchy transform. We write $\nu_{\gamma(t)}=\nu(t), \tau_{\gamma(t)}=\tau(t)$ and $f(\gamma(t))=f(t)$. Let $l_{j}$ be the length of the $j$-th component $\gamma_{j}$ of $\partial \Omega$. Recall that $\gamma_{0}$ is the outer boundary of $\partial \Omega$. Set $l_{-1}=0$.

Lemma 4.1. Let $\partial \Omega \in \mathcal{C}^{k+1+\alpha}$ with $k \geq 0$ and $0<\alpha<1$. On $\partial \Omega \times \partial \Omega$, we have

$$
\begin{gathered}
\left|\partial_{\tau_{z}}^{k} K(\zeta, z)\right| \leq C_{k+1+\alpha}|z-\zeta|^{\alpha-1}, \\
\left|K\left(z_{2}, \zeta\right)-K\left(z_{1}, \zeta\right)\right| \leq C_{1+\alpha} \frac{\left|z_{2}-z_{1}\right|}{\left|\zeta-z_{1}\right|^{2-\alpha}}, \\
\left|\partial_{\tau_{z_{2}}}^{k} K\left(\zeta, z_{2}\right)-\partial_{\tau_{z_{1}}}^{k} K\left(\zeta, z_{1}\right)\right| \leq C_{k+1+\alpha} \frac{\left|z_{2}-z_{1}\right|^{\alpha}}{\left|\zeta-z_{1}\right|},
\end{gathered}
$$

where the last two inequalities require $\left|\zeta-z_{1}\right|>2\left|z_{2}-z_{1}\right|$.

Proof. We first verify (4.2). We have

$$
\partial_{t} \arg (\gamma(s)-\gamma(t))=\frac{N(s, t)}{|\gamma(s)-\gamma(t)|^{2}}, \quad N(s, t)=\nu(t) \cdot \int_{s}^{t}\left(\gamma^{\prime}(r)-\gamma^{\prime}(t)\right) d r .
$$

First, we obtain $\left|N\left(s_{1}, t\right)\right| \leq C\left|t-s_{1}\right|^{1+\alpha}$ and

$$
|| \gamma\left(s_{2}\right)-\left.\gamma(t)\right|^{2}-\left|\gamma\left(s_{1}\right)-\gamma(t)\right|^{2}|\leq C| s_{2}-s_{1} \mid\left(\left|t-s_{1}\right|+\left|t-s_{2}\right|\right) .
$$

Note that

$$
N\left(s_{2}, t\right)-N\left(s_{1}, t\right)=-\nu(t) \cdot \int_{s_{1}}^{s_{2}}\left(\gamma^{\prime}(r)-\gamma^{\prime}(t)\right) d r .
$$

Using $\left|\gamma^{\prime}(r)-\gamma^{\prime}(t)\right| \leq\left|\gamma^{\prime}(r)-\gamma^{\prime}\left(s_{1}\right)\right|+\left|\gamma^{\prime}(t)-\gamma^{\prime}\left(s_{1}\right)\right|$, we obtain

$$
\left|N\left(s_{2}, t\right)-N\left(s_{1}, t\right)\right| \leq C\left(\left|s_{2}-s_{1}\right|^{1+\alpha}+\left|s_{2}-s_{1}\right|\left|t-s_{1}\right|^{\alpha}\right) .
$$

Combining the above, we get for $\left|t-s_{1}\right| \geq 2\left|s_{2}-s_{1}\right|$,

$$
\left|K\left(s_{2}, t\right)-K\left(s_{1}, t\right)\right| \leq C \frac{\left|s_{2}-s_{1}\right|}{\left|t-s_{1}\right|^{2-\alpha}} .
$$

To verify (4.3), we may assume that $x^{\prime}(t) \neq 0$ for $t$ near $s$. For later use, we remark that the rest of the computation does not need $d t$ to be the arc-length element. The condition $x^{\prime}(t) \neq 0$ is only to ensure that $C^{-1}|t-s| \leq|x(t)-x(s)| \leq$ $C|t-s|$. Following [8], let

$$
(x(t)-x(s)) q(s, t)=y(t)-y(s) .
$$

By (2.7), we have $\partial_{\tau} u(\gamma(t))=\left|\partial_{t} \gamma\right|^{-1} \partial_{t}(u(\gamma(t)))$. By $\arg (x+i y)=\arctan (y / x)$ $\bmod \pi$, we get

$$
\partial_{\tau}^{k} K(\gamma(s), \gamma(t))=\sum_{j \leq k} Q_{j}^{\lambda}(t) \partial_{t}^{j} q(s, t),
$$


where $Q_{j}$ are $C^{\infty}$ functions in $\left|\partial_{t} \gamma\right|^{-1}, \partial_{t} \gamma, \ldots, \partial_{t}^{k+1} \gamma$, and $q(s, t)$. Hence (4.3) follows from

$$
\left|\partial_{t_{2}}^{k} q\left(s, t_{2}\right)-\partial_{t_{1}}^{k} q\left(s, t_{1}\right)\right| \leq C_{k+1+\alpha} \frac{\left|t_{2}-t_{1}\right|^{\alpha}}{\left|s-t_{1}\right|} .
$$

Differentiate the equation and solve for $\partial_{t}^{j} q$. Then $(x(t)-x(s))^{k+2} \partial_{t}^{k+1} q$ equals the determinant

$$
\left|\begin{array}{cccccc}
x(t)-x(s) & 0 & 0 & \cdots & 0 & y(t)-y(s) \\
x^{\prime}(t) & x(t)-x(s) & 0 & \cdots & 0 & y^{\prime}(t) \\
x^{\prime \prime}(t) & \left(\begin{array}{c}
2 \\
1
\end{array}\right) x^{\prime}(t) & x(t)-x(s) & \cdots & 0 & y^{\prime \prime}(t) \\
\cdot & \cdot & \cdot & \cdot & \cdot & \cdot \\
\cdot & \cdot & \cdot & \cdot & \cdot & \cdot \\
\cdot & \cdot & \cdot & \cdot & \cdot & \cdot \\
x^{(k)}(t) & \left(\begin{array}{c}
k \\
1
\end{array}\right) x^{(k-1)}(t) & \left(\begin{array}{c}
k \\
2
\end{array}\right) x^{(k-2)}(t) & \cdots & x(t)-x(s) & y^{(k)}(t) \\
x^{(k+1)}(t) & \left(\begin{array}{c}
k+1 \\
1
\end{array}\right) x^{(k)}(t) & \left(\begin{array}{c}
k+1 \\
2
\end{array}\right) x^{(k-1)}(t) & \cdots & \left(\begin{array}{c}
k+1 \\
k
\end{array}\right) x^{\prime}(t) & y^{(k+1)}(t)
\end{array}\right| .
$$

Multiply the $i$-th row by $\frac{1}{i !}(s-t)^{i}$ and add it to the first row. The entries in the first row become

$$
\frac{1}{j !}(s-t)^{j}\left(\mathcal{P}_{k+1-j} x(s, t)-x(s)\right), \quad 0 \leq j \leq k, \quad \mathcal{P}_{k+1} y(s, t)-y(s),
$$

where $\mathcal{P}_{k} f(s, t)$ denotes the Taylor polynomial of degree $k$ of $f$ about $s=t$. Then the remainder $\mathcal{R}_{k} f(s, t)=f(s)-\mathcal{P}_{k} f(s, t)$ can be written as

$$
\mathcal{R}_{k} f(s, t)=\frac{(s-t)^{k}}{k !} \hat{R}_{k} f(s, t), \quad \hat{R}_{k} f(s, t)=\int_{0}^{1}\left\{f^{(k)}(t+r(s-t))-f^{(k)}(t)\right\} d r .
$$

Therefore,

$$
(x(s)-x(t))^{k+2} \partial_{t}^{k+1} q(s, t)=(s-t)^{k+1}\left\{P_{0} \hat{R}_{k+1} y(s, t)+\sum_{i=1}^{k+1} P_{i} \hat{R}_{i} x(s, t)\right\},
$$

where $P_{i}(s, t)$ are polynomials in $\partial_{t} \gamma, \ldots, \partial_{t}^{k+1} \gamma, x(s)-x(t)$. Then (4.1) follows from $\left|\hat{R}_{k+1} \gamma(s, t)\right| \leq C|\gamma|_{k+1+\alpha}|s-t|^{\alpha}$. Assume that $\left|s-t_{2}\right| \geq 2\left|t_{2}-t_{1}\right|$. We have $\left|\hat{R}_{i} \gamma\left(s, t_{2}\right)\right| \leq C\left|s-t_{1}\right|^{\alpha}$ and

$$
\left|\left(P_{i}, \hat{R}_{k+1} \gamma\right)\left(s, t_{2}\right)-\left(P_{i}, \hat{R}_{k+1} \gamma\right)\left(s, t_{1}\right)\right| \leq C|\gamma|_{k+1+\alpha}\left|t_{2}-t_{1}\right|^{\alpha} .
$$

Using $\left|t_{2}-t_{1}\right| \leq\left|s-t_{1}\right|^{1-\alpha}\left|t_{2}-t_{1}\right|^{\alpha}$, we get

$$
\begin{gathered}
\left|\left(s-t_{2}\right)^{k+1}-\left(s-t_{1}\right)^{k+1}\right| \leq C\left|s-t_{1}\right|^{k+1-\alpha}\left|t_{2}-t_{1}\right|^{\alpha}, \\
\left|\left(x(s)-x\left(t_{2}\right)\right)^{-k-2}-\left(x(s)-x\left(t_{1}\right)\right)^{-k-2}\right| \leq C\left|s-t_{1}\right|^{-k-2-\alpha}\left|t_{2}-t_{1}\right|^{\alpha} .
\end{gathered}
$$

By the above inequalities, we get (4.4) and hence (4.3).

We need a function $\Theta$ which plays an important role in Kellogg's first-order derivative estimate. Define a single-valued continuous function $\pi \Theta(t, t)$ on $[0, l]$, which measures the angle from the $x$-axis to the tangent line of $\partial \Omega$ at $\gamma(t)$. Set

$$
\Theta(s, t)=\Theta(s, s)+\frac{1}{\pi} \int_{s}^{t} \partial_{r} \arg (\gamma(s)-\gamma(r)) d r, \quad s, t \in[0, l] .
$$

Then $\partial_{t} \Theta(s, t)=K(s, t), \Theta(s, t)=\Theta(t, s)$, and $\Theta(s, l)-\Theta(s, 0)=1$. 
Lemma 4.2. Let $\partial \Omega \in \mathcal{C}^{k+1+\alpha}$ with $k \geq 0$ and $0<\alpha<1$. Let $I_{i}=\left(l_{0}+\cdots+\right.$ $\left.l_{i-1}, l_{0}+\cdots+l_{i}\right)$.

(i) Let $\varphi \in L^{1}(\partial \Omega)$. In the sense of distributions,

$$
\begin{aligned}
& \partial_{s} \int_{0}^{l} \varphi(\gamma(t)) \Theta(s, t) d t=\int_{0}^{l} \varphi(\gamma(t)) K(\gamma(t), \gamma(s)) d t, \quad s \in I_{i}, \\
& \partial_{\tau}^{k} \int_{\partial \Omega} \varphi(\zeta) K(\zeta, z) d \sigma(\zeta)=\int_{\partial \Omega} \varphi(\zeta) \partial_{\tau_{z}}^{k} K(\zeta, z) d \sigma(\zeta), \quad z \in \partial \Omega .
\end{aligned}
$$

(ii) If $\varphi$ and $\partial_{\tau} \varphi$ are in $L^{1}(\partial \Omega)$, then on $\partial \Omega$ and in the sense of distributions,

$$
\partial_{\tau} \int_{\partial \Omega} \varphi(\zeta) K(z, \zeta) d \sigma(\zeta)=-\int_{\partial \Omega} \partial_{\tau} \varphi(\zeta) K(\zeta, z) d \sigma(\zeta)
$$

Proof. (i) Note that $\Theta(s, t)$ is a continuous branch of $\frac{1}{\pi} \arctan \frac{y(s)-y(t)}{x(s)-x(t)}$ on $[0, l] \times$ $[0, l]$. Then

$$
\partial_{s} \int_{I_{j}} \varphi(t) \Theta(s, t) d t=\int_{I_{j}} \varphi(t) \partial_{s} \arg (\gamma(s)-\gamma(t)) d t
$$

holds on $I_{i}$ when $j \neq i$. It suffices to verify that on $I_{i}$,

$$
\partial_{s} \int_{I_{i}} \varphi(t) \Theta(s, t) d t=\int_{I_{i}} \varphi(t) \partial_{s} \arg (\gamma(s)-\gamma(t)) d t .
$$

Thus we may assume that $\partial \Omega=\gamma_{i}$. We have

$$
\int_{0}^{l} \int_{0}^{l} \frac{|\varphi(t)|}{|t-s|^{1-\alpha}} d t d s \leq C|\varphi|_{L^{1}}
$$

Hence,

$$
\int_{0}^{l} \int_{0}^{l}\left|\varphi(t) \partial_{s} \Theta(s, t)\right| d t d s \leq C \int_{0}^{l} \int_{0}^{l} \frac{|\varphi(t)|}{|t-s|^{1-\alpha}} d t d s \leq C^{\prime}|\varphi|_{L^{1}} .
$$

Therefore, $\int_{0}^{l}\left|\varphi(s) \partial_{s} \Theta(s, t)\right| d s$ is in $L^{1}(\partial \Omega)$. For a test function $\phi$ on $(0, l)$,

$$
\begin{aligned}
\int_{0}^{l} \phi^{\prime}(s) \int_{0}^{l} \varphi(t) \Theta(s, t) d t d s & =\int_{0}^{l} \varphi(t) \int_{0}^{l} \phi^{\prime}(s) \Theta(s, t) d s d t \\
& =-\int_{0}^{l} \phi(s) \int_{0}^{l} \varphi(t) \partial_{s} \Theta(s, t) d t d s
\end{aligned}
$$

which gives us (4.6).

To verify (4.7), we let $k \geq 1$ and use

$$
\partial_{\tau}^{k-1} \int \varphi(\zeta) K(\zeta, z) d \sigma(\zeta)=\int \varphi(\zeta) \partial_{\tau_{z}}^{k-1} K(\zeta, z) d \sigma(\zeta)
$$

Let $\phi$ be a $\mathcal{C}^{1}$ function on $\partial \Omega$. Let $\chi_{\epsilon}(\zeta, z)-1$ be a $\mathcal{C}^{1}$ function on $\partial \Omega \times \partial \Omega$ which has support in $|\zeta-z|<\epsilon$ such that $\left|\chi_{\epsilon}\right| \leq 1$ and $\left|\partial_{\tau_{z}} \chi_{\epsilon}(\zeta, z)\right|<C \epsilon^{-1}$. Now

$$
\begin{aligned}
I & =\int \partial_{\tau_{z}} \phi(z) \int \varphi(\zeta) \partial_{\tau_{z}}^{k-1} K(\zeta, z) d \sigma(\zeta) d \sigma(z) \\
& =\lim _{\epsilon \rightarrow 0} \int \varphi(\zeta) \int \chi_{\epsilon}(\zeta, z) \partial_{\tau_{z}}[\phi(z)-\phi(\zeta)] \partial_{\tau_{z}}^{k-1} K(\zeta, z) d \sigma(z) d \sigma(\zeta) .
\end{aligned}
$$


Write the last double integral as $-I_{\epsilon}^{\prime}-I_{\epsilon}^{\prime \prime}$ with

$$
\begin{gathered}
I_{\epsilon}^{\prime}=\int \varphi(\zeta) \int\left(\partial_{\tau_{z}} \chi_{\epsilon}(\zeta, z)\right)[\phi(z)-\phi(\zeta)] \partial_{\tau_{z}}^{k-1} K(\zeta, z) d \sigma(z) d \sigma(\zeta), \\
I_{e}^{\prime \prime}=\int \varphi(\zeta) \int \chi_{\epsilon}(\zeta, z)[\phi(z)-\phi(\zeta)] \partial_{\tau_{z}}^{k} K(\zeta, z) d \sigma(z) d \sigma(\zeta) .
\end{gathered}
$$

Then $I_{\epsilon}^{\prime}$ tends to 0 uniformly in $\zeta$ as $\epsilon \rightarrow 0$ because as $\epsilon$ tends to 0 ,

$$
\begin{aligned}
& \left|\int\left(\partial_{\tau_{z}} \chi_{\epsilon}(\zeta, z)\right)[\phi(z)-\phi(\zeta)] \partial_{\tau_{z}}^{k-1} K(\zeta, z) d \sigma(z)\right| \\
& \quad \leq C \epsilon^{-1} \sup _{|z-\zeta|<\epsilon}|\phi(z)-\phi(\zeta)| \int_{z \in \partial \Omega,|z-\zeta|<\epsilon}\left|\partial_{\tau_{z}}^{k-1} K(\zeta, z)\right| d \sigma(z) \rightarrow 0 .
\end{aligned}
$$

Since $\left|\chi_{\epsilon}\right| \leq 1$ and $\left|(\phi(z)-\phi(\zeta)) \partial_{\tau_{z}}^{k} K(\zeta, z)\right| \leq C$ by (4.1), then $\lim _{\epsilon \rightarrow 0} I_{\epsilon}^{\prime \prime}$ equals

$$
I^{\prime \prime}=\int \varphi(\zeta) \int[\phi(z)-\phi(\zeta)] \partial_{\tau_{z}}^{k} K(\zeta, z) d \sigma(z) d \sigma(\zeta)
$$

Since $k \geq 1$, then

$$
\begin{aligned}
\int \partial_{\tau_{z}}^{k} K(\zeta, z) d \sigma(z) & =\lim _{\epsilon \rightarrow 0} \int_{|z-\zeta|>\epsilon} \partial_{\tau_{z}}^{k} K(\zeta, z) d \sigma(z) \\
& =\lim _{\epsilon \rightarrow 0}\left\{\partial_{\tau_{z}}^{k-1} K\left(\zeta, \zeta_{\epsilon}^{\prime \prime}\right)-\partial_{\tau_{z}}^{k-1} K\left(\zeta, \zeta_{\epsilon}^{\prime}\right)\right\}=0 .
\end{aligned}
$$

Here we have used the continuity of $\partial_{\tau_{z}}^{k-1} K(\zeta, z)$ and the identities $\left\{\zeta_{\epsilon}^{\prime}, \zeta_{\epsilon}^{\prime \prime}\right\}=$ $\partial \Omega \cap\{z:|z-\zeta|=\epsilon\}$ for small $\epsilon$ and $\lim _{\epsilon \rightarrow 0} \zeta_{\epsilon}^{\prime}=z=\lim _{\epsilon \rightarrow 0} \zeta_{\epsilon}^{\prime \prime}$. Now (4.7) follows from

$$
I=-\int \varphi(\zeta) \int \phi(z) \partial_{\tau_{z}}^{k} K(\zeta, z) d \sigma(z) d \sigma(\zeta)
$$

(ii) When $\partial \Omega$ is parameterized by $\gamma(t)$, at $z=\gamma(t)$, we have $\partial_{\tau} f(z) d \sigma(z)=$ $d f(z)=\partial_{t}(f(\gamma(t))) d t$. Then (4.8) is obtained by integration by parts and (4.6).

We have seen from (4.8) that differentiating integral operator $\mathcal{K}$ inevitably leads to the kernel $\mathcal{K}^{*}$. To recover a loss of regularity in Kellogg's arguments, we will need to combine (4.1) and (4.3) with estimates on $\mathcal{K}, \mathcal{K}^{*}$ from the Cauchy transform.

Lemma 4.3. Let $\partial \Omega \in \mathcal{C}^{k+1+\alpha}$ with $0<\alpha<1$. Then for a real function $\psi \in$ $\mathcal{C}^{\alpha}(\partial \Omega)$

$$
\mathcal{K} \psi=2 \operatorname{Re}\left\{\mathcal{C}^{+} \psi\right\}-\psi, \quad \mathcal{K}^{*} \psi=\psi-2 \operatorname{Re}\left\{\tau \mathcal{C}^{+}(\bar{\tau} \psi)\right\}
$$

In particular, $\mathcal{K}^{*}\left(\mathcal{C}^{k+\alpha}(\partial \Omega)\right) \subset \mathcal{C}^{k+\alpha}(\partial \Omega)$, and for $l \leq k+1, \mathcal{K}\left(\mathcal{C}^{l+\alpha}(\partial \Omega)\right) \subset$ $\mathcal{C}^{l+\alpha}(\partial \Omega)$.

Proof. The first formula follows from (3.11) immediately. Parameterize $\partial \Omega$ by $\gamma$ in arc-length. By a simple computation we obtain

$$
\begin{aligned}
\partial_{s} \arg (\gamma(s)-\gamma(t))= & -\operatorname{Re}\left(\gamma^{\prime}(s) \overline{\gamma^{\prime}(t)}\right) \partial_{t} \arg (\gamma(s)-\gamma(t)) \\
& -\operatorname{Im}\left(\gamma^{\prime}(s) \overline{\gamma^{\prime}(t)}\right) \partial_{t} \log |\gamma(s)-\gamma(t)| .
\end{aligned}
$$

To verify the second one, we use (4.9) to decompose

$$
\mathcal{K}^{*} \psi(z)=\frac{1}{\pi} \int_{\partial \Omega} \psi(\zeta) \partial_{\tau_{z}} \arg (z-\zeta) d \sigma(\zeta)=J_{1}(z)+J_{2}(z)
$$


with

$$
\begin{gathered}
J_{1}(z)=-\psi(z)+\frac{1}{\pi} \operatorname{Re}\left\{\tau_{z} \int_{\partial \Omega}\left(\psi(z) \overline{\tau_{z}}-\psi(\zeta) \overline{\tau_{\zeta}}\right) \partial_{\tau_{\zeta}} \arg (z-\zeta) d \sigma(\zeta)\right\}, \\
J_{2}(z)=\frac{1}{\pi} \operatorname{Im}\left\{\tau_{z} \int_{\partial \Omega}\left(\psi(z) \overline{\tau_{z}}-\psi(\zeta) \overline{\tau_{\zeta}}\right) \partial_{\tau_{\zeta}} \log |z-\zeta| d \sigma(\zeta)\right\}
\end{gathered}
$$

By a simple computation,

$$
\mathcal{K}^{*} \psi(z)=-\psi(z)+\frac{1}{\pi} \operatorname{Im}\left\{\tau_{z} \int_{\partial \Omega}\left(\psi(z) \overline{\tau_{z}}-\psi(\zeta) \overline{\tau_{\zeta}}\right) \frac{d \zeta}{\zeta-z}\right\}
$$

Therefore, $\mathcal{K}^{*} \psi=-\psi-2 \operatorname{Re}\left\{\tau \mathcal{C}^{-}(\bar{\tau} \psi)\right\}$. The assertions follow from Lemma 3.2 .

\section{KeRnels With PARAMETER}

We have derived estimates for $\mathcal{K}, \mathcal{K}^{*}$ and $\mathcal{C} f$. In this section we modify the arguments for the parameter case. The requirement that $k \geq j$ in the Hölder spaces $\mathcal{C}^{k+\alpha, j}(\partial \Omega)$ will be evident in identity (5.17) below and in the proof of Lemma 5.4 for the Cauchy transform with parameter.

Lemma 5.1. Let $\Omega$ be a bounded domain in $\mathbf{C}$ with $\partial \Omega \in \mathcal{C}^{1}$. Let $\Gamma^{\lambda}$ embed $\bar{\Omega}$ onto $\overline{\Omega^{\lambda}}$ with $\Gamma \in \mathcal{C}_{*}^{1, j}(\bar{\Omega})$. Let $z^{\lambda}=\Gamma^{\lambda}(z)$ and $k>0$. For $z, \zeta \in \bar{\Omega}$ with $\zeta \neq z$,

$$
\begin{gathered}
\left|\frac{1}{\left(\zeta^{\mu}-z^{\mu}\right)^{k}}-\frac{1}{\left(\zeta^{\lambda}-z^{\lambda}\right)^{k}}\right| \leq C_{1,0} \frac{\left|\Gamma^{\mu}-\Gamma^{\lambda}\right|_{1}}{|\zeta-z|^{k}}, \\
\left|\frac{1}{\left(x^{\mu}(\zeta)-x^{\mu}(z)\right)^{k}}-\frac{1}{\left(x^{\lambda}(\zeta)-x^{\lambda}(z)\right)^{k}}\right| \leq C_{*} C_{1,0} \frac{\left|x^{\mu}-x^{\lambda}\right|_{1}}{|\zeta-z|^{k}}, \\
\left|\partial_{\lambda}^{j} \log \right| \zeta^{\lambda}-z^{\lambda}|| \leq C_{1, j}^{*}, \quad j \geq 1, \\
\left|\partial_{\mu}^{j} \log \right| \zeta^{\mu}-z^{\mu}\left|-\partial_{\lambda}^{j} \log \right| \zeta^{\lambda}-z^{\lambda}|| \leq C_{1, j}^{*}\left|\Gamma^{\mu}-\Gamma^{\lambda}\right|_{1, j}, \quad j \geq 0,
\end{gathered}
$$

where (5.2) is for $\zeta, z \in \partial \Omega$ and under the assumptions that $\left|\partial_{\tau_{z}} x^{\lambda}\right| \geq 1 / C_{*}$ and $\left|x^{\mu}-x^{\lambda}\right|_{1},|\zeta-z|$ are sufficiently small. Assume further that $\Gamma \in \mathcal{B}_{*}^{1+\alpha, j}(\partial \Omega)$. Then for $\zeta \in \partial \Omega$,

$$
\left|\partial_{\lambda}^{j} \partial_{\tau_{\zeta}^{\lambda}} \arg \left(\zeta^{\lambda}-z^{\lambda}\right)\right| \leq C_{1+\alpha, j}^{*} \frac{|\zeta-z|^{1+\alpha}+\operatorname{dist}(z, \partial \Omega)}{|\zeta-z|^{2}} .
$$

Proof. Since $\Gamma^{\lambda}$ are embeddings with $\Gamma \in \mathcal{C}^{1,0}(\bar{\Omega})$, we have

$$
|\zeta-z| / C \leq\left|\zeta^{\lambda}-z^{\lambda}\right| \leq C|\zeta-z| \text {. }
$$

Take a path $\rho$ in $\bar{\Omega}$ such that $\rho(0)=z, \rho(1)=\zeta$ and $\left|\rho^{\prime}\right| \leq C|\zeta-z|$. When $j=0$, (5.4) follows from $|\log (1+x)| \leq 2|x|$ for $|x|<1 / 2$ and

$$
\begin{aligned}
\left|\partial_{\mu}^{j}\left(\zeta^{\mu}-z^{\mu}\right)-\partial_{\lambda}^{j}\left(\zeta^{\lambda}-z^{\lambda}\right)\right| & =\left|\int_{0}^{1} \nabla\left(\partial_{\mu}^{j} \Gamma^{\mu}-\partial_{\lambda}^{j} \Gamma^{\lambda}\right)(\rho(t)) \cdot \rho^{\prime}(t) d t\right| \\
& \leq C\left|\Gamma^{\mu}-\Gamma^{\lambda}\right|_{1, j}|\zeta-z|,
\end{aligned}
$$

and (5.1) follows from (5.6)-(5.7) too. By analogy, one can verify (5.2). For $j>0$, $\partial_{\lambda}^{j} \log \left|\zeta^{\lambda}-z^{\lambda}\right|$ is a linear combination of

$$
Q^{\lambda}(\zeta, z)=\frac{\partial_{\lambda}^{i_{1}}\left(\zeta^{\lambda}-z^{\lambda}\right) \cdots \partial_{\lambda}^{i_{a}}\left(\zeta^{\lambda}-z^{\lambda}\right)}{\left(\zeta^{\lambda}-z^{\lambda}\right)^{a}}
$$


and their conjugates, where $a>1$ and $i_{l} \leq j$. Using $\left|\partial_{\lambda}^{j}\left(\zeta^{\lambda}-z^{\lambda}\right)\right| \leq C_{1, j}|\zeta-z|$, we obtain (5.4) by (5.7), and (5.3). Note that $Q^{\lambda}$ may not extend continuously to $z=\zeta$.

To verify (5.5), we choose local $\mathcal{C}^{k+1+\alpha}$ coordinates such that $\Omega$ contains $[-1,1] \times$ $(0,1]$ and $\partial \Omega$ contains $[-1,1] \times\{0\}$. Assume that $\zeta=\xi+i 0, z \in \Omega$ and $|\zeta|+|z|<1 / 2$. Then $\gamma_{1}^{\lambda}(x, y)=\partial_{x} \gamma^{\lambda}(x, y)$ is tangent to $\partial \Omega^{\lambda}$ and

$$
\partial_{\tau_{\zeta}^{\lambda}} \arg \left(\zeta^{\lambda}-z^{\lambda}\right)=\left|\gamma_{1}^{\lambda}(\xi, 0)\right|^{-1} \operatorname{Im}\left\{\frac{\gamma_{1}^{\lambda}(\xi, 0) \overline{\left(\gamma^{\lambda}(\xi, 0)-\gamma^{\lambda}(x, y)\right)}}{\left|\gamma^{\lambda}(\xi, 0)-\gamma^{\lambda}(x, y)\right|^{2}}\right\} .
$$

Set $\gamma_{2}^{\lambda}(x, y)=\partial_{y} \gamma^{\lambda}(x, y)$. We have

$$
\begin{aligned}
& \gamma^{\lambda}(x, y)-\gamma^{\lambda}(\xi, 0)=\int_{0}^{1}\left\{(x-\xi) \gamma_{1}^{\lambda}(\xi+r(x-\xi), 0)+y \gamma_{2}^{\lambda}(x, r y)\right\} d r \\
& \operatorname{Im}\left\{\gamma_{1}^{\lambda}(\xi, 0) \overline{\left(\gamma^{\lambda}(\xi, 0)-\gamma^{\lambda}(x, y)\right)}\right\} \\
& =\operatorname{Im}\left\{\overline{\gamma_{1}^{\lambda}(\xi, 0)}\left[(x-\xi) \hat{R}_{1} \gamma_{1}^{\lambda}(x, y, \xi)+y R_{1}^{*} \gamma_{2}^{\lambda}(x, y, \xi)\right]\right\}, \\
& \hat{R}_{1} \gamma_{1}^{\lambda}(x, y, \xi)=\int_{0}^{1}\left[\gamma_{1}^{\lambda}(\xi+r(x-\xi), 0)-\gamma_{1}^{\lambda}(\xi, 0)\right] d r \\
& R_{1}^{*} \gamma_{2}^{\lambda}(x, y, \xi)=\int_{0}^{1} \gamma_{2}^{\lambda}(x, r y) d r
\end{aligned}
$$

Therefore, $\partial_{\lambda}^{j} \partial_{\tau_{\zeta}^{\lambda}} \arg \left(\zeta^{\lambda}-z^{\lambda}\right)$ is a linear combination of

$$
\begin{aligned}
& \partial_{\lambda}^{i_{0}}\left|\gamma_{1}^{\lambda}(\xi, 0)\right|^{-1} \operatorname{Im}\left\{\overline { \partial _ { \lambda } ^ { i _ { 1 } } \gamma _ { 1 } ^ { \lambda } ( \xi , 0 ) } \left[(x-\xi) \hat{R}_{1} \partial_{\lambda}^{i_{2}} \gamma_{1}^{\lambda}(x, y, \xi)\right.\right. \\
& \left.\left.+y R_{1}^{*} \partial_{\lambda}^{i_{2}} \gamma_{2}^{\lambda}(x, y, \xi)\right]\right\} q_{i_{3}}^{\lambda}(\xi, x, y) .
\end{aligned}
$$

Here $i_{0}+i_{1}+i_{2}+i_{3}=j$ and $q_{i_{3}}^{\lambda}(\xi, x, y)=\partial_{\lambda}^{i_{3}}\left|\gamma^{\lambda}(\xi, 0)-\gamma^{\lambda}(x, y)\right|^{-2}$. We can verify that

$$
\left.\left|\partial_{\lambda}^{i_{0}}\right| \gamma_{1}^{\lambda}(\xi, 0)\right|^{-1}\left|\leq C_{1, j}, \quad\right| \partial_{\lambda}^{i_{1}} \gamma_{1}^{\lambda}(\xi, 0) \mid \leq C_{1, j}
$$

By the arguments for (5.3)-(5.4), we obtain

$$
\left|q_{i_{3}}^{\lambda}(\xi, x, y)\right| \leq C|z-\zeta|^{-2} .
$$

By (5.8)-(5.9), we get $\left|\hat{R}_{1} \partial_{\lambda}^{i_{2}} \gamma_{1}^{\lambda}(x, y, \xi)\right| \leq C_{1+\alpha, j}|x-\xi|^{\alpha}$ and $\left|R_{1}^{*} \partial_{\lambda}^{i_{2}} \gamma_{2}^{\lambda}(x, y, \xi)\right| \leq$ $C_{1, j}$. In (5.10), we have $y=\operatorname{dist}(z, \partial \Omega)$ and $|x-\xi| \leq|z-\zeta|$. Combining the above estimates, we get (5.5).

Given a family of continuous functions $f^{\lambda}$ on $\partial \Omega^{\lambda}$, let $\mathcal{C}^{\lambda} f$ be the Cauchy transform defined off $\partial \Omega^{\lambda}$ by (3.10). Let $\mathcal{C}_{+}^{\lambda} f$ be restrictions on $\Omega^{\lambda}$. Denote by $W^{\lambda} f$ and $U^{\lambda} f$ the single and double layer potentials with moment $f^{\lambda}$ on $\partial \Omega^{\lambda}$. Denote by $W_{+}^{\lambda} f$ and $U_{+}^{\lambda} f$ their restrictions on $\Omega^{\lambda}$ and extensions to $\overline{\Omega^{\lambda}}$ if continuous extensions exist. 
It will be convenient to use the notation

$$
\begin{gathered}
\left|u^{\lambda}\right|_{i+\alpha, j}=\max _{l \leq j}\left|\partial_{\lambda}^{l}\left(u^{\lambda} \circ \Gamma^{\lambda}\right)\right|_{i+\alpha}, \quad\left\|u^{\lambda}\right\|_{k+\alpha, j}=\max _{i \leq j}\left|u^{\lambda}\right|_{k+\alpha-i, i}, \\
\left|u^{\mu}-u^{\lambda}\right|_{i+\alpha, j}=\max _{l \leq j}\left|\partial_{\mu}^{l}\left(u^{\mu} \circ \Gamma^{\mu}\right)-\partial_{\lambda}^{l}\left(u^{\lambda} \circ \Gamma^{\lambda}\right)\right|_{i+\alpha}, \\
\left\|u^{\mu}-u^{\lambda}\right\|_{k+\alpha, j}=\max _{i \leq j}\left|u^{\mu}-u^{\lambda}\right|_{k-i+\alpha, i}, \quad j \leq k .
\end{gathered}
$$

Define analogous norms by replacing $\Gamma^{\lambda}$ with $\gamma^{\lambda}$.

Proposition 5.2. Let $\Gamma^{\lambda}$ embed $\bar{\Omega}$ onto $\overline{\Omega^{\lambda}}$ with $\Gamma \in \mathcal{B}^{k+1+\alpha, j}(\bar{\Omega})$. Let $j \leq k$ and $j \leq l \leq k+1$. With the norms defined by (2.1)-(2.3) and (5.11)-(5.13), we have

$$
\begin{gathered}
\left\|\mathcal{C}_{+} f\right\|_{0,0} \leq C_{1,0}|f|_{\alpha, 0}, \quad\left\|\mathcal{C}_{+} f\right\|_{l+\alpha, j} \leq C_{k+1+\alpha, j}\|f\|_{l+\alpha, j}, \\
\left\|\mathcal{C}_{+}^{\mu} f-\mathcal{C}_{+}^{\lambda} f\right\|_{0,0} \leq C_{1,0}\left(\left|\Gamma^{\mu}-\Gamma^{\lambda}\right|_{1}\left|f^{\lambda}\right|_{\alpha}+\left\|f^{\mu}-f^{\lambda}\right\|_{\alpha, 0}\right), \\
\left\|\mathcal{C}_{+}^{\mu} f-\mathcal{C}_{+}^{\lambda} f\right\|_{l+\alpha, j} \leq C_{k+1+\alpha, j}\left(\left\|\Gamma^{\mu}-\Gamma^{\lambda}\right\|_{k+1+\alpha, j}\left|f^{\lambda}\right|_{l+\alpha}\right. \\
\left.+\left\|f^{\mu}-f^{\lambda}\right\|_{l+\alpha, j}\right) .
\end{gathered}
$$

If $\partial \Omega$ is real analytic, and $\Gamma^{\lambda}(z)$ and $f^{\lambda} \circ \Gamma^{\lambda}(z)$ are real analytic on $\bar{\Omega} \times[0,1]$, then $\mathcal{C}_{+}^{\lambda} f \circ \Gamma^{\lambda}(z)$ and $W_{+}^{\lambda} f \circ \Gamma^{\lambda}(z)$ are real analytic on $\bar{\Omega} \times[0,1]$ too.

Proof. Let $z \in \Omega$. Take $z_{*} \in \partial \Omega$ such that $\left|z_{*}-z\right|=\operatorname{dist}(z, \partial \Omega)$. We have

$$
\mathcal{C}^{\lambda} f\left(z^{\lambda}\right)=f^{\lambda}\left(z_{*}^{\lambda}\right)+\frac{1}{2 \pi i} \int_{\partial \Omega} \frac{f^{\lambda}\left(\zeta^{\lambda}\right)-f^{\lambda}\left(z_{*}^{\lambda}\right)}{\zeta^{\lambda}-z^{\lambda}} d \zeta^{\lambda} .
$$

Denote the last integral by $A^{\lambda}(z)$. By (5.1) it is easy to see that

$$
\left|A^{\mu}-A^{\lambda}\right|_{0} \leq C_{1,0}\left(\left\|f^{\mu}-f^{\lambda}\right\|_{\alpha, 0}+\left|f^{\lambda}\right|_{\alpha}\left|\Gamma^{\mu}-\Gamma^{\lambda}\right|_{1}\right) \int_{\partial \Omega} \frac{\left|\zeta-z_{*}\right|^{\alpha}}{|\zeta-z|}|d \zeta| .
$$

The last integral is bounded by a constant; indeed, when $\delta=\operatorname{dist}(z, \partial \Omega)$ is sufficiently small, for $z_{*}=\gamma(s)$ and $\zeta=\gamma(t)$ we have $\left|\zeta-z_{*}\right| \leq C|s-t|$ and $|z-\zeta| \geq(\delta+|t-s|) / C$. This verifies (5.15). By Lemma $3.2, \mathcal{C}_{+}^{\lambda} f$ is continuous when $\lambda$ is fixed. Then (5.15) also implies that $\mathcal{C}_{+} f$ is in $\mathcal{C}^{0,0}\left(\bar{\Omega}_{\Gamma}\right)$. One can also verify the first inequality in (5.14). Notice that the proof merely needs $\Gamma \in \mathcal{C}^{1,0}(\bar{\Omega})$. Next, we will verify (5.16) and the second inequality in (5.14).

Denote by $\partial_{z^{\lambda}}$ the derivative in $z$ on $\Omega^{\lambda}$. By analogue of Lemma 2.4, it suffices to estimate norms for $\partial_{z^{\lambda}} \mathcal{C}_{+}^{\lambda} f$. We first consider the case where $j=0$. Differentiate $\mathcal{C}^{\lambda} f$ and then apply Stokes to transport the derivatives to $f^{\lambda}\left(\gamma^{\lambda}(\zeta)\right)$. By (3.12) we have

$$
g^{\lambda}\left(z^{\lambda}\right) \stackrel{\text { def }}{=} \partial_{z^{\lambda}}^{l} \mathcal{C}^{\lambda} f\left(z^{\lambda}\right)=\frac{1}{2 \pi i} \int_{\zeta \in \partial \Omega} \frac{\tilde{\partial}_{\tau^{\lambda}}^{l} f^{\lambda}\left(\zeta^{\lambda}\right)}{\zeta^{\lambda}-z^{\lambda}} d \zeta^{\lambda} \stackrel{\text { def }}{=} \int_{\zeta \in \partial \Omega} \frac{h^{\lambda}\left(\zeta^{\lambda}\right) d \zeta^{\lambda}}{\zeta^{\lambda}-z^{\lambda}},
$$

where $\tilde{\partial}_{\tau^{\lambda}}=\overline{\tau^{\lambda}} \partial_{\tau^{\lambda}}$. We have

$$
\partial_{z} g^{\lambda}\left(z^{\lambda}\right)=\partial_{z} \Gamma^{\lambda}(z) I^{\lambda}(z), \quad I^{\lambda}(z)=\int_{\partial \Omega} \frac{h^{\lambda}\left(\zeta^{\lambda}\right)-h^{\lambda}\left(z_{*}^{\lambda}\right)}{\left(\zeta^{\lambda}-z^{\lambda}\right)^{2}} d \zeta^{\lambda} .
$$


Note that by the product rule, $\tilde{\partial}_{\tau^{\lambda}}^{l} f^{\lambda}\left(\zeta^{\lambda}\right)$ involves derivatives of order at most $l$ in $\zeta^{\lambda}$ and $f^{\lambda}\left(\zeta^{\lambda}\right)$. Thus $\left|h^{\lambda}\right|_{\alpha} \leq C_{k+1+\alpha}\left|f^{\lambda}\right|_{l+\alpha}$. We can verify that

$$
\begin{aligned}
\left|I^{\lambda}(z)\right| & \leq C\left|h^{\lambda}\right|_{\alpha} \int_{\partial \Omega}\left|\zeta^{\lambda}-z^{\lambda}\right|^{\alpha-2} d \sigma \leq C^{\prime}\left|h^{\lambda}\right|_{\alpha} \int_{\partial \Omega}|\zeta-z|^{\alpha-2} d \sigma \\
& \leq C_{k+1+\alpha}\left|f^{\lambda}\right|_{l+\alpha} \operatorname{dist}(z, \partial \Omega)^{\alpha-1} .
\end{aligned}
$$

Combining with the first inequality in (5.14), we get its second inequality for $j=0$. Also, by (5.1)

$$
\left|I^{\mu}(z)-I^{\lambda}(z)\right| \leq C\left(\left\|h^{\mu}-h^{\lambda}\right\|_{\alpha, 0}+\left|h^{\lambda}\right|_{\alpha}\left|\Gamma^{\mu}-\Gamma^{\lambda}\right|_{1}\right) \int_{\partial \Omega}|\zeta-z|^{\alpha-2} d \sigma(\zeta) .
$$

Thus, $\left|I^{\mu}(z)-I^{\lambda}(z)\right| \leq C \operatorname{dist}(z, \partial \Omega)^{\alpha-1}$. For $l \leq k+1$ we obtain

$$
\left\|g^{\mu}-g^{\lambda}\right\|_{\alpha, 0} \leq C\left(\left\|h^{\mu}-h^{\lambda}\right\|_{\alpha, 0}+\left|h^{\lambda}\right|_{\alpha}\left|\Gamma^{\mu}-\Gamma^{\lambda}\right|_{k+1+\alpha}\right) .
$$

Note that $\left\|h^{\mu}-h^{\lambda}\right\|_{\alpha, 0} \leq C_{k+1+\alpha}\left(\left\|f^{\mu}-f^{\lambda}\right\|_{k+1+\alpha, j}+\left|f^{\lambda}\right|_{k+1+\alpha}\left|\gamma^{\mu}-\gamma^{\lambda}\right|_{l+\alpha}\right)$ for $l \leq k+1$. This gives us (5.16) for $j=0$.

Assume that $j>0$ and (5.16) is valid when $j$ is replaced by $j-1$. Here we need a crucial cancellation. By (2.6), we have $d \gamma^{\lambda}=\partial_{\tau} \gamma^{\lambda} d \sigma$, i.e., $d \zeta^{\lambda}=\partial_{\tau_{\zeta}} \zeta^{\lambda} d \sigma(\zeta)$. Thus

$$
\begin{aligned}
\partial_{\lambda}\left(\mathcal{C}^{\lambda} f\left(z^{\lambda}\right)\right)= & \frac{1}{2 \pi i} \int_{\zeta \in \partial \Omega} \frac{\partial_{\lambda}\left[\partial_{\tau_{\zeta}} \zeta^{\lambda} f^{\lambda}\left(\zeta^{\lambda}\right)\right]}{\zeta^{\lambda}-z^{\lambda}} d \sigma(\zeta) \\
& -\frac{1}{2 \pi i} \int_{\zeta \in \partial \Omega} \frac{\left(\partial_{\lambda} \zeta^{\lambda}-\partial_{\lambda} z^{\lambda}\right) f^{\lambda}\left(\zeta^{\lambda}\right)}{\left(\zeta^{\lambda}-z^{\lambda}\right)^{2}} d \zeta^{\lambda} .
\end{aligned}
$$

We apply integration by parts to the second term and write the above as

$$
\frac{1}{2 \pi i} \int_{\partial \Omega} \frac{\partial_{\lambda}\left[\partial_{\tau_{\zeta}} \zeta^{\lambda} f^{\lambda}\left(\zeta^{\lambda}\right)\right]}{\zeta^{\lambda}-z^{\lambda}} d \sigma(\zeta)-\frac{1}{2 \pi i} \int_{\partial \Omega} \frac{\partial_{\tau_{\zeta}}\left[\left(\partial_{\lambda} \zeta^{\lambda}-\partial_{\lambda} z^{\lambda}\right) f^{\lambda}\left(\zeta^{\lambda}\right)\right]}{\zeta^{\lambda}-z^{\lambda}} d \sigma(\zeta) .
$$

Cancelling two second-order derivatives, we arrive at

$$
\begin{aligned}
\partial_{\lambda}\left(\mathcal{C}^{\lambda} f\left(z^{\lambda}\right)\right)= & \frac{1}{2 \pi i} \int_{\zeta \in \partial \Omega} \frac{\partial_{\lambda}\left(f^{\lambda}\left(\zeta^{\lambda}\right)\right)-\partial_{\lambda} \zeta^{\lambda}\left(\partial_{\tau_{\zeta}} \zeta^{\lambda}\right)^{-1} \partial_{\tau_{\zeta}}\left(f^{\lambda}\left(\zeta^{\lambda}\right)\right)}{\zeta^{\lambda}-z^{\lambda}} d \zeta^{\lambda} \\
& +\frac{\partial_{\lambda} z^{\lambda}}{2 \pi i} \int_{\zeta \in \partial \Omega} \frac{\left(\partial_{\tau_{\zeta}} \zeta^{\lambda}\right)^{-1} \partial_{\tau_{\zeta}}\left(f^{\lambda}\left(\zeta^{\lambda}\right)\right)}{\zeta^{\lambda}-z^{\lambda}} d \zeta^{\lambda} .
\end{aligned}
$$

Now (5.16) follows from the induction hypothesis where $(j, l)$ is replaced with $(j-$ $1, l-1)$. By a simpler computation, estimates (5.14) for $j>0$ follow from (5.17) too.

The proof for real analyticity in Lemma 3.2 cannot be applied to the parameter case, as generally we cannot normalize two differential operators simultaneously. Instead, we will prove it by estimating Taylor coefficients. We start with

$$
\partial_{z}\left(\mathcal{C}^{\lambda} f\left(z^{\lambda}\right)\right)=\frac{\partial_{z} z^{\lambda}}{2 \pi i} \int_{\zeta \in \partial \Omega} \frac{\overline{\tau_{\zeta}} \partial_{\tau}\left(f^{\lambda}\left(\zeta^{\lambda}\right)\right)}{\zeta^{\lambda}-z^{\lambda}} d \zeta^{\lambda} .
$$

An analogous formula holds for $\partial_{\bar{z}}\left(\mathcal{C}^{\lambda} f\left(z^{\lambda}\right)\right)$. By (5.17)-(5.18), we express

$$
\partial_{z}^{i} \partial_{\bar{z}}^{j} \partial_{\lambda}^{k-i-j} \mathcal{C}_{+}^{\lambda} f^{\lambda}\left(z^{\lambda}\right)=\sum_{l=1}^{N_{i, j ; k}} P_{i, j, k, l}^{\lambda}\left(z^{\lambda}\right) \mathcal{C}_{+}^{\lambda}\left\{Q_{i, j, k, l}^{\lambda}\left(\zeta^{\lambda}\right)\right\}\left(z^{\lambda}\right)
$$


Here $P_{i, j, k, l}^{\lambda}\left(z^{\lambda}\right) Q_{i, j, k, l}^{\lambda}\left(\zeta^{\lambda}\right)$ is the product of elements of the form

$$
\begin{gathered}
\partial_{z}^{a_{1}} \partial_{\bar{z}}^{b_{1}} \partial_{\lambda}^{c_{1}+1} z^{\lambda}, \quad \partial_{z}^{a_{2}+1} \partial_{\bar{z}}^{b_{2}} \partial_{\lambda}^{c_{2}} z^{\lambda}, \quad \partial_{z}^{a_{3}} \partial_{\bar{z}}^{b_{3}+1} \partial_{\lambda}^{c_{3}} z^{\lambda} ; \\
\partial_{\tau}^{a_{4}} \partial_{\lambda}^{b_{4}+1} \zeta^{\lambda}, \quad \partial_{\tau}^{a_{5}} \partial_{\lambda}^{b_{5}}\left(\partial_{\tau} \zeta^{\lambda}\right)^{-1}, \quad \partial_{\tau}^{a_{6}} \overline{\tau_{\zeta}} ; \\
\partial_{\tau}^{a_{7}} \partial_{\lambda}^{b_{7}}\left(f^{\lambda}\left(\zeta^{\lambda}\right)\right) ; \quad L(\zeta, z, \lambda)=\left(\partial_{\lambda} z^{\lambda}, \partial_{z} z^{\lambda}, \partial_{\bar{z}} z^{\lambda}, \partial_{\lambda} \zeta^{\lambda},\left(\partial_{\tau} \zeta^{\lambda}\right)^{-1}, \overline{\tau_{\zeta}}\right) .
\end{gathered}
$$

Let us explain how the above terms are used. Let $L$ denote all first-order derivatives appearing in the right-hand sides of (5.17)-(5.18) and $\partial_{\bar{z}} z^{\lambda}$ in the formulae analogous to (5.18), except those of $f$. To count the total of the orders of derivatives efficiently, we will count the first-order derivatives appearing in $L$ separately, when (5.17) or (5.18) is used each time. Set $b_{6}=c_{4}=\cdots=c_{7}=0$. For the purpose of counting, we duplicate the above terms associated to $\left(a_{n}, b_{n}, c_{n}\right)$ for $n<7$ and denote by $m_{n}$ the number of the copies associated to $\left(a_{n}, b_{n}, c_{n}\right)$ that appear in $P_{i, j, k, l}^{\lambda} Q_{i, j, k, l}^{\lambda}$. Since $\partial_{\tau}^{a_{7}} \partial_{\lambda}^{b_{7}}\left(f^{\lambda}\left(\zeta^{\lambda}\right)\right)$ appears once in $P_{i, j, k, l}^{\lambda} Q_{i, j, k, l}^{\lambda}$, we set $m_{7}=1$. By an abuse of notation, we have not expressed the dependence of $m_{n}$ on $i, j, k, l$, $a_{n^{\prime}}, b_{n^{\prime}}$ and $c_{n^{\prime}}$. Nevertheless, we have

$$
d_{k}=\max _{i, j, l} \sum_{n=1}^{7} m_{n}\left(a_{n}+b_{n}+c_{n}\right) \leq k, \quad \prod_{n=1}^{7}\left(a_{n} ! b_{n} ! c_{n} !\right)^{m_{n}} \leq k ! .
$$

Since $z^{\lambda}$ and $f^{\lambda}\left(\zeta^{\lambda}\right)$ are real analytic, we have

$$
\begin{gathered}
|L| \leq C_{0}, \quad\left|\partial_{z}^{a} \partial_{\bar{z}}^{b} \partial_{\lambda}^{c} z^{\lambda}\right| \leq(a+b+c-1) ! C_{0}^{a+b+c}, \quad a+b+c>0, \\
\left|\partial_{\tau}^{a} \partial_{\lambda}^{b}\left(\partial_{\lambda} \zeta^{\lambda},\left(\partial_{\tau} \zeta^{\lambda}\right)^{-1}, \overline{\tau_{\zeta}}, f^{\lambda}\left(\zeta^{\lambda}\right)\right)\right| \leq(a+b) ! C_{0}^{a+b+1} .
\end{gathered}
$$

Here the last inequality is obtained by using real analytic parameterization in arclength. Thus, the product of the terms in $P_{i, j, k, l}^{\lambda} Q_{i, j, k, l}^{\lambda}$, excluding those in $L$, is bounded in sup norm by

$$
\prod_{n=1}^{7}\left(a_{n} ! b_{n} ! c_{n} !\right)^{m_{n}} C_{0}^{m_{n}\left(a_{n}+b_{n}+c_{n}\right)} \leq C_{0}^{k} k ! .
$$

Next, we count $l_{k}$, the maximum number of first-order derivatives in $L$ which appear in each $P_{i, j, k, l}^{\lambda} Q_{i, j, k, l}^{\lambda}$ as $i, j$ and $l$ vary. From (5.17), taking one derivative in $\partial_{\lambda}$ produces at most two terms in $L$; from (5.18), taking one derivative in $z$ or $\bar{z}$ produces two terms in $L$. Therefore, $l_{k} \leq 2 k+1$. Thus, the product of the terms in $L$ that appear in $P_{i, j, k, l}^{\lambda} Q_{i, j, k, l}^{\lambda}$ is bounded in sup norm by $C_{0}^{l_{k}} \leq C_{0}^{2 k+1}$. Combining with (5.20), we get

$$
\left|P_{i, j, k, l}^{\lambda} Q_{i, j, k, l}^{\lambda}\right|_{0} \leq C_{0}^{l_{k}} \prod_{n=1}^{7}\left(a_{n} ! b_{n} ! c_{n} !\right)^{m_{n}} C_{0}^{m_{n}\left(a_{n}+b_{n}+c_{n}\right)} \leq C_{0}^{3 k+1} k ! .
$$

Finally, we count the maximum number of terms in (5.19). When we take one derivative in $\lambda$ on $\mathcal{C}_{+}^{\lambda} f$, we get three terms by using (5.17); when we take one derivative in $z$ or $\bar{z}$ on $\mathcal{C}_{+}^{\lambda} f$, we have just one term in (5.18). Therefore,

$$
N_{k} \stackrel{\text { def }}{=} \max _{i, j} N_{i, j ; k} \leq 3^{k} .
$$

We have $\left|Q_{i, j, k-1, l}(\cdot, \cdot)\right|_{\alpha} \leq C_{1}\left|Q_{i, j, k-1, l}(\cdot, \cdot)\right|_{1}$. Taking a $\zeta$-derivative on $P_{i, j, k-1, l}^{\lambda}$. $Q_{i, j, k-1, l}^{\lambda}$ introduces at most $N_{k}$ terms of the form $P_{i^{\prime}, j^{\prime}, k, l^{\prime}}^{\lambda} \times Q_{i^{\prime}, j^{\prime}, k, l^{\prime}}^{\lambda}$. This shows 
that

$$
\begin{aligned}
\left|P_{i, j, k-1, l}(\cdot)\right|_{0}\left|Q_{i, j, k-1, l}(\cdot)\right|_{\alpha} & \leq N_{k} C_{1} \max _{i^{\prime}, j^{\prime}, l^{\prime}}\left|P_{i^{\prime}, j^{\prime}, k, l^{\prime}}(\cdot)\right|_{0}\left|Q_{i^{\prime}, j^{\prime}, k, l^{\prime}}(\cdot)\right|_{0} \\
& \leq C_{1} 3^{k} k ! C_{0}^{3 k+1} \quad(\text { by (15.21) }
\end{aligned}
$$

By (5.19), (5.14) and the above inequality, we obtain

$$
\begin{aligned}
\left|\partial_{z}^{i} \partial_{\bar{z}}^{j} \partial_{\lambda}^{k-1-i-j}\left\{\mathcal{C}_{+}^{\lambda} f^{\lambda}\left(z^{\lambda}\right)\right\}\right| & \leq N_{k-1} C_{1,0}\left|P_{i, k-1-i, l}(\cdot, \cdot)\right|_{0}\left|Q_{i, k-1-i, l}(\cdot, \cdot)\right|_{\alpha} \\
& \leq C_{1} C_{1,0} 3^{2 k-1} k ! C_{0}^{3 k+1} .
\end{aligned}
$$

Using $k ! \leq i ! j !(k-1-i-j) ! 3^{k-1} k$, we obtain the desired estimate on Taylor coefficients to show that $\mathcal{C}_{+}^{\lambda} f^{\lambda}\left(z^{\lambda}\right)$ is real analytic on $\bar{\Omega} \times[0,1]$.

It is clear that $W^{\lambda} f(z)$ is real analytic on $\Omega \times[0,1]$. We need to show that it is real analytic near $\left(z_{1}, \lambda_{1}\right) \in \bar{\Omega} \times[0,1]$ for $z_{1} \in \partial \Omega$. We use local real analytic coordinates to find a real analytic function $\varphi\left(z_{0}, z, t\right)$ defined on $U \times U \times[0,1]$ such that $\varphi\left(z_{0}, z, 0\right)=z_{0}$ and $\varphi\left(z_{0}, z, 1\right)=z$, where $U$ is an open set containing $z_{1}$. Moreover, $\varphi\left(z_{0}, z, t\right)$ is in $\Omega$ when $t \in(0,1)$ and $z_{0}, z$ are in $U \cap \bar{\Omega}$. Fix $z_{0} \in U \cap \Omega$ and vary $z \in U \cap \bar{\Omega}$. We have

$$
W^{\lambda} f\left(\Gamma^{\lambda}(z)\right)=W^{\lambda} f\left(\Gamma^{\lambda}\left(z_{0}\right)\right)+2 \operatorname{Re} \int_{0}^{1}\left\{\partial_{z}\left(\left(W^{\lambda} f\right) \circ \Gamma^{\lambda}\right)\right\}\left(\varphi\left(z_{0}, z, t\right)\right) \partial_{t} \varphi\left(z_{0}, z, t\right) d t .
$$

Since $\partial_{z^{\lambda}} W^{\lambda} f=-2 i C_{+}^{\lambda}\left(\bar{\tau}^{\lambda} f^{\lambda}\right)$ and $\partial_{\bar{z}^{\lambda}} W^{\lambda} f^{\lambda}$ are real analytic in $(z, \lambda) \in \bar{\Omega} \times[0,1]$, then $\partial_{z} W^{\lambda} f$ is real analytic in $z$ and $\lambda$ by the chain rule. Thus, the integrand in the above integral is real analytic in $\left(z_{0}, z, \lambda, t\right) \in(U \cap \bar{\Omega})^{2} \times[0,1]^{2}$. This shows that $W^{\lambda} f^{\lambda}\left(z^{\lambda}\right)$ is real analytic in $(z, \lambda) \in \bar{\Omega} \times[0,1]$.

We have seen that the kernels of $U f$ and $W f$ behave better than that of $\mathcal{C} f$ for spaces of continuous functions. In the parameter case, we have the following analogue of (3.3) and Lemma 3.2 (iii).

Proposition 5.3. Let $\Gamma^{\lambda}$ embed $\bar{\Omega}$ onto $\overline{\Omega^{\lambda}}$ with $\Gamma \in \mathcal{C}_{*}^{1, j}(\bar{\Omega})$. Suppose that $\Gamma^{\lambda}$ preserves the orientation. Assume that $f \in \mathcal{C}_{*}^{0, j}\left(\partial \Omega_{\gamma}\right)$. Then $W f \in \mathcal{C}_{*}^{0, j}\left(\bar{\Omega}_{\Gamma}\right)$ and

$$
\partial_{\lambda}^{j} W^{\lambda} f(z)=\frac{1}{\pi} \sum_{i=0}^{j}\left(\begin{array}{l}
j \\
i
\end{array}\right) \int_{\partial \Omega} \partial_{\lambda}^{i}\left(\left|\partial_{\tau} \gamma^{\lambda}(\zeta)\right| f^{\lambda}\left(\zeta^{\lambda}\right)\right) \partial_{\lambda}^{j-i}\left\{\log \left|\zeta^{\lambda}-z^{\lambda}\right|\right\} d \sigma(\zeta), z \in \bar{\Omega} .
$$

Assume further that $\partial \Omega \in \mathcal{C}^{1+\alpha}$ and $\Gamma \in \mathcal{B}_{*}^{1+\alpha, j}(\bar{\Omega})$. Then $U f \in \mathcal{C}_{*}^{0, j}\left(\bar{\Omega}_{\Gamma}\right)$ and

$$
\begin{aligned}
\partial_{\lambda}^{j} U_{+}^{\lambda} f(z)= & \frac{1}{\pi} \sum_{i=0}^{j}\left(\begin{array}{c}
j \\
i
\end{array}\right) \int_{\partial \Omega} \partial_{\lambda}^{i}\left\{f^{\lambda}\left(\zeta^{\lambda}\right)\right\} \partial_{\lambda}^{j-i}\left\{\left|\partial_{\tau} \gamma^{\lambda}(\zeta)\right| \partial_{\tau^{\lambda}} \arg \left(\zeta^{\lambda}-z^{\lambda}\right)\right\} d \sigma(\zeta) \\
& +\partial_{\lambda}^{j}\left(f^{\lambda}\left(z^{\lambda}\right)\right)
\end{aligned}
$$

holds on $\partial \Omega$. Under the norms defined by (2.1)-(2.3) and (5.11)-(5.13),

$$
\left|W_{+} f\right|_{0, j} \leq C_{1, j}^{*}|f|_{0, j},\left|W_{+}^{\mu} f-W_{+}^{\lambda} f\right|_{0, j} \leq C_{1, j}^{*}\left(\left|\Gamma^{\mu}-\Gamma^{\lambda}\right|_{1, j}\left|f^{\lambda}\right|_{0, j}+\left|f^{\mu}-f^{\lambda}\right|_{0, j}\right) .
$$

In particular, if $\partial \Omega \in \mathcal{C}^{k+1+\alpha}, \Gamma \in \mathcal{B}^{k+1+\alpha, j}(\bar{\Omega})$ and $f \in \mathcal{B}^{k+\alpha, j}\left(\partial \Omega_{\gamma}\right)$ with $k \geq j$, then $W_{+} f \in \mathcal{B}^{k+1+\alpha, j}\left(\bar{\Omega}_{\Gamma}\right)$; the same assertion holds if $\mathcal{C}$ substitutes for $\mathcal{B}$. 
Proof. We write $d \sigma^{\lambda}=a^{\lambda} d \sigma$ on $\partial \Omega$. Recall from (2.6) that $a^{\lambda}(z)=\left|\partial_{\tau} \gamma^{\lambda}(z)\right|$. Fix $z_{0} \in \partial \Omega$. For $z \in \Omega$ we have $\partial_{\lambda}^{j} U^{\lambda} f(z)=\sum\left(\begin{array}{l}j \\ k\end{array}\right) I_{k}(z)$ with

$$
I_{k}^{\lambda}(z)=\frac{1}{\pi} \int_{\partial \Omega} \partial_{\lambda}^{k}\left\{f^{\lambda}\left(\zeta^{\lambda}\right)\right\} \partial_{\lambda}^{j-k}\left\{a^{\lambda}(\zeta) \partial_{\tau_{\zeta}^{\lambda}} \arg \left(z^{\lambda}-\zeta^{\lambda}\right)\right\} d \sigma .
$$

Using (5.5) for $\gamma \in \mathcal{B}_{*}^{1+\alpha, j}$, we get for $l \leq j$

$$
\begin{gathered}
\int_{\zeta \in \partial \Omega,\left|\zeta-z_{0}\right|<\epsilon}\left|\partial_{\lambda}^{l} \partial_{\tau_{\zeta}^{\lambda}} \arg \left(z^{\lambda}-\zeta^{\lambda}\right)\right| d \sigma^{\lambda} \leq C \epsilon^{\alpha}, \quad z_{0} \in \partial \Omega, \\
\int_{\zeta \in \partial \Omega}\left|\partial_{\lambda}^{l} \partial_{\tau_{\zeta}^{\lambda}} \arg \left(z^{\lambda}-\zeta^{\lambda}\right)\right| d \sigma^{\lambda}<C, \quad z \in \mathbf{C} .
\end{gathered}
$$

Then, by analogue of (3.8), from $f \in \mathcal{C}_{*}^{0, j}, \gamma \in \mathcal{B}_{*}^{1, j}$ and (5.24) we get

$$
\begin{aligned}
& \int_{\partial \Omega}\left\{\partial_{\lambda}^{k}\left\{f^{\lambda}\left(\zeta^{\lambda}\right)\right\}-\partial_{\lambda}^{k}\left\{f^{\lambda}\left(z_{0}^{\lambda}\right)\right\}\right\} \partial_{\lambda}^{j-k}\left\{a^{\lambda}(\zeta) \partial_{\tau_{\zeta}^{\lambda}} \arg \left(z^{\lambda}-\zeta^{\lambda}\right)\right\} d \sigma(\zeta) \\
& \rightarrow \int_{\partial \Omega}\left\{\partial_{\lambda}^{k}\left\{f^{\lambda}\left(\zeta^{\lambda}\right)\right\}-\partial_{\lambda}^{k}\left\{f^{\lambda}\left(z_{0}^{\lambda}\right)\right\}\right\} \partial_{\lambda}^{j-k}\left\{a^{\lambda}(\zeta) \partial_{\tau_{\zeta}^{\lambda}} \arg \left(z_{0}^{\lambda}-\zeta^{\lambda}\right)\right\} d \sigma(\zeta)
\end{aligned}
$$

as $\partial \Omega \not \supset z \rightarrow z_{0}$, where the convergence is uniform in $\lambda$. Let $0<l \leq j$. Note that

$$
\int_{\partial \Omega} \partial_{\lambda}^{l}\left\{a^{\lambda}(\zeta) \partial_{\tau_{\zeta}^{\lambda}} \arg \left(z^{\lambda}-\zeta^{\lambda}\right)\right\} d \sigma(\zeta)=0, \quad z \notin \partial \Omega .
$$

For $z=z_{0} \in \partial \Omega$ and $\gamma \in \mathcal{C}_{*}^{1, j}$, the last integral equals

$$
\begin{aligned}
& \lim _{\epsilon \rightarrow 0} \int_{\zeta \in \partial \Omega,\left|\zeta-z_{0}\right|>\epsilon} \partial_{\lambda}^{l}\left\{a^{\lambda}(\zeta) \partial_{\tau_{\zeta}^{\lambda}} \arg \left(z_{0}^{\lambda}-\zeta^{\lambda}\right)\right\} d \sigma \\
& =\lim _{\epsilon \rightarrow 0} \partial_{\lambda}^{l}\left\{\pi-\arg \left(\gamma^{\lambda} \circ \hat{\gamma}\left(\epsilon_{2}\right)-\gamma^{\lambda} \circ \hat{\gamma}(0)\right)-\arg \left(\gamma^{\lambda} \circ \hat{\gamma}(0)-\gamma^{\lambda} \circ \hat{\gamma}\left(\epsilon_{1}\right)\right)\right\} \\
& =\lim _{\epsilon \rightarrow 0} \partial_{\lambda}^{l}\left\{\pi-\arctan \frac{\int_{0}^{1}\left(y^{\lambda} \circ \hat{\gamma}\right)^{\prime}\left(r \epsilon_{2}\right) d r}{\int_{0}^{1}\left(x^{\lambda}\right)^{\prime}\left(r \epsilon_{2}\right) d r}+\arctan \frac{\int_{0}^{1}\left(y^{\lambda}\right)^{\prime}\left(r \epsilon_{1}\right) d r}{\int_{0}^{1}\left(x^{\lambda}\right)^{\prime}\left(r \epsilon_{1}\right) d r}\right\}=0 .
\end{aligned}
$$

Here we have used $\arg (x+i y)=\arctan (y / x) \bmod \pi$ and a local $\mathcal{C}^{1}$ parameterization $\hat{\gamma}$ of $\partial \Omega$ near $z_{0}$ with $\hat{\gamma}(0)=z_{0}$. Also, $\partial \Omega$ intersects $\left\{\left|\zeta-z_{0}\right|=\epsilon\right\}$ at $\hat{\gamma}\left(\epsilon_{1}\right)$, $\hat{\gamma}\left(\epsilon_{2}\right)$ for $\epsilon$ sufficiently small. We have also assumed without loss of generality that $\partial_{\tau} x^{\lambda}\left(z_{0}\right) \neq 0$. Expanding both sides of (5.25) we get the formula for $\partial_{\lambda}^{j} U_{+}^{\lambda} f$. Combining the formula with (5.23), we see that $U_{+}^{\lambda} f\left(z_{0}^{\lambda}\right)$ is continuous in $\lambda$. Then, the uniform convergence of $U^{\lambda} f\left(z^{\lambda}\right)$ as $z \rightarrow z_{0}$ yields $U_{+} f \in \mathcal{C}_{*}^{0, j}\left(\bar{\Omega}_{\Gamma}\right)$.

Write $\partial_{\lambda}^{j} W^{\lambda} f$ as a linear combination of

$$
h_{j_{1} j_{2}}^{\lambda}(z)=\int_{\partial \Omega} \partial_{\lambda}^{j_{1}}\left\{a^{\lambda}(\zeta) f^{\lambda}\left(\zeta^{\lambda}\right)\right\} \partial_{\lambda}^{j_{2}} \log \left|z^{\lambda}-\zeta^{\lambda}\right| d \sigma, \quad j_{1}+j_{2}=j .
$$

Using (5.3) and (3.5), we obtain

$$
\left|\partial_{\lambda}^{j_{2}} \log \right| \zeta^{\lambda}-z^{\lambda}|| \leq C, j_{2}>0 ; \quad|\log | \zeta^{\lambda}-z^{\lambda}|| \leq C(|\log | t-s||+1),
$$

where $z=\hat{\gamma}(s)+h \nu(s), \zeta=\hat{\gamma}(t)$, and $\hat{\gamma}$ is a parameterization of $\partial \Omega$. We conclude easily that $h_{j_{1} j_{2}}^{\lambda}(z)$ are continuous on $\bar{\Omega} \times[0,1]$. This verifies the formula for $\partial_{\lambda}^{j} W_{+}^{\lambda} f$.

By the formulae of $\partial_{\lambda}^{j} W^{\lambda} f$, we obtain $W_{+} f \in \mathcal{C}_{*}^{0, j}\left(\bar{\Omega}_{\Gamma}\right)$ and the desired estimate for $W_{+} f$ by (5.3)-(15.4), $\int_{\partial \Omega^{\lambda}}|\log | \zeta^{\lambda}-z^{\lambda}|| d \sigma^{\lambda}<C$ and $\operatorname{dist}(z, \partial \Omega) \int_{\partial \Omega} \mid \zeta-$ $\left.z\right|^{-2} d \sigma<C$ for $z \in \Omega$. The assertion on higher-order derivatives follows from $\partial_{z} W f=-i \mathcal{C}[\bar{\tau} f]$ and Proposition 5.2 
To prepare our estimates in section 7 for integral equations with parameter, we use the rest of the section to extend Lemma 4.1 to the parameter case.

For convenience, we will use the following difference operators:

$$
\delta^{\lambda \mu} f=f^{\mu}-f^{\lambda}, \quad \delta_{t_{1} t_{2}} g=g\left(t_{2}\right)-g\left(t_{1}\right) .
$$

For clarity we will also write the above as $\delta^{\lambda \mu} f^{\bullet}$ and $\delta_{t_{1} t_{2}} g(\cdot)$, where $\cdot$ and $\bullet$ indicate the variables used in the operators. Both satisfy the product rule to the extent that

$$
\delta^{\lambda \mu}(f g)=\delta^{\lambda \mu} f g^{\nu}+f^{\nu} \delta^{\lambda \mu} g, \quad \delta_{t_{1} t_{2}}(f g)=\delta_{t_{1} t_{2}} f g\left(t_{3}\right)+f\left(t_{3}\right) \delta_{t_{1} t_{2}} g,
$$

where $\nu=\lambda$ or $\mu$ (and two $\nu$ 's are different), and $t_{3}=t_{1}$ or $t_{2}$. For $\gamma \in$ $\mathcal{B}^{k+1+\alpha, j}(\partial \Omega)$, with the above notation we have

$$
\begin{aligned}
& K_{k-j, j}^{\lambda}\left(\zeta^{\lambda}, z^{\lambda}\right):=\partial_{\tau_{z}^{\lambda}}^{k-j} \partial_{\lambda}^{j} \partial_{\tau_{z}^{\lambda}}\left\{\arg \left(\zeta^{\lambda}-z^{\lambda}\right)\right\}, \quad \zeta^{\lambda}, z^{\lambda} \in \partial \Omega^{\lambda}, \\
& \delta^{\lambda \mu} K_{k-j, j}(\zeta, z)=K_{k-j, j}^{\mu}\left(\zeta^{\mu}, z^{\mu}\right)-K_{k-j, j}^{\lambda}\left(\zeta^{\lambda}, z^{\lambda}\right), \quad \zeta, z \in \partial \Omega .
\end{aligned}
$$

Lemma 5.4. Let $\gamma^{\lambda}$ embed $\partial \Omega$ onto $\partial \Omega^{\lambda}$ with $\gamma \in \mathcal{C}^{k+1+\alpha, j}(\partial \Omega)$ and $k \geq j$. Then on $\partial \Omega^{\lambda} \times \partial \Omega^{\lambda}$ and off its diagonal,

$$
\begin{gathered}
\left|K_{k-j, j}^{\lambda}\left(\zeta^{\lambda}, z^{\lambda}\right)\right| \leq C_{k+1+\alpha, j}|\zeta-z|^{\alpha-1} \\
\left|K_{k-j, j}^{\lambda}\left(\zeta^{\lambda}, z_{2}^{\lambda}\right)-K_{k-j, j}^{\lambda}\left(\zeta^{\lambda}, z_{1}^{\lambda}\right)\right| \leq C_{k+1+\alpha, j} \frac{\left|z_{2}-z_{1}\right|^{\alpha}}{\left|\zeta-z_{1}\right|} \\
\left|\delta^{\lambda \mu} K_{k-j, j}(\zeta, z)\right| \leq C_{k+1+\alpha, j}\left\|\gamma^{\mu}-\gamma^{\lambda}\right\|_{k+1+\alpha, j}|\zeta-z|^{\alpha-1} \\
\left|\delta_{z_{1} z_{2}} \delta^{\lambda \mu} K_{k-j, j}(\zeta, \cdot)\right| \leq C_{k+1+\alpha, j}\left\|\gamma^{\mu}-\gamma^{\lambda}\right\|_{k+1+\alpha, j} \frac{\left|z_{2}-z_{1}\right|^{\alpha}}{\left|\zeta-z_{1}\right|} \\
\left|\delta^{\lambda \mu} K_{0, j}\left(z_{2}, \zeta\right)-\delta^{\lambda \mu} K_{0, j}\left(z_{1}, \zeta\right)\right| \leq C_{1+\alpha, j}^{*}\left\|\gamma^{\mu}-\gamma^{\lambda}\right\|_{1+\alpha, j} \frac{\left|z_{2}-z_{1}\right|}{\left|\zeta-z_{1}\right|^{2-\alpha}}
\end{gathered}
$$

where (5.27), (5.29) and (5.30) are for $\left|\zeta-z_{1}\right|>2\left|z_{2}-z_{1}\right|$.

Proof. It suffices to verify (5.26)-(5.29) for $\zeta, z_{1}, z_{2}, z$ near a point $w \in \partial \Omega$ at which $\left|\partial_{\tau_{w}} x^{\lambda}\right| \neq 0$. We may assume that $w=0$ and $\partial \Omega$ contains $(-1,1)+i 0$. We may assume that $\left\|\gamma^{\mu}-\gamma^{\lambda}\right\|_{1,0}$ is sufficiently small; otherwise, (5.28)-(5.29) follow from (5.26)-(5.27). We may further assume that $\partial \Omega^{\lambda}$ is embedded through $\gamma^{\lambda}(t)$ such that, for $|t| \leq 1,\left(x^{\lambda}\right)^{\prime}>1 / C$ and $\left|\left(x^{\lambda}(s)-x^{\lambda}(t)\right)^{-1}\right| \leq C|s-t|^{-1}$. In the following, we assume that $s, t, t_{1}$ and $t_{2}$ are in $(-1,1)$. Define $q^{\lambda}(s, t)=$ $\left(y^{\lambda}(s)-y^{\lambda}(t)\right) /\left(x^{\lambda}(s)-x^{\lambda}(t)\right)$ and

$$
\hat{K}_{k-j, j}^{\lambda}(s, t)=\partial_{\lambda}^{j} \partial_{t}^{k+1-j} q^{\lambda}(s, t), \quad \delta^{\lambda \mu} \hat{K}_{k-j, j}(s, t)=\hat{K}_{k-j, j}^{\mu}(s, t)-\hat{K}_{k-j, j}^{\lambda}(s, t) .
$$

By (2.7), we have $\left(\partial_{\tau^{\lambda}} u^{\lambda}\right)\left(\gamma^{\lambda}(t)\right)=\left|\partial_{t} \gamma^{\lambda}\right|^{-1} \partial_{t}\left(u^{\lambda}\left(\gamma^{\lambda}\right)\right)$. Hence

$$
K_{k-j, j}^{\lambda}\left(\gamma^{\lambda}(s), \gamma^{\lambda}(t)\right)=\sum_{j^{\prime} \leq j, k^{\prime} \leq k} Q_{j^{\prime} k^{\prime}}^{\lambda}(t) \hat{K}_{k^{\prime}-j^{\prime}, j^{\prime}}^{\lambda}(s, t) .
$$

Here $Q_{j^{\prime} k^{\prime}}^{\lambda}$ are $C^{\infty}$ functions in $\left|\partial_{t} \gamma^{\lambda}\right|^{-1}, \partial_{t} \gamma^{\lambda}, \ldots, \partial_{t}^{k+1} \gamma^{\lambda}$, and

$$
q^{\lambda}(s, t)=\frac{\int_{0}^{1}\left(y^{\lambda}\right)^{\prime}(s+r(t-s)) d r}{\int_{0}^{1}\left(x^{\lambda}\right)^{\prime}(s+r(t-s)) d r} .
$$


We have for $Q^{\lambda}=Q_{j^{\prime} k^{\prime}}^{\lambda}$,

$$
\begin{gathered}
\left|Q^{\lambda}(t)\right| \leq C_{k+1, j}, \quad\left|Q^{\lambda}\left(t_{2}\right)-Q^{\lambda}\left(t_{1}\right)\right| \leq C_{k+1+\alpha, j}\left|t_{2}-t_{1}\right|^{\lambda}, \\
\left|\left(Q^{\mu}-Q^{\lambda}\right)\left(t_{2}\right)-\left(Q^{\mu}-Q^{\lambda}\right)\left(t_{1}\right)\right| \leq C_{k+1+\alpha, j}\left\|\gamma^{\mu}-\gamma^{\lambda}\right\|_{k+1+\alpha, j}\left|t_{2}-t_{1}\right|^{\lambda} .
\end{gathered}
$$

Therefore, to show (5.26)-(5.30), it suffices to verify them when $K^{\lambda}\left(\zeta^{\lambda}, z^{\lambda}\right), \zeta$, and $z$ are replaced by $\hat{K}^{\lambda}(s, t), s$, and $t$, respectively.

Recall that $\hat{R}_{k} f(s, t)=\int_{0}^{1}\left\{f^{(k)}(t+r(s-t))-f^{(k)}(t)\right\} d r$. We apply formula (4.5) and obtain for $\left(x^{\lambda}(s)-x^{\lambda}(t)\right) q^{\lambda}(s, t)=y^{\lambda}(s)-y^{\lambda}(t)$,

$$
\begin{aligned}
\left(x^{\lambda}(s)\right. & \left.-x^{\lambda}(t)\right)^{k+2-j} \partial_{t}^{k+2-j} q^{\lambda}(s, t) \\
& =(s-t)^{k+1-j}\left\{P_{0}^{\lambda} \hat{R}_{k+1-j} y^{\lambda}(s, t)+\sum_{i=1}^{k+1-j} P_{i}^{\lambda} \hat{R}_{i} x^{\lambda}(s, t)\right\},
\end{aligned}
$$

where $P_{i}^{\lambda}(s, t)$ are polynomials in $s-t, \partial_{t}\left(x^{\lambda}, y^{\lambda}\right), \ldots, \partial_{t}^{k+1-j}\left(x^{\lambda}, y^{\lambda}\right), \partial_{s}^{k+1-j} \gamma^{\lambda}$, and $x^{\lambda}(s)-x^{\lambda}(t)$. Hence $\partial_{\lambda}^{j} \partial_{t}^{k+1-j} q^{\lambda}(s, t)$ is a linear combination of $L^{\lambda}(s, t)$ of the form

$$
(s-t)^{k+1-j} \frac{\partial_{\lambda}^{j_{1}}\left(x^{\lambda}(s)-x^{\lambda}(t)\right) \cdots \partial_{\lambda}^{j_{a}}\left(x^{\lambda}(s)-x^{\lambda}(t)\right)}{\left(x^{\lambda}(s)-x^{\lambda}(t)\right)^{k+2-j+a}} \partial_{\lambda}^{j_{b}} P_{i}^{\lambda} \partial_{\lambda}^{j_{c}} \hat{R}_{i}\left(x^{\lambda}, y^{\lambda}\right)(s, t) .
$$

Here $j_{1}+\cdots+j_{a}+j_{b}+j_{c}=j$ and $i+j \leq k+1$. Assume that $j^{\prime} \leq j$. We first bound each term in $L^{\lambda}(s, t)$ as follows:

$$
\begin{gathered}
\left|\left(\partial_{\lambda}^{j} \gamma^{\lambda}(s)-\partial_{\lambda}^{j} \gamma^{\lambda}(t)\right)\right| \leq C|s-t|, \quad\left|\left(x^{\lambda}(s)-x^{\lambda}(t)\right)^{-1}\right| \leq C|s-t|^{-1} \\
\left|\partial_{\lambda}^{j} \hat{R}_{i} \gamma^{\lambda}(s, t)\right| \leq C|s-t|^{\alpha}, \quad\left|\partial_{\lambda}^{j} P_{i}^{\lambda}(s, t)\right| \leq C, \quad i+j \leq k+1 .
\end{gathered}
$$

By (5.31)-(5.32) we get $\left|L^{\lambda}(s, t)\right| \leq C|s-t|^{\alpha-1}$, which gives us (5.26). We now assume that $\left|s-t_{2}\right| \geq 2\left|t_{2}-t_{1}\right|$. Then

$$
\begin{gathered}
\left|\left(s-t_{2}\right)-\left(x-t_{1}\right)\right| \leq\left|s-t_{1}\right|^{1-\alpha}\left|t_{2}-t_{1}\right|^{\alpha}, \\
\left|\partial_{\lambda}^{j} x^{\lambda}\left(t_{2}\right)-\partial_{\lambda}^{j} x^{\lambda}\left(t_{1}\right)\right| \leq C\left|s-t_{1}\right|^{1-\alpha}\left|t_{2}-t_{1}\right|^{\alpha}, \\
\left|\left(x^{\lambda}\left(s, t_{2}\right)\right)^{-1}-\left(x^{\lambda}\left(s, t_{1}\right)\right)^{-1}\right| \leq C\left|s-t_{1}\right|^{-1-\alpha}\left|t_{2}-t_{1}\right|^{\alpha}, \\
\left|\partial_{\lambda}^{j}\left(P_{i}^{\lambda}, \hat{R}_{i} \gamma^{\lambda}\right)\left(s, t_{2}\right)-\partial_{\lambda}^{j}\left(P_{i}^{\lambda}, \hat{R}_{i} \gamma^{\lambda}\right)\left(s, t_{1}\right)\right| \leq C|| \gamma\left|\|_{k+1+\alpha, j}\right| t_{2}-\left.t_{1}\right|^{\alpha} .
\end{gathered}
$$

Here $i+j \leq k+1$. Comparing the exponents in (5.31)-(5.32) with the ones in (5.33)(5.36), and using the exponent in (5.26), we obtain (5.27) by a simple computation.

Applying $\delta^{\lambda \mu}$ to each term in $L^{\lambda}(s, t)$, we get

$$
\begin{gathered}
\left|\delta^{\lambda \mu}\left(\partial_{\bullet}^{j} x^{\bullet}(s)-\partial_{\bullet}^{j} x^{\bullet}(t)\right)\right| \leq C\left\|\gamma^{\mu}-\gamma^{\lambda}\right\|_{k+1, j}|s-t|, \\
\left|\delta^{\lambda \mu}\left((x(s)-x(t))^{-1}\right)\right| \leq C\left\|\gamma^{\mu}-\gamma^{\lambda}\right\|_{k+1, j}|s-t|, \\
\left|\delta^{\lambda \mu}\left(\partial_{\bullet}^{j} P_{i}^{\bullet}(s, t)\right)\right| \leq C\left\|\gamma^{\mu}-\gamma^{\lambda}\right\|_{k+1, j}, \\
\left|\delta^{\lambda \mu}\left(\left(\partial_{\bullet}^{j} \hat{R}_{i}^{\bullet} \gamma\right)(s, t)\right)\right| \leq C\left\|\gamma^{\mu}-\gamma^{\lambda}\right\|_{k+1+\alpha, j}|s-t|^{\alpha}, \quad i+j \leq k+1 .
\end{gathered}
$$

We see that (5.37)-(5.40) and (5.31)-(5.32) differ by a factor $\left\|\gamma^{\mu}-\gamma^{\lambda}\right\|_{k+1+\alpha, j}$, as (5.26) and (5.28) do. A simple computation gives us (5.28). We have

$$
\delta^{\lambda \mu}(x(s)-x(t))^{-1}=\left(x^{\mu}(t)-x^{\mu}(s)\right)^{-1}\left(x^{\lambda}(s)-x^{\lambda}(t)\right)^{-1} \delta^{\lambda \mu}(x(s)-x(t)) .
$$


Note that $\delta_{t_{1} t_{2}}$ and $\delta^{\lambda \mu}$ commute. Assume that $\left|s-t_{1}\right|>2\left|t_{2}-t_{1}\right|$. Then

$$
\begin{aligned}
& \left|\delta_{t_{1} t_{2}} \delta^{\lambda \mu}(x(s)-x(\cdot))^{-1}\right| \leq 2 C\left|t_{2}-t_{1}\right|\left|s-t_{1}\right|^{-2}\left|\gamma^{\mu}-\gamma^{\lambda}\right|_{0}\left|s-t_{1}\right| \\
& \quad+C\left|s-t_{1}\right|^{-2}\left|\gamma^{\mu}-\gamma^{\lambda}\right|_{1}\left|t_{2}-t_{1}\right| \leq 3 C\left|\gamma^{\mu}-\gamma^{\lambda}\right|_{1}\left|s-t_{1}\right|^{-1-\alpha}\left|t_{2}-t_{1}\right|^{\alpha} .
\end{aligned}
$$

Therefore,

$$
\begin{aligned}
& \left|\delta^{\lambda \mu} \delta_{t_{1} t_{2}}\left(\partial_{\bullet}^{j} \gamma^{\bullet}(s)-\partial_{\bullet}^{j} \gamma^{\bullet}(\cdot)\right)\right| \leq C\left|\gamma^{\mu}-\gamma^{\lambda}\right|_{1, j}\left|s-t_{1}\right|^{1-\alpha}\left|t_{2}-t_{1}\right|^{\alpha}, \\
& \left|\delta^{\lambda \mu} \delta_{t_{1} t_{2}}(x(s, \cdot))^{-1}\right| \leq C\left|\gamma^{\mu}-\gamma^{\lambda}\right|_{1,0}\left|s-t_{1}\right|^{-1-\alpha}\left|t_{2}-t_{1}\right|^{\alpha}, \\
& \left|\delta^{\lambda \mu} \delta_{t_{1} t_{2}} \partial_{\bullet}^{j}\left(P_{i}^{\bullet}, \hat{R}_{i} \gamma^{\bullet}\right)(s, \cdot)\right| \leq C\left\|\gamma^{\mu}-\gamma^{\lambda}\right\|_{k+1+\alpha, j}\left|t_{2}-t_{1}\right|^{\alpha} .
\end{aligned}
$$

Here $i+j \leq k+1$. Comparing (5.34)-(5.36) with (5.31)-(5.32) and (5.41)-(5.43) with (5.33)-(5.36), we see that applying $\delta^{\lambda \mu}$ introduces a factor $\left|\gamma^{\mu}-\gamma^{\lambda}\right|_{k+1+\alpha, j}$, as shown in (5.27) and (5.29). A simple computation gives us (5.29).

To verify (5.30), we start with

$$
\hat{K}^{\lambda}(t, s)=\operatorname{Im} \frac{\overline{\partial_{s} \gamma^{\lambda}} \int_{t}^{s}\left(\partial_{r} \gamma^{\lambda}-\partial_{s} \gamma^{\lambda}\right) d r}{\left|\partial_{s} \gamma^{\lambda}\right|\left|\gamma^{\lambda}(s)-\gamma^{\lambda}(t)\right|^{2}} .
$$

Then $\partial_{\lambda}^{j} \hat{K}^{\lambda}(t, s)$ is a linear combination of $J^{\lambda}(s, t)=A_{1}^{\lambda}(s) A_{2}^{\lambda}(s, t) A_{3}^{\lambda}(s, t)$ with

$$
\begin{gathered}
A_{1}^{\lambda}(s)=\partial_{\lambda}^{j_{1}}\left(\left|\partial_{s} \gamma^{\lambda}\right|^{-1} \overline{\partial_{s} \gamma^{\lambda}}\right), \quad A_{2}^{\lambda}(s, t)=\partial_{\lambda}^{j_{2}}\left(\left|\gamma^{\lambda}(s)-\gamma^{\lambda}(t)\right|^{-2}\right), \\
A_{3}(s, t)=\partial_{\lambda}^{j_{3}} \int_{t}^{s}\left(\partial_{r} \gamma^{\lambda}-\partial_{s} \gamma^{\lambda}\right) d r
\end{gathered}
$$

and $j_{1}+j_{2}+j_{3}=j$. Then $\left|A_{1}^{\lambda}\right| \leq C$ and

$$
\left|A_{2}^{\lambda}(s, t)\right| \leq C|s-t|^{-2},\left|A_{3}^{\lambda}(s, t)\right| \leq C|s-t|^{1+\alpha},\left|J^{\lambda}(s, t)\right| \leq C|s-t|^{\alpha-1} .
$$

Assume that $\left|s-t_{1}\right| \geq 2\left|t_{2}-t_{1}\right|$. As in the proof of (4.2), we write

$$
\begin{aligned}
\int_{t_{2}}^{s}\left(\partial_{r} \gamma^{\lambda}-\partial_{s} \gamma^{\lambda}\right) d r-\int_{t_{1}}^{s}\left(\partial_{r} \gamma^{\lambda}-\partial_{s} \gamma^{\lambda}\right) d r \\
\quad=\int_{t_{2}}^{t_{1}}\left(\partial_{r} \gamma-\partial_{t_{1}} \gamma\right) d r-\int_{t_{2}}^{t_{1}}\left(\partial_{t_{1}} \gamma^{\lambda}-\partial_{s} \gamma^{\lambda}\right) d r
\end{aligned}
$$

Applying $\partial_{\lambda}^{j}$ and then $\delta^{\lambda \mu}$ to the above, we get

$$
\begin{aligned}
& \left|A_{3}\left(s, t_{2}\right)-A_{3}\left(s, t_{1}\right)\right| \leq C\left|\gamma^{\lambda}\right|_{1+\alpha, j}\left|t_{2}-t_{1}\right|\left|s-t_{1}\right|^{\alpha}, \\
& \left|\delta^{\lambda \mu} \delta_{t_{1} t_{2}} A_{3}(s, \cdot)\right| \leq C\left|\gamma^{\mu}-\gamma^{\lambda}\right|_{1+\alpha, j}\left|t_{2}-t_{1}\right|\left|s-t_{1}\right|^{\alpha} .
\end{aligned}
$$

We can also verify that

$$
\begin{gathered}
\left|A_{2}^{\lambda}(s, t)\right| \leq C|s-t|^{-2}, \quad\left|\delta^{\lambda \mu} A_{2}(s, t)\right| \leq C\left|\gamma^{\mu}-\gamma^{\lambda}\right|_{1, j}|s-t|^{-2} \\
\left|\delta_{t_{1} t_{2}} A_{2}^{\lambda}(s, \cdot)\right| \leq C \frac{\left|t_{2}-t_{1}\right|^{\alpha}}{|s-t|^{1+\alpha}}, \quad\left|\delta_{t_{1} t_{2}} \delta^{\lambda \mu} A_{2}(s, \cdot)\right| \leq C\left|\gamma^{\mu}-\gamma^{\lambda}\right|_{1, j} \frac{\left|t_{2}-t_{1}\right|^{\alpha}}{|s-t|^{1+\alpha}} .
\end{gathered}
$$

By a simple computation, we get (5.30).

Define $\mathcal{K}_{k-j, j}^{\lambda} \varphi\left(z^{\lambda}\right)=\int_{\partial \Omega^{\lambda}} \varphi^{\lambda}\left(\zeta^{\lambda}\right) K_{k-j, j}^{\lambda}\left(z^{\lambda}, \zeta^{\lambda}\right) d \sigma^{\lambda}$ and

$$
\left(\mathcal{K}_{k-j, j}^{\lambda}\right)^{*} \varphi\left(z^{\lambda}\right)=\int_{\partial \Omega^{\lambda}} \varphi^{\lambda}\left(\zeta^{\lambda}\right) K_{k-j, j}^{\lambda}\left(\zeta^{\lambda}, z^{\lambda}\right) d \sigma^{\lambda} .
$$


We will also denote $\mathcal{K}^{*} \varphi=\left\{\left(\mathcal{K}^{\lambda}\right)^{*} \varphi\right\}, \mathcal{K}_{k-j, j}^{*} \varphi=\left\{\left(\mathcal{K}_{k-j, j}^{\lambda}\right)^{*} \varphi\right\}$ and

$$
\delta^{\lambda \mu} \mathcal{K}_{k-j, j}^{*} \varphi(\zeta, z)=\left(\mathcal{K}_{k-j, j}^{\mu}\right)^{*}\left(\zeta^{\mu}, z^{\mu}\right)-\left(\mathcal{K}_{k-j, j}^{\lambda}\right)^{*}\left(\zeta^{\lambda}, z^{\lambda}\right), \quad \zeta, z \in \partial \Omega .
$$

The reader is referred to (2.1)-(2.3) and (5.11)-(15.13) for the definition of norms.

Proposition 5.5. Let $k \geq j$ and $0 \leq \beta \leq \alpha$. Let $\alpha^{\prime}=\alpha$ for $\beta>0$ and $\alpha^{\prime}<\alpha$ for $\beta=0$. Let $\Omega$ be a bounded domain in $\mathbf{C}$ with $\partial \Omega \in \mathcal{C}^{k+1+\alpha}$. Let $\gamma^{\lambda}$ embed $\partial \Omega$ onto $\partial \Omega^{\lambda}$ with $\gamma \in \mathcal{B}^{k+1+\alpha, j}(\partial \Omega)$. Suppose that $k \geq j$ and $k+1 \geq l \geq j$. Then

$$
\begin{gathered}
\left|\mathcal{K}_{0 j^{\prime}} \varphi\right|_{\alpha, j-j^{\prime}} \leq C_{1+\alpha, j}^{*}|\varphi|_{0, j-j^{\prime}}, \quad\left|\mathcal{K}_{0 j^{\prime}}^{*} \varphi\right|_{\alpha^{\prime}, j-j^{\prime}} \leq C_{1+\alpha, j}^{*}|\varphi|_{0, j-j^{\prime}}, \\
\left|\delta^{\lambda \mu} \mathcal{K}_{0 j^{\prime}} \varphi\right|_{\alpha, j-j^{\prime}} \leq C_{j+1+\alpha, j}\left(\left|\delta^{\lambda \mu} \varphi\right|_{0, j-j^{\prime}}+\left|\delta^{\lambda \mu} \gamma\right|_{j+1+\alpha, j}\right) \\
\left|\mathcal{K}_{i j^{\prime}}^{*} \varphi\right|_{k-i+\alpha^{\prime}, j-j^{\prime}} \leq C_{k+1+\alpha, j}|\varphi|_{j-j^{\prime}+\beta, j-j^{\prime}}, \\
\left|\delta^{\lambda \mu} \mathcal{K}_{i j^{\prime}}^{*} \varphi\right|_{k-i+\alpha^{\prime}, j-j^{\prime}} \leq C_{k+1+\alpha, j}\left(\left|\delta^{\lambda \mu} \varphi\right|_{j-j^{\prime}+\beta, j-j^{\prime}}+\left\|\delta^{\lambda \mu} \gamma\right\|_{k+1+\alpha^{\prime}, j}\right), \\
\left|\mathcal{K}_{i j^{\prime}} \varphi\right|_{l-i+\alpha, j-j^{\prime}} \leq C_{k+1+\alpha, j}|\varphi|_{j-j^{\prime}+1+\beta, j-j^{\prime}}, \\
\left|\delta^{\lambda \mu} \mathcal{K}_{i j^{\prime}} \varphi\right|_{l-i+\alpha^{\prime}, j-j^{\prime}} \leq C_{k+1+\alpha, j}\left(\left|\delta^{\lambda \mu} \varphi\right|_{j-j^{\prime}+1+\beta, j-j^{\prime}}+\left\|\delta^{\lambda \mu} \gamma\right\|_{k+1+\alpha, j}\right) .
\end{gathered}
$$

Proof. Recall from (2.6) that $a^{\lambda}(\zeta)=\left|\partial_{\tau_{\zeta}} \gamma^{\lambda}\right|$ and $d \sigma^{\lambda}\left(\zeta^{\lambda}\right)=a^{\lambda}(\zeta) d \sigma(\zeta)$ on $\partial \Omega$. Since $\left|\partial_{\lambda}^{j} K^{\lambda}\left(\zeta^{\lambda}, z^{\lambda}\right)\right| \leq C|\zeta-z|^{\alpha-1}$, by the mean value theorem we can change the order of differentiation and integration in $\partial_{\lambda}^{j-j^{\prime}} \mathcal{K}_{0 j^{\prime}}^{\lambda} \varphi$. The latter is then a linear combination of

$$
\int_{\partial \Omega}\left(a^{\lambda}(\zeta)\right)^{-1} \partial_{\lambda}^{j_{1}}\left(a^{\lambda}(\zeta) \varphi^{\lambda}\left(\zeta^{\lambda}\right)\right) \partial_{\lambda}^{j_{2}} K_{0 j^{\prime}}^{\lambda}\left(z^{\lambda}, \zeta^{\lambda}\right) d \sigma^{\lambda}, \quad j_{1}+j_{2}=j-j^{\prime} .
$$

By replacing $\left(a^{\lambda}(\zeta)\right)^{-1} \partial_{\lambda}^{j_{1}}\left(a^{\lambda}(\zeta) \varphi^{\lambda}\left(\zeta^{\lambda}\right)\right)$ with $\varphi^{\lambda}\left(\zeta^{\lambda}\right)$, it suffices to verify (5.44) when $j^{\prime}=j$. Analogously, we only need to verify (5.44)-(5.49) for $j^{\prime}=j$.

We have $\left|\mathcal{K}_{0, j}^{\lambda} \varphi\left(z^{\lambda}\right)\right| \leq C\left|\varphi^{\lambda}\right|_{0} \int_{\partial \Omega}|\zeta-z|^{\alpha-1} d \sigma \leq C^{\prime}\left|\varphi^{\lambda}\right|_{0}$, and by (4.1)-(4.2),

$$
\begin{aligned}
& \left|\int_{\partial \Omega} \varphi^{\lambda}\left(\zeta^{\lambda}\right)\left(K_{0, j}^{\lambda}\left(z_{2}^{\lambda}, \zeta^{\lambda}\right)-K_{0, j}^{\lambda}\left(z_{1}^{\lambda}, \zeta^{\lambda}\right)\right) d \sigma^{\lambda}\right| \\
& \quad \leq C\left|a^{\lambda} \varphi^{\lambda}\right|_{0}\left\{\int_{\left|\zeta-z_{1}\right|<3\left|z_{2}-z_{1}\right|} 2\left|\zeta-z_{1}\right|^{\alpha-1} d \sigma(\zeta)\right. \\
& \left.\quad+\int_{\left|\zeta-z_{1}\right|>\left|z_{2}-z_{1}\right|}\left|z_{2}-z_{1}\right|\left|\zeta-z_{1}\right|^{2-\alpha} d \sigma(\zeta)\right\} \leq C^{\prime}\left|\varphi^{\lambda}\right|_{0}\left|z_{2}-z_{1}\right|^{\alpha}
\end{aligned}
$$

which gives us (5.44). We have

$$
\begin{aligned}
\delta^{\lambda \mu} \mathcal{K}_{0, j} \varphi(z)= & \int_{\partial \Omega} \delta^{\lambda \mu}(a(\zeta) \varphi(\zeta)) K_{0, j}^{\mu}\left(z^{\mu}, \zeta^{\mu}\right) d \sigma \\
& +\int_{\partial \Omega} a^{\lambda}\left(\zeta^{\lambda}\right) \varphi^{\lambda}\left(\zeta^{\lambda}\right) \delta^{\lambda \mu} K_{0, j}(z, \zeta) d \sigma \stackrel{\text { def }}{=} I_{1}(z)+I_{2}(z) .
\end{aligned}
$$

Also, $\left|I_{1}(z)\right| \leq C\left(\left|\varphi^{\mu}-\varphi^{\lambda}\right|_{0}+\left|\gamma^{\mu}-\gamma^{\lambda}\right|_{1}\right)$ by (5.26). By analogue of (5.50), we get $\left|I_{1}\right|_{\alpha} \leq C\left(\left|\varphi^{\mu}-\varphi^{\lambda}\right|_{0}+\left|\gamma^{\mu}-\gamma^{\lambda}\right|_{1}\right)$. Using (5.28) and (5.30) we get $\left|I_{2}\right|_{\alpha, 0} \leq$ $C\left|\varphi^{\lambda}\right|_{0}\left|\delta^{\lambda \mu} \gamma\right|_{1+\alpha, 0}$. This shows (5.45).

By (2.7), $\left(K_{i, j}^{\lambda}\right)^{*} \varphi$ satisfies (5.46)-(5.47) if and only if $\partial_{\tau^{\lambda}}^{k-i-j}\left(K_{i, j}^{\lambda}\right)^{*} \varphi$ satisfies estimates (5.46)-(5.47) (with $i=k, j^{\prime}=j$ ). By (4.7), $\left(K_{k-j, j}^{\lambda}\right)^{*} \varphi=\partial_{\tau^{\lambda}}^{k-i-j}\left(K_{i, j}^{\lambda}\right)^{*} \varphi$. 
(The proof of (4.7) is still valid when (5.26) substitutes for (4.1).) Hence, we have reduced (5.46) - (5.47) to the case where $i=k-j$ and $j^{\prime}=j$. Using $\left|K_{k-j, j}^{\lambda}\left(\zeta^{\lambda}, z^{\lambda}\right)\right| \leq$ $C|\zeta-z|^{\alpha-1}$, we obtain $\left|\left(\mathcal{K}_{k-j, j}^{\lambda}\right)^{*} \varphi\right|_{0} \leq C|\varphi|_{0}$. For the Hölder norm, we recover a loss of regularity in Kellogg's arguments [7] by decomposing

$$
\begin{aligned}
& \left(\mathcal{K}_{k-j, j}^{\lambda}\right)^{*} \varphi\left(z_{2}\right)-\left(\mathcal{K}_{k-j, j}^{\lambda}\right)^{*} \varphi\left(z_{1}\right) \\
& =\varphi^{\lambda}\left(z_{1}^{\lambda}\right) \int_{\partial \Omega}\left\{K_{k-j, j}^{\lambda}\left(\zeta^{\lambda}, z_{2}^{\lambda}\right)-K_{k-j, j}^{\lambda}\left(\zeta^{\lambda}, z_{1}^{\lambda}\right)\right\} d \sigma^{\lambda}\left(\zeta^{\lambda}\right) \\
& \quad+\int_{\partial \Omega}\left(\varphi^{\lambda}\left(\zeta^{\lambda}\right)-\varphi^{\lambda}\left(z_{1}^{\lambda}\right)\right)\left\{K_{k-j, j}^{\lambda}\left(\zeta^{\lambda}, z_{2}^{\lambda}\right)-K_{k-j, j}^{\lambda}\left(\zeta^{\lambda}, z_{1}^{\lambda}\right)\right\} d \sigma^{\lambda}\left(\zeta^{\lambda}\right) .
\end{aligned}
$$

The first integral equals $\left(\mathcal{K}_{k-j, j}^{\lambda}\right)^{*} 1\left(z_{2}\right)-\left(\mathcal{K}_{k-j, j}^{\lambda}\right)^{*} 1\left(z_{1}\right)$. The second integral is bounded by $C\left|\varphi^{\lambda}\right|_{\beta}$ times

$$
\int_{\left|\zeta-z_{1}\right| \leq 3\left|z_{2}-z_{1}\right|}\left|\zeta-z_{1}\right|^{\alpha-1} d \sigma(\zeta)+\int_{\left|\zeta-z_{1}\right|>2\left|z_{2}-z_{1}\right|} \frac{\left|z_{2}-z_{1}\right|^{\alpha}}{\left|\zeta-z_{1}\right|^{1-\beta}} d \sigma(\zeta) .
$$

Here the sum does not exceed $C\left|z_{2}-z_{1}\right|^{\alpha}$ when $\beta>0$. If $\beta=0$, it does not exceed $C_{\alpha^{\prime}}\left|z_{2}-z_{1}\right|^{\alpha^{\prime}}$ for any $\alpha^{\prime}<\alpha$. We have

$$
\begin{aligned}
\left(\mathcal{K}_{k-j, j}^{\lambda}\right)^{*} 1(z) & =\int_{\partial \Omega} a^{\lambda}(\zeta) \partial_{\tau_{z}^{\lambda}}^{k-j} \partial_{\lambda}^{j}\left\{\mathcal{K}^{\lambda}\left(\zeta^{\lambda}, z^{\lambda}\right)\right\} d \sigma(\zeta) \\
& =\partial_{\tau_{z}^{\lambda}}^{k-j} \int_{\partial \Omega} a^{\lambda}(\zeta) \partial_{\lambda}^{j}\left\{\mathcal{K}^{\lambda}\left(\zeta^{\lambda}, z^{\lambda}\right)\right\} d \sigma(\zeta) \\
& =\sum_{l \leq j} C_{j l} \partial_{\tau_{z}^{\lambda}}^{k-j} \partial_{\lambda}^{j-l} \int_{\partial \Omega} \partial_{\lambda}^{l} a^{\lambda}(\zeta) \mathcal{K}^{\lambda}\left(\zeta^{\lambda}, z^{\lambda}\right) d \sigma(\zeta) .
\end{aligned}
$$

By Lemma 4.3 and Proposition 5.2, we get for $b_{l}^{\lambda}=\left(a^{\lambda}\right)^{-1} \partial_{\lambda}^{l} a^{\lambda}$

$$
\left|\left(\mathcal{K}^{\lambda}\right)^{*} b_{l}\right|_{k-j+\alpha, j-l} \leq C, \quad\left|\left(\mathcal{K}^{\mu}\right)^{*} b_{l}-\left(\mathcal{K}^{\lambda}\right)^{*} b_{l}\right|_{k-j+\alpha, j-l} \leq C\left\|\gamma^{\mu}-\gamma^{\lambda}\right\|_{k+1+\alpha, j} .
$$

We have verified (5.46). We have

$$
\begin{aligned}
\delta^{\lambda \mu}\left(\mathcal{K}_{k-j, j}\right)^{*} \varphi(z)= & \int_{\partial \Omega} \delta^{\lambda \mu}(a(\zeta) \varphi(\zeta)) K_{k-j, j}^{\mu}\left(\zeta^{\mu}, z^{\mu}\right) d \sigma \\
& +\int_{\partial \Omega} a^{\lambda}(\zeta) \varphi^{\lambda}\left(\zeta^{\lambda}\right) \delta^{\lambda \mu} K_{k-j, j}^{*}(\zeta, z) d \sigma .
\end{aligned}
$$

By analogue of estimation for $\left(\mathcal{K}_{k-j, j}^{\lambda}\right)^{*} \varphi$, we obtain (5.47) by (5.27) and (5.29). Finally, we obtain (5.48)-(5.49) by (5.44)-(5.47), and $\partial_{\tau^{\lambda}} \mathcal{K}^{\lambda} \varphi=-\left(\mathcal{K}^{\lambda}\right)^{*}\left(\partial_{\tau^{\lambda}} \varphi^{\lambda}\right)$.

\section{Null SPACES of $I \pm \mathcal{K}$ AND $I \pm \mathcal{K}^{*}$}

In this section, we describe results on integral equations for the Dirichlet and Neumann problems. Lacking a reference to the precise regularity in derivatives on solutions to the two problems, we derive some details. The estimates will be used in arguments for the parameter case in section 7 . As mentioned in section 1 . we reduce the $\mathcal{C}^{1}$ regularity of solutions, which is an important step in Kellogg's proof [7, to the integral equations for the Dirichlet problem to the $\mathcal{C}^{0}$ regularity of the integral equations for the Neumann problem. 
Proposition 6.1. Let $\partial \Omega \in \mathcal{C}^{k+1+\alpha}$ with $k \geq 0$ and $0<\alpha<1$. Let $0 \leq \beta \leq \alpha$.

(i) Let $p>1 / \alpha$. Then

$$
|\mathcal{K} \varphi|_{\alpha-1 / p} \leq C_{1+\alpha}|\varphi|_{L^{p}}, \quad\left|\mathcal{K}^{*} \varphi\right|_{\alpha^{\prime}} \leq C_{1+\alpha} C_{\alpha^{\prime}}|\varphi|_{L^{p}}
$$

for any $\alpha^{\prime} \leq \alpha-\frac{1}{p}$ with $\alpha^{\prime}<\alpha$.

(ii) Let $\mathcal{L}$ be one of $\mathcal{K},-\mathcal{K}, \mathcal{K}^{*}$, and $-\mathcal{K}^{*}$. Then

$$
\begin{gathered}
|\varphi|_{L^{p}} \leq C_{1+\alpha} C_{p}\left(|\varphi|_{L^{1}}+|\varphi+\mathcal{L} \varphi|_{L^{p}}\right), \quad p>1, \\
|\varphi|_{\beta} \leq C_{1+\alpha}\left(|\varphi|_{L^{1}}+|\varphi+\mathcal{L} \varphi|_{\beta}\right) .
\end{gathered}
$$

(iii) Let $\mathcal{L}=\mathcal{K}^{*}$ or $-\mathcal{K}^{*}$. Then $|\varphi|_{k+\beta} \leq C_{k+1+\alpha}\left(|\varphi|_{L^{1}}+|\varphi+\mathcal{L} \varphi|_{k+\beta}\right)$.

(iv) Let $\mathcal{L}=\mathcal{K}$ or $-\mathcal{K}$ and $1 \leq l \leq k+1$. Assume that $l \geq 2$ or $\partial_{\tau} \varphi \in L^{1}$. Then $|\varphi|_{l+\beta} \leq C_{k+1+\alpha}\left(|\varphi|_{L^{1}}+\left|\partial_{\tau} \varphi\right|_{L^{1}}+|\varphi+\mathcal{L} \varphi|_{l+\beta}\right)$.

Proof. (i) We adapt Kellogg's arguments in the proof of Proposition 5.5. Let $1 / p+$ $1 / q=1$. Decompose $\mathcal{K} \varphi\left(z_{2}\right)-\mathcal{K} \varphi\left(z_{1}\right)$ as

$$
\left\{\int_{\left|\zeta-z_{1}\right|<2\left|z_{2}-z_{1}\right|}+\int_{\left|\zeta-z_{1}\right|>2\left|z_{2}-z_{1}\right|}\right\} \varphi(\zeta)\left(K\left(z_{2}, \zeta\right)-K\left(z_{1}, \zeta\right)\right) d \sigma(\zeta)
$$

We estimate the first integral by $\left|K\left(z_{j}, \zeta\right)\right| \leq C\left|\zeta-z_{j}\right|^{\alpha-1}$ and get

$$
\left\{\int_{\left|\zeta-z_{1}\right|<2\left|z_{2}-z_{1}\right|}\left|K\left(z_{2}, \zeta\right)\right|^{q}+\left|K\left(z_{1}, \zeta\right)\right|^{q} d \sigma(\zeta)\right\}^{1 / q} \leq C\left|z_{2}-z_{1}\right|^{\alpha-1+\frac{1}{q}}
$$

We estimate the second integral by (4.2), i.e.,

$$
\left|K\left(z_{2}, \zeta\right)-K\left(z_{1}, \zeta\right)\right| \leq C\left|z_{2}-z_{1}\right|\left|\zeta-z_{1}\right|^{\alpha-2}
$$

for $\left|\zeta-z_{1}\right|>2\left|z_{2}-z_{1}\right|$. Thus

$$
\left\{\int_{\left|\zeta-z_{1}\right|>2\left|z_{2}-z_{1}\right|}\left|K\left(z_{2}, \zeta\right)-K\left(z_{1}, \zeta\right)\right|^{q} d \sigma(\zeta)\right\}^{1 / q} \leq C\left|z_{2}-z_{1}\right|^{\alpha-\frac{1}{p}}
$$

Therefore, $\varphi \in L^{p}$ implies $\mathcal{K} \varphi \in \mathcal{C}^{\alpha-\frac{1}{p}}$.

We now estimate $\mathcal{K}^{*} f$, for which we use Lemma 4.1, Thus, when $K$ is replaced by $K^{*}$ we still have (6.2) for $1 \leq p<\infty$ and (6.1). However, for $p=\infty$,

$$
\int_{\left|\zeta-z_{1}\right|>2\left|z_{2}-z_{1}\right|}\left|K\left(\zeta, z_{2}\right)-K\left(\zeta, z_{1}\right)\right| d \sigma(\zeta) \leq C\left|z_{2}-z_{1}\right|^{\alpha}\left(1+|\log | z_{2}-z_{1}||\right),
$$

which results in $\mathcal{K}^{*} \varphi \in \mathcal{C}^{\alpha^{\prime}}$.

(ii) We follow some standard estimates for compact integral operators ([3], p. 120; [10], p. 178). Let $L= \pm K$ or $\pm K^{*}$. Define $T_{L} \varphi=\int \varphi(\zeta) L(z, \zeta) d \sigma(\zeta)$. Let $\chi(z, \zeta)=1$ for $|z-\zeta|<\epsilon / 2$ and $\chi(z, \zeta)=0$ for $|z-\zeta|>\epsilon$. Let $p>1$ and $1 / p+1 / q=1$. We have

$$
\int_{\partial \Omega} \int_{\partial \Omega}|\varphi(\zeta)|^{p} \frac{d \sigma(\zeta) d \sigma(z)}{|z-\zeta|^{1-\alpha}} \leq C_{0} \alpha^{-1}|\varphi|_{L^{p}}^{p}
$$

Thus, we obtain $\left|T_{\chi L} \varphi\right|_{L^{p}} \leq C_{0} \alpha^{-1} \epsilon^{\alpha\left(1-\frac{1}{p}\right)}|\varphi|_{L^{p}}$ from

$$
\left|\int_{\partial \Omega} \varphi(\zeta) \chi L(z, \zeta) d \sigma(\zeta)\right| \leq\left(\int_{\partial \Omega}|\varphi(\zeta)|^{p} \frac{d \sigma(\zeta)}{|\zeta-z|^{1-\alpha}}\right)^{1 / p}\left(\int_{|z-\zeta|<\epsilon} \frac{d \sigma(\zeta)}{|\zeta-z|^{1-\alpha}}\right)^{1 / q} .
$$


Therefore, $I+T_{\chi L}: L^{p} \rightarrow L^{p}$ has an inverse with norm $<2$ when $C_{0} \alpha^{-1} \epsilon^{\alpha\left(1-\frac{1}{p}\right)}<$ $1 / 2$. Since $(1-\chi) L$ is continuous, it is easy to obtain

$$
\left|T_{(1-\chi) L} \varphi\right|_{L^{\infty}} \leq C_{\epsilon}|\varphi|_{L^{1}} .
$$

Using $\varphi=\left(I+T_{\chi L}\right)^{-1}\left(I+T_{L}\right) \varphi-\left(I+T_{\chi L}\right)^{-1} T_{(1-\chi) L} \varphi$, we estimate two inverses and obtain

$$
|\varphi|_{L^{p}} \leq C|\varphi+\mathcal{L} \varphi|_{L^{p}}+C_{\epsilon}|\varphi|_{L^{1}}
$$

When $\beta=0$, we get $\varphi \in L^{\infty}$ and hence $\varphi \in \mathcal{C}^{0}$ by (i). Assume that $\beta>0$. Using $\varphi=(\varphi+\mathcal{L} \varphi)-\mathcal{L} \varphi$, we obtain $|\varphi|_{\beta / 2} \leq C|\varphi+\mathcal{L} \varphi|_{\beta / 2}+C^{\prime}|\varphi|_{L^{1}}$, from which we get $|\varphi|_{\beta} \leq C_{1}|\varphi+\mathcal{L} \varphi|_{\beta}+C_{2}|\varphi|_{\beta / 2} \leq C_{3}|\varphi+\mathcal{L} \varphi|_{\beta}+C_{4}|\varphi|_{L^{1}}$

(iii) This follows from (5.44) with $j=0$ and (ii).

(iv) When $k \geq l \geq 2$, we know that $K$ is of class $\mathcal{C}^{1}$. Hence $\varphi \in \mathcal{C}^{1}$ if $\varphi+\mathcal{K} \varphi$ is additionally of class $\mathcal{C}^{1}$. Since $\partial_{\tau} \varphi \in L^{1}$, by Lemma 4.2 (ii) we get $\partial_{\tau} \varphi(z) \mp \mathcal{K}^{*} \partial_{\tau} \varphi=$ $\partial_{\tau}(\varphi \pm \mathcal{K} \varphi)$. The rest follows from (ii)-(iii).

For applications to integral equations with parameter (Lemma 7.1), we emphasize that the constants $C_{k+1+\alpha}$ in Proposition 6.1 depend only on $\left|\left(\hat{\gamma}^{\prime}\right)^{-1}\right|_{0}$ and $|\hat{\gamma}|_{k+1+\alpha}$ if $\partial \Omega$ is parameterized by $\hat{\gamma}$.

Proposition 6.2. Let $\partial \Omega \in \mathcal{C}^{1+\alpha}$. Let $e_{0}=1$ on $\partial \Omega$. For $i>0$, let $e_{i}=1$ on $\gamma_{i}$ and $e_{i}=0$ on $\partial \Omega \backslash \gamma_{i}$. Let $0 \leq \beta \leq \alpha$.

(i) Let $\mathcal{L}$ be one of $\mathcal{K},-\mathcal{K}, \mathcal{K}^{*}$, and $-\mathcal{K}^{*}$. Then $\varphi+\mathcal{L} \varphi=\psi \in \mathcal{C}^{\beta}(\partial \Omega)$ admits an $L^{1}$ solution $\varphi$ if and only if $\psi \perp \operatorname{ker}\left(I+\mathcal{L}^{*}\right)$. All $L^{1}$ solutions $\varphi$ are in $\mathcal{C}^{\beta}(\partial \Omega)$

(ii) $\left\{e_{1}, \ldots, e_{m}\right\}$ spans $\operatorname{ker}(I+\mathcal{K})$ and $e_{0}$ spans $\operatorname{ker}(I-\mathcal{K})$.

(iii) $\operatorname{ker}\left(I+\mathcal{K}^{*}\right) \cap \operatorname{ker}(I+\mathcal{K})^{\perp}=\{0\}$ and $\operatorname{ker}\left(I-\mathcal{K}^{*}\right) \cap \operatorname{ker}(I-\mathcal{K})^{\perp}=\{0\}$.

(iv) $\operatorname{ker}\left(I+\mathcal{K}^{*}\right)$ is spanned by $\left\{\phi_{1}, \ldots, \phi_{m}\right\}$, where $\phi_{i}$ satisfy $\int_{\gamma_{i}} \phi_{j} d \sigma=\delta_{i j}$ for $i, j>0$ and $\int_{\partial \Omega} \phi_{i} d \sigma=0$ for $i>0$. Moreover, $W \phi_{1}, \ldots, W \phi_{m}$ are locally constant on $\partial \Omega$ and vanish on the outer boundary of $\partial \Omega$, and $\left(\left.W \phi_{i}\right|_{\gamma_{j}}\right)_{1 \leq i, j \leq m}$ is non-singular when $m>0$.

(v) $\operatorname{ker}\left(I-\mathcal{K}^{*}\right)$ is spanned by $\phi_{0}$ and $W \phi_{0}$ is constant on $\partial \Omega$. Moreover, $\phi_{0}$ vanishes on $\partial \Omega \backslash \gamma_{0}, \int_{\partial \Omega} \phi_{0} d \sigma=1$, and $\phi_{0}$ depends only on $\gamma_{0}$.

Proof. (i)-(iii) The first assertion follows from the compactness of $\mathcal{L}$ on $L^{2}$ (10], p. 162, p. 167) and $\varphi \in \mathcal{C}^{\beta}$ follows from Proposition 6.1 (ii). The proof of (ii) is in 3 (p. 135). For (iii), assume that $\psi \in \operatorname{ker}\left(I+\mathcal{K}^{*}\right) \cap \operatorname{ker}(I+\mathcal{K})$. We have $\psi+\mathcal{K}^{*} \psi=0$, and by (i) $\psi=\varphi+\mathcal{K}^{*} \varphi$. For $\psi \in \operatorname{ker}\left(I-\mathcal{K}^{*}\right) \cap \operatorname{ker}(I-\mathcal{K})$, we have $\psi-\mathcal{K}^{*} \psi=0$ and $\psi=\varphi-\mathcal{K}^{*} \varphi$. In both cases, we have $\varphi, \psi \in \mathcal{C}^{\alpha}$. Then $W \varphi$ and $W \psi$ are in $\mathcal{C}^{1+\alpha}$ by Lemma 3.2 (iii). One can show that $\psi=0$; see [3] (p. 137), where the use of Green's identities merely requires that $\partial \Omega, W \varphi, W \psi$ be of class $\mathcal{C}^{1+\alpha}$.

(iv) By compactness of $\mathcal{L}$, we have $\operatorname{dim} \operatorname{ker}\left(I+\mathcal{L}^{*}\right)=\operatorname{dim} \operatorname{ker}(I+\mathcal{L})=1([3]$, p. 24). Note that if $\phi_{1}, \ldots, \phi_{m}$ span $\operatorname{ker}\left(I+\mathcal{K}^{*}\right)$, the matrix $A=\left(\int_{\gamma_{i}} \phi_{j} d \sigma\right)_{1 \leq i, j \leq m}$ must have rank $m$. Indeed if $\varphi=c_{1} \phi_{1}+\cdots+c_{m} \phi_{m}$ is orthogonal to $e_{1}, \ldots, e_{m}$, then by (i) and (iii), $\varphi=0$. With $A$ being non-singular, we can normalize $\phi_{i}$ such that $A$ is the identity matrix. This verifies the first assertion. To show that $W \phi_{i}$ are locally constant on $\partial \Omega$, we integrate $\phi_{i}+\mathcal{K}^{*} \phi_{i}=0$ and get $\int_{\partial \Omega} \phi_{i} d \sigma=0$ for $i>0$. This shows that $\phi_{i} \in \operatorname{ker}\left(I+\mathcal{K}^{*}\right)$. Hence, $W \phi_{i}$ is locally constant on $\Omega^{\prime}$ and vanishes on the unbounded component of $\Omega^{\prime}$. By the continuity of $W \phi_{i}$, it is constant on the inner boundary of $\partial \Omega$ and vanishes on the outer boundary. Assume for the sake 
of contradiction that $\left(\left.W \phi_{i}\right|_{\gamma_{j}}\right)_{1 \leq i, j \leq m}$ is singular. Since $W \phi_{i}$ are constants on $\gamma_{i}$ and vanish on $\gamma_{0}$, then $W \phi_{1}, \ldots, W \phi_{m}$ are linearly dependent on $\partial \Omega$. Therefore, for some $c_{i}$ which are not all zero, we have $W\left(c_{1} \phi_{1}+\cdots+c_{m} \phi_{m}\right)=0$ on $\partial \Omega$. This implies that $c_{1} \phi_{1}+\cdots+c_{m} \phi_{m}$ is in $\operatorname{ker}\left(I+\mathcal{K}^{*}\right) \cap \operatorname{ker}\left(I-\mathcal{K}^{*}\right)$. Since $\phi_{1}, \ldots, \phi_{m}$ form a basis, we get $c_{i}=0$ for all $i$, a contradiction.

(v) By (iii), we know that if $\phi_{0}$ spans $\operatorname{ker}\left(I-\mathcal{K}^{*}\right)$, then $\int_{\partial \Omega} \phi_{0} d \sigma \neq 0$. Let $\phi_{0}$ be the unique element in $\operatorname{ker}\left(I-\mathcal{K}^{*}\right)$ such that $\int_{\partial \Omega} \phi_{0} d \sigma=1$. We want to show that $\phi_{0}=0$ on $\gamma_{j}$ for $j>0$ and that $\phi_{0}$ depends only on $\gamma_{0}$.

Let $\Omega_{0}$ be the bounded domain bounded by the outer boundary $\gamma_{0}$ of $\Omega$. Let $\phi$, with $\int_{\gamma_{0}} \phi d \sigma=1, \operatorname{span} \operatorname{ker}\left(I-\mathcal{K}_{0}^{*}\right) \subset L^{2}\left(\partial \Omega_{0}\right)$. Here $\mathcal{K}_{0}^{*}(\zeta, z)=\frac{1}{\pi} \partial_{\tau_{\zeta}} \arg (\zeta-z)$ for $\zeta, z \in \partial \Omega_{0}$. Let $\hat{W} \phi$ be the single-layer distribution with density $\phi$ on $\gamma_{0}$. Since $\hat{W} \phi$ is constant on $\Omega_{0}$, then $\partial_{\nu} \hat{W} \phi=0$ for the normal vector $\nu$ of any $\mathcal{C}^{1}$ curve $\gamma$ in $\Omega_{0}$. This shows that $\operatorname{ker}\left(I-\mathcal{K}^{*}\right)$ is spanned by $\tilde{\phi}$ if $\tilde{\phi}$ equals $\phi$ on $\gamma_{0}$ and is zero on $\partial \Omega \backslash \gamma_{0}$. The condition $\int_{\partial \Omega} \phi_{0} d \sigma=1$ implies that $\phi_{0}=\tilde{\phi}$.

For convenience, we will use $\left\{e_{1}, \ldots, e_{m}\right\},\left\{e_{0}\right\},\left\{\phi_{1}, \ldots, \phi_{m}\right\}$, and $\left\{\phi_{0}\right\}$ for bases of $\operatorname{ker}(I+\mathcal{K}), \operatorname{ker}(I-\mathcal{K}), \operatorname{ker}\left(I+\mathcal{K}^{*}\right)$, and $\operatorname{ker}\left(I-\mathcal{K}^{*}\right)$, respectively.

Lemma 6.3. Let $\partial \Omega \in \mathcal{C}^{1+\alpha}$ and $0 \leq \beta \leq \alpha$. Let $\mathcal{L}=\mathcal{K}$ or $-\mathcal{K}$. If $\varphi+\mathcal{L} \varphi=g \in$ $\mathcal{C}^{1+\beta}(\partial \Omega)$ and $\varphi \perp \operatorname{ker}(I+\mathcal{L})$, then $\varphi \in \mathcal{C}^{1+\beta}(\partial \Omega)$ and it is determined by

$$
\begin{gathered}
\varphi=\hat{\varphi}+c_{0} e_{0}+c_{1} e_{1}+\cdots+c_{m} e_{m}, \\
\varphi_{1}-\mathcal{L}^{*} \varphi_{1}=\partial_{\tau} g, \quad \varphi_{1} \in \operatorname{ker}\left(I-\mathcal{L}^{*}\right)^{\perp} \cap \operatorname{ker}(I+\mathcal{L})^{\perp}, \\
\partial_{\tau} \hat{\varphi}=\varphi_{1}-d_{0} \phi_{0}-d_{1} \phi_{1}-\cdots-d_{m} \phi_{m}, \quad \int_{\gamma_{i}} \hat{\varphi} d \sigma=0, \quad i \geq 0 .
\end{gathered}
$$

Moreover, $c_{i}$ and $d_{i}$ are determined as follows:

(i) If $\mathcal{L}=\mathcal{K}$, then

$$
\begin{gathered}
c_{0}=\frac{1}{2 l^{2}} \int_{\partial \Omega}(g-\mathcal{L} \hat{\varphi}) d \sigma, \quad c_{1}=\cdots=c_{m}=-c_{0}, \\
d_{0}=\int_{\partial \Omega} \varphi_{1} e_{0} d \sigma, \quad d_{1}=\cdots=d_{m}=0 .
\end{gathered}
$$

(ii) If $\mathcal{L}=-\mathcal{K}$, then

$$
\begin{gathered}
c_{i}=\frac{1}{2 l_{i}^{2}} \int_{\gamma_{i}}(g-\mathcal{L} \hat{\varphi}) d \sigma, \quad i \geq 1, \quad c_{0}=-\frac{1}{2 l^{2}} \int_{\partial \Omega \backslash \gamma_{0}}(g-\mathcal{L} \hat{\varphi}) d \sigma, \\
d_{i}=\int_{\partial \Omega} \varphi_{1} e_{i} d \sigma, \quad i \geq 1, \quad d_{0}=0 .
\end{gathered}
$$

Proof. (i) Assume that $\varphi+\mathcal{K} \varphi=g \in \mathcal{C}^{1+\beta}$. Recall that $e_{1}, \ldots, e_{m} \operatorname{span} \operatorname{ker}(I+\mathcal{K})$. Since $\partial_{\tau} g \perp \operatorname{ker}(I-\mathcal{K})$, there exists $\varphi_{1} \in \mathcal{C}^{\beta} \cap \operatorname{ker}\left(I-\mathcal{K}^{*}\right)^{\perp}$ such that $\varphi_{1}-\mathcal{K}^{*} \varphi_{1}=$ $\partial_{\tau} g$. Let $j>0$. Since $\int_{\gamma_{j}} \mathcal{K}^{*} \varphi_{1} d \sigma=-\int_{\gamma_{j}} \varphi_{1} d \sigma$, then $\int_{\gamma_{j}} \varphi_{1} d \sigma=0$. Recall that $\phi_{0}=0$ on $\gamma_{j}$ and $\int_{\gamma_{0}} \phi_{0} d \sigma=1$. Let $d_{0}=\int_{\partial \Omega} \varphi_{1} d \sigma$. Then $\tilde{\varphi}_{1}=\varphi_{1}-d_{0} \phi_{0}$ is orthogonal to $e_{0}, \ldots, e_{m}$, and hence there is a unique $\hat{\varphi} \in \mathcal{C}^{1+\beta}$ such that $\tilde{\varphi}_{1}=\partial_{\tau} \hat{\varphi}$ and $\int_{\gamma} \hat{\varphi} e_{i} d \sigma=0$ for all $i \geq 0$. Thus, we obtain

$$
\begin{aligned}
\partial_{\tau}(\varphi+K \varphi) & =\partial_{\tau} g=\tilde{\varphi}_{1}-\mathcal{K}^{*} \tilde{\varphi}_{1}=\partial_{\tau} \hat{\varphi}+d_{0} \phi_{0}-\mathcal{K}^{*}\left(\partial_{\tau} \hat{\varphi}+d_{0} \phi_{0}\right) \\
& =\partial_{\tau}(\hat{\varphi}+K \hat{\varphi}) .
\end{aligned}
$$


Hence, $\varphi-\hat{\varphi}+\mathcal{K}(\varphi-\hat{\varphi})=2 c_{0}+\sum_{i>0} \tilde{c}_{i} e_{i}$. We rewrite it as

$$
\left(\varphi-\hat{\varphi}-c_{0}\right)+\mathcal{K}\left(\varphi-\hat{\varphi}-c_{0}\right)=\sum_{i>0} \tilde{c}_{i} e_{i}
$$

Being in the range of $I+\mathcal{K}$, the right-hand side must be orthogonal to $\operatorname{ker}\left(I+\mathcal{K}^{*}\right)$. Hence, $\tilde{c}_{i}=0$ and consequently $\varphi-\hat{\varphi}-c_{0}=\sum_{j>0} c_{j} e_{j}$. This shows that $\varphi \in \mathcal{C}^{1+\beta}$. Since $\varphi$ and $\hat{\varphi}$ are orthogonal to $e_{i}$ for $i>0$, then $c_{i}+c_{0}=0$. We substitute $\hat{\varphi}+c_{0} e_{0}+\cdots+c_{m} e_{m}$ for $\varphi$ in $\varphi+\mathcal{K} \varphi=g$ to get $g=\hat{\varphi}+\mathcal{K} \hat{\varphi}+2 c_{0} e_{0}$. Therefore, $2 c_{0} l^{2}=\int_{\partial \Omega}(g-\mathcal{K} \hat{\varphi}) d \sigma$.

(ii) Assume that $\varphi-\mathcal{K} \varphi=g \in \mathcal{C}^{1+\beta}$. We find $\varphi_{1} \in \mathcal{C}^{\beta} \cap \operatorname{ker}\left(I+\mathcal{K}^{*}\right)^{\perp}$ such that $\varphi_{1}+\mathcal{K}^{*} \varphi_{1}=\partial_{\tau} g$. By $\int_{\partial \Omega} \mathcal{K}^{*} \varphi_{1} d \sigma=\int_{\partial \Omega} \varphi_{1} d \sigma$, we get $\int_{\partial \Omega} \varphi_{1} d \sigma=0$. Since $\phi_{j} \in \operatorname{ker}\left(I+\mathcal{K}^{*}\right)$ satisfy $\int_{\gamma_{i}} \phi_{j} d \sigma=\delta_{i j}$ for $i, j>0$, then for $d_{j}=\int_{\gamma_{j}} \varphi_{1} d \sigma, \tilde{\varphi}_{1}=$ $\varphi_{1}-d_{1} \phi_{1}-\cdots-d_{m} \phi_{m}$ is orthogonal to $e_{1}, \ldots, e_{m}$. We still have $\tilde{\varphi}_{1}+\mathcal{K}^{*} \tilde{\varphi}_{1}=\partial_{\tau} g$; in particular, $\int_{\gamma_{0}} \tilde{\varphi}_{1} d \sigma=\int_{\partial \Omega} \tilde{\varphi}_{1} d \sigma=0$. We write $\tilde{\varphi}_{1}=\partial_{\tau} \hat{\varphi}$ with $\int_{\gamma_{j}} \hat{\varphi} d \sigma=0$ for $j \geq 0$. As in (ii), we get $\partial_{\tau}(\varphi-\hat{\varphi}-\mathcal{K}(\varphi-\hat{\varphi}))=0$, and hence

$$
(\varphi-\hat{\varphi})-\mathcal{K}(\varphi-\hat{\varphi})=\tilde{c}_{0}+2 \sum_{i=1}^{m} c_{i} e_{i}
$$

The right-hand side must be orthogonal to $\operatorname{ker}\left(I-\mathcal{K}^{*}\right)$, the span of $\phi_{0}$. As $\phi_{0}$ vanishes on $\gamma_{1} \cup \cdots \cup \gamma_{m}$ by Proposition $6.2(\mathrm{v})$, we obtain $\tilde{c}_{0}=\tilde{c}_{0} \int_{\partial \Omega} \phi_{0} d \sigma=0$. Then $\varphi-\hat{\varphi}-\sum_{j>0} c_{j} e_{j} \in \operatorname{ker}(I-\mathcal{K})$, so it is a constant $c_{0}$. Therefore, $\varphi \in \mathcal{C}^{1+\beta}$. Also, $g=\varphi-\mathcal{K} \varphi=\hat{\varphi}-\mathcal{K} \hat{\varphi}+2\left(c_{1} e_{1}+\cdots+c_{m} e_{m}\right)$. We get $2 c_{i} l_{i}^{2}=\int_{\gamma_{i}}(g-\mathcal{K} \hat{\varphi}) d \sigma$ for $i>0$. Using $0=\left\langle\varphi, e_{0}\right\rangle=\sum_{i>0} c_{i}\left|l_{i}\right|^{2}+c_{0} l^{2}$, we get the formula for $c_{0}$.

The above lemma allows us to study integral equations for the planar Dirichlet problem via integral equations for the Neumann problem. We now strengthen Proposition 6.1 (iv) as follows.

Corollary 6.4. Let $l \geq 1,0<\alpha<1$ and $0 \leq \beta \leq \alpha$. Let $\Omega$ be a bounded domain with $\partial \Omega \in \mathcal{C}^{l+\alpha}$. Let $\mathcal{L}$ be $\mathcal{K}$ or $-\mathcal{K}$. If $\varphi+\overline{\mathcal{L}} \varphi \in \overline{\mathcal{C}}^{l+\beta}(\partial \Omega)$, then $\varphi \in \mathcal{C}^{l+\beta}(\partial \Omega)$.

\section{REgularity of SOLUTIONS FOR INTEGRAL EQUATIONS WITH PARAMETER}

We first describe the main difficulty if we use the Fredholm resolvent directly to formulate our solutions for domains depending on a parameter. Such a formulation via the resolvent for the Dirichlet problem on a fixed domain is in [8]. Assume for simplicity that $\partial \Omega$ is $\mathcal{C}^{2}$, and parameterize $\partial \Omega$ by $\gamma(t)$ in arc-length. The kernel $K(s, t)=\frac{1}{\pi} \partial_{t} \arg (\gamma(s)-\gamma(t))$ is then continuous, and the resolvent $L(s, t, z)$ satisfies

$$
K(s, t)=L(s, t, z)+z \int_{0}^{l} L(s, r, z) K(r, t) d r .
$$

It is a basic theorem of Fredholm that there exists $\delta(z)$ with $\delta(0)=1$ such that $\delta(z)$ and $\delta(z) L(s, t, z)$ are entire functions in $z$ (see, e.g., [7]). It is known that $L(s, t, z)$ is analytic at $z=1$ when $\Omega$ is simply connected (see [7, or [3], p. 133); by a theorem of Plemelj [12, it has a simple pole at $z=1$ otherwise. One can verify that $\delta(1) \neq 0$ when $\Omega$ is simply connected $(9$, p. 294), and in this case the zeros of $\delta$ do not accumulate at 1 as domains vary. However, we do not know if the zeros of $\delta$ accumulate at 1 as $\delta$ varies with $\Omega$, when $\Omega$ is multi-connected. Without resolving this issue, we will estimate solutions by taking the limit and differentiating in $\lambda$ on the integral equations directly. 
This section consists of three results. Lemma 7.1] shows the uniform boundedness of solutions of integral equations in $L^{p}$ and Hölder norms; Lemma 7.3 provides formulae to differentiate the integral equations; Proposition 7.4 contains the estimates for the solutions of the integral equations.

Recall that for a family of functions $f^{\lambda}$ on $\partial \Omega \in \mathcal{C}^{k+\alpha} \cap \mathcal{C}^{1}$, we define for $k \geq j$

$$
\|f\|_{k+\alpha, j}=\max _{i \leq j, \lambda}\left|\partial_{\lambda}^{i} f^{\lambda}\right|_{k-i+\alpha}, \quad\left\|f^{\mu}-f^{\lambda}\right\|_{k+\alpha, j}=\max _{i \leq j}\left|\partial_{\mu}^{i} f^{\mu}-\partial_{\lambda}^{i} f^{\lambda}\right|_{k-i+\alpha} .
$$

For a family of embeddings $z \rightarrow \gamma^{\lambda}(z)$ from $\partial \Omega$ onto $\partial \Omega^{\lambda}$, we use the notation $z^{\lambda}=\gamma^{\lambda}(z)$ and

$$
g(z, \lambda)=g^{\lambda}\left(\gamma^{\lambda}(z)\right)
$$

Let $\left\{\mathcal{L}^{\lambda}\right\}$ be one of $\left\{\mathcal{K}^{\lambda}\right\},\left\{-\mathcal{K}^{\lambda}\right\},\left\{\left(\mathcal{K}^{\lambda}\right)^{*}\right\},\left\{-\left(\mathcal{K}^{\lambda}\right)^{*}\right\}$, and let $\left\{\ell_{1}^{\lambda}, \ldots, \ell_{n}^{\lambda}\right\}$ be the canonical basis of $\operatorname{ker}\left(I+\mathcal{L}^{\lambda}\right)$, described after the proof of Proposition 6.2. Define

$$
\left(\left\langle\varphi, \ell_{i}\right\rangle\right)^{\lambda}=\left\langle\varphi^{\lambda}, \ell_{i}^{\lambda}\right\rangle=\int_{\partial \Omega^{\lambda}} \varphi^{\lambda} \ell_{i}^{\lambda} d \sigma^{\lambda} .
$$

Lemma 7.1. Let $\gamma^{\lambda}$ embed $\partial \Omega$ onto $\partial \Omega^{\lambda}$ with $\gamma \in \mathcal{B}^{1+\alpha, 0}(\partial \Omega)$. Let $0<\alpha<1$ and $0 \leq \beta \leq \alpha$. Let $\varphi^{\lambda} \in L^{1}\left(\partial \Omega^{\lambda}\right)$ and define $\psi_{i}^{\lambda}$ according to the following two cases:

a) Let $\left\{\mathcal{L}^{\lambda}\right\}$ be $\left\{\mathcal{K}^{\lambda}\right\}$ or $\left\{-\mathcal{K}^{\lambda}\right\}$. Also, let

$$
\varphi^{\lambda}+\left(\mathcal{L}^{\lambda}\right)^{*} \varphi^{\lambda}=\psi_{0}^{\lambda}, \quad\left\langle\varphi^{\lambda}, \ell_{i}^{\lambda}\right\rangle=\psi_{i}^{\lambda}, \quad 1 \leq i \leq n .
$$

b) Let $\left\{\mathcal{L}^{\lambda}\right\}$ be one of $\left\{\mathcal{K}^{\lambda}\right\},\left\{-\mathcal{K}^{\lambda}\right\},\left\{\left(\mathcal{K}^{\lambda}\right)^{*}\right\}$, and $\left\{-\left(\mathcal{K}^{\lambda}\right)^{*}\right\}$. Also, let

$$
\varphi^{\lambda}+\mathcal{L}^{\lambda} \varphi^{\lambda}=\psi_{0}^{\lambda}, \quad\left\langle\varphi^{\lambda}, \ell_{i}^{\lambda}\right\rangle=\psi_{i}^{\lambda}, \quad 1 \leq i \leq n .
$$

Then the following hold:

(i) Let $1 / \alpha<p \leq \infty$. Suppose that $\lambda \rightarrow \psi_{0}^{\lambda} \circ \gamma^{\lambda} \in L^{p}(\partial \Omega)$ and $\lambda \rightarrow \psi_{i}^{\lambda}$ are bounded (resp. continuous) maps on $[0,1]$. Then $\lambda \rightarrow \varphi^{\lambda} \circ \gamma^{\lambda} \in L^{p}(\partial \Omega)$ is bounded (resp. continuous) on $[0,1]$.

(ii) If $\psi_{0}^{\mu} \in L^{p}\left(\partial \Omega^{\mu}\right)$ and $\psi_{0}^{\lambda} \in L^{p}\left(\partial \Omega^{\lambda}\right)$ with $1<p \leq \infty$, then

$$
\begin{aligned}
\mid \varphi(\cdot, \mu) & -\left.\varphi(\cdot, \lambda)\right|_{L^{p}} \leq C_{1+\alpha, 0} C_{p}\left(|\varphi(\cdot, \mu)-\varphi(\cdot, \lambda)|_{L^{1}}+\left|\psi_{0}(\cdot, \mu)-\psi_{0}(\cdot, \lambda)\right|_{L^{p}}\right. \\
& \left.+\left(\left|\left(\psi_{0}(\cdot, \mu), \psi_{0}(\cdot, \lambda)\right)\right|_{L^{p}}+|(\varphi(\cdot, \mu), \varphi(\cdot, \lambda))|_{L^{1}}\right)\left|\gamma^{\mu}-\gamma^{\lambda}\right|_{1+\alpha}\right) .
\end{aligned}
$$

(iii) If $\psi_{0}^{\mu} \in \mathcal{C}^{\beta}\left(\partial \Omega^{\mu}\right)$ and $\psi_{0}^{\lambda} \in \mathcal{C}^{\beta}\left(\partial \Omega^{\lambda}\right)$, then

$$
\begin{aligned}
|\varphi(\cdot, \mu)-\varphi(\cdot, \lambda)|_{\beta} \leq & C_{1+\alpha, 0}\left(|\varphi(\cdot, \mu)-\varphi(\cdot, \lambda)|_{L^{1}}+\left|\psi_{0}(\cdot, \mu)-\psi_{0}(\cdot, \lambda)\right|_{\beta}\right. \\
& \left.+\left(\left|\psi_{0}^{\lambda}\right|_{\beta}+\left|\varphi^{\lambda}\right|_{L^{1}}\right)\left|\gamma^{\mu}-\gamma^{\lambda}\right|_{1+\alpha}\right) .
\end{aligned}
$$

Proof. We first verify the assertions for case a). The verification for b) will be simpler, after we establish $\phi_{i} \in \mathcal{B}^{\alpha, 0}\left(\partial \Omega_{\gamma}\right)$ via (i) of case a). The proof of (i) is given in Steps 1 and 2 below. The proofs of (ii) and (iii) are in Step 3.

Step 1 (Boundedness in $L^{p}$ norms). Fix $1 / \alpha<p \leq \infty$. We are given

$$
\begin{gathered}
\varphi(\zeta, \lambda)+\int_{\eta \in \partial \Omega} \varphi(\eta, \lambda) L^{\lambda}\left(\eta^{\lambda}, \zeta^{\lambda}\right) d \sigma^{\lambda}\left(\eta^{\lambda}\right)=\psi_{0}(\zeta, \lambda), \\
\int_{\eta \in \partial \Omega} \varphi(\eta, \lambda) \ell_{i}^{\lambda} d \sigma^{\lambda}\left(\eta^{\lambda}\right)=\psi_{i}^{\lambda}, \quad 1 \leq i \leq n .
\end{gathered}
$$


Assume for the sake of contradiction that $A_{j}=\left|\varphi^{\lambda_{j}}\right|_{L^{p}} \rightarrow \infty$ for some $\lambda_{j} \rightarrow 0$. Normalize in the $L^{p}$ norm by letting $\tilde{\varphi}^{\lambda_{j}}=A_{j}^{-1} \varphi^{\lambda_{j}}$ and $\tilde{\psi}_{i}^{\lambda_{j}}=A_{j}^{-1} \psi_{i}^{\lambda_{j}}$. We get

$$
\begin{gathered}
\tilde{\varphi}\left(\zeta, \lambda_{j}\right)+\int_{\partial \Omega} \tilde{\varphi}\left(\eta, \lambda_{j}\right) L^{\lambda_{j}}\left(\eta^{\lambda_{j}}, \zeta^{\lambda_{j}}\right) d \sigma^{\lambda_{j}}\left(\eta^{\lambda_{j}}\right)=\tilde{\psi}_{0}\left(\zeta, \lambda_{j}\right), \\
\left|\tilde{\varphi}\left(\cdot, \lambda_{j}\right)\right|_{L^{p}}=1, \quad \int_{\partial \Omega} \tilde{\varphi}\left(\eta, \lambda_{j}\right) \ell_{i}^{\lambda_{j}} d \sigma^{\lambda_{j}}\left(\eta^{\lambda_{j}}\right)=\tilde{\psi}_{i}^{\lambda_{j}}
\end{gathered}
$$

Since the $L^{p}$ norms of $\tilde{\varphi}^{\lambda_{j}}$ are bounded, by Proposition 6.1 (i) the $\mathcal{C}^{\alpha / 2}$-norms of $\left(\mathcal{L}^{\lambda_{j}}\right)^{*} \varphi^{\lambda_{j}}$ on $\partial \Omega^{\gamma_{j}}$ are bounded too. Thus, $\left(\left(\mathcal{L}^{\lambda_{j}}\right)^{*} \varphi^{\lambda_{j}}\right) \circ \gamma^{\lambda_{j}}$ have bounded $\mathcal{C}^{\alpha / 2_{-}}$ norms on $\partial \Omega$. Passing to a subsequence if necessary, $\left(\left(\mathcal{L}^{\lambda_{j}}\right)^{*} \varphi^{\lambda_{j}}\right) \circ \gamma^{\lambda_{j}}$ converges uniformly on $\partial \Omega$. Since $\tilde{\psi}_{0}\left(\cdot, \lambda_{j}\right)$ converges to 0 in the $L^{p}$ norm, (7.4) implies that $\tilde{\varphi}\left(\cdot, \lambda_{j}\right)$ converges to some $\varphi_{*}=\varphi^{*} \circ \gamma^{0}$ in the $L^{p}$ norm. Recall that $d \sigma^{\lambda}\left(z^{\lambda}\right)=$ $a^{\lambda}(z) d \sigma(z)$ with $a^{\lambda}(z)=\left|\partial_{\tau} \gamma^{\lambda}(z)\right|$. Since $a^{\lambda_{j}}$ converges to $a^{0}$ in sup norm, then $\tilde{\varphi}\left(\cdot, \lambda_{j}\right) a^{\lambda_{j}}(\cdot)$ approaches to $\varphi_{*} a^{0}(\cdot)$ in the $L^{p}$ norm. Decompose

$$
\begin{aligned}
& \left|\int_{\partial \Omega} \tilde{\varphi}\left(\eta, \lambda_{j}\right) L^{\lambda_{j}}\left(\eta^{\lambda_{j}}, \zeta^{\lambda_{j}}\right) d \sigma^{\lambda_{j}}\left(\eta^{\lambda_{j}}\right)-\int_{\partial \Omega} \varphi_{*}(\eta) L^{0}\left(\eta^{0}, \zeta^{0}\right) d \sigma^{0}\left(\eta^{0}\right)\right| \\
& \quad \leq\left|\int_{\partial \Omega}\left(\tilde{\varphi}\left(\eta, \lambda_{j}\right) a^{\lambda_{j}}(\eta)-\varphi_{*}(\eta) a^{0}(\eta)\right) L^{\lambda_{j}}\left(\eta^{\lambda_{j}}, \zeta^{\lambda_{j}}\right) d \sigma(\eta)\right| \\
& \quad+\left|\int_{\partial \Omega} \varphi_{*}(\eta) a^{0}(\eta)\left(L^{\lambda_{j}}\left(\eta^{\lambda_{j}}, \zeta^{\lambda_{j}}\right)-L^{0}\left(\eta^{0}, \zeta^{0}\right)\right) d \sigma(\eta)\right|=I_{j}^{\prime}(z)+I_{j}^{\prime \prime}(z) .
\end{aligned}
$$

From $p>1 / \alpha,\left|K^{\lambda}\left(\zeta^{\lambda}, z^{\lambda}\right)\right| \leq C|\zeta-z|^{\alpha-1}$, and Hölder inequalities, we see that $I_{j} \rightarrow 0$ in $L^{p}$ as $\lambda_{j} \rightarrow 0$. From Hölder inequalities and the dominated convergence theorem, we see that $I_{j}^{\prime \prime} \rightarrow 0$ in $L^{p}$ for $\lambda_{j} \rightarrow 0$ also. Thus, letting $j$ tend to $\infty$ in (7.4)-(7.5), we get

$$
\begin{gathered}
\varphi^{*}\left(\zeta^{0}\right)+\int_{\partial \Omega} \varphi^{*}\left(\eta^{0}\right) L^{0}\left(\eta^{0}, \zeta^{0}\right) d \sigma^{0}\left(\eta^{0}\right)=0 \\
\left|\varphi^{*}\right|_{L^{p}}=1, \quad \int_{\partial \Omega} \varphi^{*}\left(\eta^{0}\right) \ell_{i}^{0} d \sigma^{0}\left(\eta^{0}\right)=0, \quad i=1, \ldots, n .
\end{gathered}
$$

By Proposition 6.2 (iii), the first and last $n$ identities imply that $\varphi^{*}=0$. The latter contradicts to the second identity. Therefore $\left\{\left|\varphi^{\lambda}\right|_{L^{p}}\right\}$ is bounded. By Proposition 6.1 (i) and (ii), we obtain

$$
\begin{gathered}
\left|\left(\mathcal{L}^{\lambda}\right)^{*} \varphi^{\lambda}\right|_{\alpha / 2} \leq C_{1+\alpha}\left|\varphi^{\lambda}\right|_{0}, \\
\left|\varphi^{\lambda}\right|_{\beta} \leq C_{1+\alpha}\left(\left|\varphi^{\lambda}\right|_{L^{1}}+\left|\psi_{0}^{\lambda}\right|_{\beta}\right) .
\end{gathered}
$$

Step 2 (Continuity in $L^{p}$ norms). Fix $1 / \alpha<p \leq \infty$. Assume for the sake of contradiction that $\left|\varphi\left(\cdot, \lambda_{j}\right)-\varphi(\cdot, 0)\right|_{L^{p}} \geq \delta>0$ for a sequence $\lambda_{j}$ tending to zero. By (7.6), passing to a subsequence if necessary, we may assume that the sequence of continuous functions $\left(\left(\mathcal{L}^{\lambda_{j}}\right)^{*} \varphi^{\lambda_{j}}\right) \circ \gamma^{\lambda_{j}}$ converges uniformly as $\lambda_{j} \rightarrow 0$. Hence by (17.3), $\varphi\left(\cdot, \lambda_{j}\right)$ converges to $\varphi_{*}=\varphi^{*} \circ \gamma^{0}$ in $L^{p}$. We have $\left|\varphi(\cdot, 0)-\varphi^{*}(\cdot)\right|_{L^{p}} \geq \delta$. By the same arguments in Step 1 we know that $\varphi^{*}, \varphi^{0}$ satisfy the same equations,

$$
\begin{gathered}
\varphi^{0}\left(\zeta^{0}\right)+\int_{\partial \Omega} \varphi^{0}\left(\eta^{0}\right) L^{0}\left(\eta^{0}, \zeta^{0}\right) d \sigma^{0}\left(\eta^{0}\right)=\psi_{0}^{0}\left(\zeta^{0}\right), \\
\int_{\partial \Omega} \varphi^{0}\left(\eta^{0}\right) \ell_{i}^{0} d \sigma^{0}\left(\eta^{0}\right)=\psi_{i}^{0}, \quad 0 \leq i \leq n .
\end{gathered}
$$


By Proposition 6.2 (iii), $\varphi^{0}=\varphi^{*}$, a contradiction. This proves the continuity of $\varphi(z, \lambda)$ in the $L^{p}$ norm.

We proceed to repeat Steps 1 and 2 for case b). From case a), we know that $\phi_{0}, \ldots, \phi_{m}$ are of class $\mathcal{B}^{\alpha, 0}$. Thus the basis $\left\{\ell_{1}, \ldots, \ell_{n}\right\}$ of $\operatorname{ker}(I+\mathcal{L})$ is of class $\mathcal{B}^{\alpha, 0}$ in all cases.

We are given

$$
\varphi^{\lambda}+\mathcal{L}^{\lambda} \varphi^{\lambda}=\psi_{0}^{\lambda}, \quad \int_{\eta \in \partial \Omega} \varphi^{\lambda} \ell_{i}^{\lambda} d \sigma^{\lambda}\left(\eta^{\lambda}\right)=\psi_{i}^{\lambda}, \quad 1 \leq i \leq n
$$

We first repeat Step 1, which is simpler now. Assume for the sake of contradiction that there exists a sequence $\lambda_{j}$, approaching to 0 , such that $\left|\varphi_{j}^{\lambda_{j}}\right|_{L^{p}}=B_{j}$ tends to $\infty$. Then $\tilde{\varphi}_{j}=B_{j}^{-1} \varphi_{j}^{\lambda_{j}}$ has bounded $L^{p}$ norms and $\mathcal{L}^{\lambda_{j}} \tilde{\varphi}_{j}$ has bounded $\mathcal{C}^{\alpha / 2}$ norms. Passing to a subsequence if necessary, we may assume that $\left(\mathcal{L}^{\lambda_{j}} \tilde{\varphi}_{j}\right) \circ \gamma^{\lambda_{j}}$ converges uniformly on $\partial \Omega$. Hence $\tilde{\varphi}_{j} \circ \gamma^{\lambda_{j}}=\left(B_{j}^{-1} \psi_{j}-\mathcal{L}^{\lambda_{j}} \tilde{\varphi}_{j}\right) \circ \gamma^{\lambda_{j}}$ converges to $\varphi_{*}=\varphi^{*} \circ \gamma^{0}$ in $L^{p}(\partial \Omega)$. Reasoning as in Step 1 shows that $\varphi^{*}$ satisfies

$$
\varphi^{*}+\mathcal{L}^{0} \varphi^{*}=0, \quad \varphi^{*} \perp \operatorname{ker}\left(I+\mathcal{L}^{0}\right), \quad\left|\varphi^{*}\right|_{L^{p}}=1 .
$$

The first two expressions imply that $\varphi^{*}=0$, a contradiction. This shows that $\varphi^{\lambda}$ have bounded $L^{p}$ norms. Thus the $\mathcal{C}^{\alpha / 2}$ norms of $\mathcal{L}^{\lambda} \varphi^{\lambda}$ on $\partial \Omega^{\lambda}$ are bounded, and every sequence $\varphi^{\lambda_{j}} \circ \gamma^{\lambda_{j}}(z)$ with $\lambda_{j} \rightarrow 0$ has a subsequence converging uniformly to some $\tilde{\varphi}(z, 0)=\tilde{\varphi}^{0} \circ \gamma^{0}(z)$ on $\partial \Omega$. It is clear that $\tilde{\varphi}^{0}, \varphi^{0}$ satisfy the same equations (17.8) with $\lambda=0$. Therefore, $\tilde{\varphi}^{0}=\varphi^{0}$ and consequently $\varphi^{\lambda} \circ \gamma^{\lambda}$ are continuous in the $L^{p}$ norm.

Step 3 (Estimates in $L^{p}$ and Hölder norms). This step works for a), b). We first consider case a) and derive (7.2) for $\beta>0$. We have $L=K$ or $-K$. It suffices to verify it for $\beta=\alpha$. For $z \in \partial \Omega$, write

$$
d \sigma^{\lambda}\left(z^{\lambda}\right)=a^{\lambda}(z) d \sigma(z), \quad D(z)=\frac{a^{\mu}(z)}{a^{\lambda}(z)} \varphi(z, \mu)-\varphi(z, \lambda)
$$

We set $\lambda=\mu$ in (7.3) and then multiply it by $a^{\mu}(z) / a^{\lambda}(z)$. We subtract the new equation by the original (17.3) and get

$$
D(z)+\int_{\partial \Omega} D(\zeta) L^{\lambda}\left(\zeta^{\lambda}, z^{\lambda}\right) d \sigma^{\lambda}\left(\zeta^{\lambda}\right)=E_{0}(z)-E_{1}(z)-E_{2}(z)
$$

with

$$
\begin{gathered}
E_{0}(z)=\frac{a^{\mu}(z)}{a^{\lambda}(z)} \psi_{0}(z, \mu)-\psi_{0}(z, \lambda), \\
E_{1}(z)=\int_{\zeta \in \partial \Omega} \varphi(\zeta, \mu)\left(L^{\mu}\left(\zeta^{\mu}, z^{\mu}\right)-L^{\lambda}\left(\zeta^{\lambda}, z^{\lambda}\right)\right) d \sigma^{\mu}\left(\zeta^{\mu}\right), \\
E_{2}(z)=\left\{\frac{a^{\mu}(z)}{a^{\lambda}(z)}-1\right\} \int_{\zeta \in \partial \Omega} \varphi(\zeta, \mu) L^{\mu}\left(\zeta^{\mu}, z^{\mu}\right) d \sigma^{\mu}\left(\zeta^{\mu}\right)
\end{gathered}
$$


Note that $\left|\frac{a^{\mu}(\cdot)}{a^{\lambda}(\cdot)}-1\right|_{\alpha} \leq C_{1}|\gamma(\cdot, \mu)-\gamma(\cdot, \lambda)|_{1+\alpha}$. We immediately obtain

$$
\left|E_{0}\right|_{\alpha} \leq C_{1}\left(\left|\psi_{0}^{\mu}\right|_{\alpha}|\gamma(\cdot, \mu)-\gamma(\cdot, \lambda)|_{1+\alpha}+\left|\psi_{0}(\cdot, \mu)-\psi_{0}(\cdot, \lambda)\right|_{\alpha}\right) .
$$

By (5.46) with $i=j^{\prime}=0$, we obtain

$$
\left|E_{2}\right|_{\alpha} \leq C|\gamma(\cdot, \mu)-\gamma(\cdot, \lambda)|_{1+\alpha}\left|\varphi^{\mu}\right|_{\alpha}
$$

Define the trivial extension $\tilde{\varphi}_{\mu}^{\lambda}\left(z^{\lambda}\right)=\varphi^{\mu}\left(z^{\mu}\right)$, so it is actually independent of $\lambda$. In particular, since $\varphi(\cdot, \mu) \in \mathcal{B}^{\alpha}$, then $\tilde{\varphi}_{\mu}$ is of class $\mathcal{C}^{\alpha, 0}\left(\partial \Omega_{\gamma}\right)$. Also, define $\tilde{a}_{\mu}^{\lambda}\left(z^{\lambda}\right)=$ $a^{\mu}(z)$ so $\tilde{a}_{\mu} \in \mathcal{C}^{k+\alpha, j}\left(\partial \Omega_{\gamma}\right)$. By Lemma 4.3, for $L=\epsilon K^{*}$ with $\epsilon= \pm 1$,

$$
\epsilon E_{1}(z)=\varphi^{\mu}\left(1-\frac{a^{\mu}(z)}{a^{\lambda}(z)}\right)-2 \operatorname{Re}\left\{\tau^{\mu} \mathcal{C}_{+}^{\mu}\left(\overline{\tau^{\mu}} \tilde{\varphi}_{\mu}^{\mu}\right)\right\}+2 \operatorname{Re}\left\{\tau^{\lambda} \mathcal{C}_{+}^{\lambda}\left(\overline{\tau^{\lambda}} \frac{\tilde{a}_{\mu}^{\lambda}}{a^{\lambda}} \tilde{\varphi}_{\mu}^{\lambda}\right)\right\}
$$

By the Cauchy transform with parameter (Proposition [5.2), we obtain

$$
\left|E_{1}\right|_{\alpha} \leq C\left|\varphi^{\mu}\right|_{\alpha}|\gamma(\cdot, \mu)-\gamma(\cdot, \lambda)|_{1+\alpha} .
$$

Applying Proposition 6.1 (ii) to (7.9), we obtain

$$
\begin{aligned}
|D|_{\alpha} \leq & C\left(|D|_{L^{1}}+\left(\left|\varphi^{\mu}\right|_{\alpha}+\left|\psi_{0}^{\mu}\right|_{\alpha}\right)|\gamma(\cdot, \mu)-\gamma(\cdot, \lambda)|_{1+\alpha}+\left|\psi_{0}(\cdot, \mu)-\psi_{0}(\cdot, \lambda)\right|_{\alpha}\right) \\
\leq & C\left(|\varphi(\cdot, \mu)-\varphi(\cdot, \lambda)|_{L^{1}}+\left(\left|\varphi^{\mu}\right|_{L^{1}}+\left|\psi_{0}^{\mu}\right|_{\alpha}\right)|\gamma(\cdot, \mu)-\gamma(\cdot, \lambda)|_{1+\alpha}\right. \\
& \left.+\left|\psi_{0}(\cdot, \mu)-\psi_{0}(\cdot, \lambda)\right|_{\alpha}\right) .
\end{aligned}
$$

Here the last inequality is obtained by the definition of $D$ and (7.7). The proof of (7.2) is complete when $\beta>0$.

To verify (7.1) for case a), we start with (7.10) and get

$$
\left|E_{0}\right|_{L^{p}} \leq C\left(\left|\psi_{0}(\cdot, \lambda)\right|_{L^{p}}\left|\gamma^{\mu}-\gamma^{\lambda}\right|_{1}+\left|\psi_{0}(\cdot, \mu)-\psi_{0}(\cdot, \lambda)\right|_{L^{p}}\right)
$$

By (5.28), we obtain

$$
\left|L^{\mu}\left(\zeta^{\mu}, z^{\mu}\right)-L^{\lambda}\left(\zeta^{\lambda}, z^{\lambda}\right)\right| \leq C\left|\gamma^{\mu}-\gamma^{\lambda}\right|_{1+\alpha}|\zeta-z|^{\alpha-1} .
$$

By Hölder inequality and Fubini's theorem (or Young's inequality), we have $\left|E_{1}\right|_{L^{p}}$ $\leq C\left|\varphi^{\mu}\right|_{L^{p}}\left|\gamma^{\mu}-\gamma^{\lambda}\right|_{1+\alpha}$. Also, $\left|E_{2}\right|_{L^{p}} \leq C\left(\left|\varphi^{\mu}\right|_{L^{p}}\right)\left|\gamma^{\mu}-\gamma^{\lambda}\right|_{1+\alpha}$. By Proposition 6.1 (ii), we have $\left|\varphi^{\mu}\right|_{L^{p}} \leq C\left(\left|\varphi^{\mu}\right|_{L^{1}}+\left|\psi_{0}^{\mu}\right|_{L^{p}}\right)$. Thus,

$$
\left|\left(E_{0}, E_{1}, E_{2}\right)\right|_{L^{p}} \leq C\left(\left|\psi_{0}(\cdot, \mu)-\psi_{0}(\cdot, \lambda)\right|_{L^{p}}+\left(\left|\varphi^{\lambda}\right|_{L^{1}}+\left|\psi_{0}^{\lambda}\right|_{L^{p}}\right)\left|\gamma^{\mu}-\gamma^{\lambda}\right|_{1+\alpha}\right) .
$$

By (7.9) and Proposition 6.1 (ii) again, we get (7.1). Note that (7.1) for the $L^{\infty}$ case gives us (7.2) for $\beta=0$.

For b), the above arguments are still valid for (7.1)-(7.2) after minor changes. Formula (7.13) for $E_{1}$ needs to be changed when $L=K$ or $-K$ (see Lemma 4.3). The use of Cauchy transform with parameter is still valid, and the same estimate for $E_{1}$ holds. The proof for (ii) and (iii) is complete.

Remark 7.2. The norms of $\psi_{1}, \ldots, \psi_{n}$ do not appear in (7.1)-(7.2). However, when we use (7.1)-(7.2), we need $\varphi^{\lambda}$ to have bounded $L^{1}$ norms at least. The boundedness is established via Lemma 7.1(i), so restrictions on $\psi_{i}$ for $i>0$ enter. 
We want to use (7.9)-(7.12) to compute the derivatives in parameter. Define

$$
\begin{gathered}
\tilde{\partial}_{\lambda} \varphi^{\lambda}\left(z^{\lambda}\right) \stackrel{\text { def }}{=} \partial_{\lambda}\left\{\varphi^{\lambda}\left(z^{\lambda}\right)\right\}+\varphi^{\lambda}\left(z^{\lambda}\right) \partial_{\lambda} \log \left|\partial_{\tau_{z}} \gamma^{\lambda}\right| \\
\mathcal{L}_{1}^{\lambda *} \varphi(z)=\int_{\partial \Omega^{\lambda}} \varphi^{\lambda}\left(\zeta^{\lambda}\right) \partial_{\lambda}\left\{L^{\lambda}\left(\zeta^{\lambda}, z^{\lambda}\right)\right\} d \sigma^{\lambda}\left(\zeta^{\lambda}\right) \\
\mathcal{L}_{2}^{\lambda *} \varphi(z)=\left(\partial_{\lambda} \log \left|\partial_{\tau_{z}} \gamma^{\lambda}\right|\right) \int_{\partial \Omega^{\lambda}} \varphi^{\lambda}(\zeta) L^{\lambda}\left(\zeta^{\lambda}, z^{\lambda}\right) d \sigma^{\lambda}\left(\zeta^{\lambda}\right), \\
\mathcal{L}_{1}^{\lambda} \varphi(z)=\int_{\partial \Omega^{\lambda}} \varphi^{\lambda}\left(\zeta^{\lambda}\right) \partial_{\lambda}\left\{L^{\lambda}\left(z^{\lambda}, \zeta^{\lambda}\right)\right\} d \sigma^{\lambda}\left(\zeta^{\lambda}\right), \\
\mathcal{L}_{2}^{\lambda} \varphi(z)=\left(\partial_{\lambda} \log \left|\partial_{\tau_{z}} \gamma^{\lambda}\right|\right) \int_{\partial \Omega^{\lambda}} \varphi^{\lambda}(\zeta) L^{\lambda}\left(z^{\lambda}, \zeta^{\lambda}\right) d \sigma^{\lambda}\left(\zeta^{\lambda}\right) .
\end{gathered}
$$

Set $\mathcal{L}_{i}^{*} \varphi=\left\{\mathcal{L}_{i}^{\lambda *} \varphi\right\}$. Let $\ell_{1}, \ldots, \ell_{n}$ be the standard basis of $\operatorname{ker}(I+\mathcal{L})$.

Lemma 7.3. Let $\gamma^{\lambda}$ embed $\partial \Omega$ onto $\partial \Omega^{\lambda}$ with $\gamma \in \mathcal{B}^{2+\alpha, 1}(\partial \Omega)$.

a) Keep the assumptions in a) of Lemma 7.1. Assume further that $\psi_{0} \in$ $\mathcal{C}_{*}^{0,1}(\partial \Omega)$ and $\psi_{i} \in \mathcal{C}^{1}([0,1])$ for $i>0$. Then $\varphi \in \mathcal{C}_{*}^{0,1}(\partial \Omega)$ and

$$
\begin{gathered}
\tilde{\partial}_{\lambda} \varphi^{\lambda}+\left(\mathcal{L}^{\lambda}\right)^{*} \tilde{\partial}_{\lambda} \varphi^{\lambda}=\tilde{\partial}_{\lambda} \psi_{0}^{\lambda}-\left(\mathcal{L}_{1}^{\lambda *}+\mathcal{L}_{2}^{\lambda *}\right) \varphi^{\lambda}, \\
\int_{\partial \Omega^{\lambda}}\left(\tilde{\partial}_{\lambda} \varphi^{\lambda}\right) \ell_{i}^{\lambda} d \sigma^{\lambda}=\partial_{\lambda} \psi_{i}^{\lambda} .
\end{gathered}
$$

b) Keep the assumptions in b) of Lemma 7.1. Assume further that $\psi_{0} \in$ $\mathcal{C}_{*}^{0,1}(\partial \Omega)$ and $\psi_{i} \in \mathcal{C}^{1}([0,1])$. Then $\varphi \in \mathcal{C}_{*}^{0,1}(\partial \Omega)$ and

$$
\begin{gathered}
\tilde{\partial}_{\lambda} \varphi^{\lambda}+\mathcal{L}^{\lambda} \tilde{\partial}_{\lambda} \varphi^{\lambda}=\tilde{\partial}_{\lambda} \psi^{\lambda}-\left(\mathcal{L}_{1}^{\lambda}+\mathcal{L}_{2}^{\lambda}\right) \varphi^{\lambda}, \\
\int_{\partial \Omega^{\lambda}}\left(\tilde{\partial}_{\lambda} \varphi^{\lambda}\right) \ell_{i}^{\lambda} d \sigma^{\lambda}=\partial_{\lambda} \psi_{i}^{\lambda}-\int_{\partial \Omega^{\lambda}} \varphi^{\lambda}\left(\partial_{\lambda} \ell_{i}^{\lambda}\right) d \sigma^{\lambda}, \quad 1 \leq i \leq n .
\end{gathered}
$$

c) Let $0 \leq \beta \leq \alpha$. In a) and b) of Lemma 7.1, we have $\varphi \in \mathcal{B}_{*}^{\beta, j}\left(\partial \Omega_{\gamma}\right)$ provided $\psi_{0} \in \mathcal{B}_{*}^{\beta, j}\left(\partial \Omega_{\gamma}\right), \psi_{i} \in \mathcal{C}^{j}([0,1])$, and $\gamma \in \mathcal{B}^{j+1+\beta, j}(\partial \Omega)$; the same assertion holds if $\mathcal{C}$ substitutes for $\mathcal{B}$.

Proof. For simplicity we express $\varphi \in \mathcal{B}^{\bullet}\left(\partial \Omega_{\gamma}\right)$ as $\varphi \in \mathcal{B}^{\bullet}$, and $\gamma \in \mathcal{B}^{\bullet}(\partial \Omega)$ as $\gamma \in \mathcal{B}^{\bullet}$, etc.

a) Let us recall some identities in the previous proof. Fix $\lambda$. Recall that $\ell_{i}$ are locally constant when $L=K$ or $-K$. We also use the notation $f^{\lambda}\left(z^{\lambda}\right)=f(z, \lambda)$. By (7.9)-(7.12), the difference quotient

$$
\hat{D}(z, \mu)=\frac{1}{\mu-\lambda}\left(\frac{a^{\mu}(z)}{a^{\lambda}(z)} \varphi(z, \mu)-\varphi(z, \lambda)\right)
$$

satisfies

$$
\begin{gathered}
\hat{D}(z, \mu)+\int_{\partial \Omega} \hat{D}(\zeta, \mu) L^{\lambda}\left(\zeta^{\lambda}, z^{\lambda}\right) d \sigma^{\lambda}\left(\zeta^{\lambda}\right)=\hat{E}_{0}(z, \mu)-\hat{E}_{1}(z, \mu)-\hat{E}_{2}(z, \mu), \\
\int_{\zeta \in \partial \Omega} \hat{D}(\zeta, \mu) \ell_{i}^{\lambda} d \sigma^{\lambda}\left(\zeta^{\lambda}\right)=\frac{1}{\mu-\lambda}\left(\psi_{i}^{\mu}-\psi_{i}^{\lambda}\right), \quad 1 \leq i \leq n
\end{gathered}
$$


with

$$
\begin{gathered}
\hat{E}_{0}(z, \mu)=\frac{1}{\mu-\lambda}\left(\frac{a^{\mu}(z)}{a^{\lambda}(z)} \psi_{0}(z, \mu)-\psi_{0}(z, \lambda)\right) \\
\hat{E}_{1}(z, \mu)=\int_{\zeta \in \partial \Omega} \varphi(\zeta, \mu) \frac{L^{\mu}\left(\zeta^{\mu}, z^{\mu}\right)-L^{\lambda}\left(\zeta^{\lambda}, z^{\lambda}\right)}{\mu-\lambda} d \sigma^{\mu}\left(\zeta^{\mu}\right) \\
\hat{E}_{2}(z, \mu)=\frac{a^{\mu}(z)-a^{\lambda}(z)}{a^{\lambda}(z)(\mu-\lambda)} \int_{\zeta \in \partial \Omega} \varphi(\zeta, \mu) L^{\mu}\left(\zeta^{\mu}, z^{\mu}\right) d \sigma^{\mu}\left(\zeta^{\mu}\right) .
\end{gathered}
$$

As $\mu \rightarrow \lambda$, it is clear that $\hat{\psi}_{0}(z, \mu)$ converges uniformly to $\tilde{\partial}_{\lambda} \psi_{0}^{\lambda}$. We want to show that as $\mu$ tends to $\lambda, \hat{D}(z, \mu)$ converges uniformly to a continuous function. Then the existence of the limit function implies that $\partial_{\lambda} \varphi^{\lambda}$ exists and the limit function must be $\tilde{\partial}_{\lambda} \varphi^{\lambda}$.

By Lemma 7.1. $\varphi \in \mathcal{C}^{0,0}$. It is easy to see that $\hat{E}_{1}, \hat{E}_{2}$ are continuous at $\mu \neq \lambda$. Also, $\hat{E}_{2}(z, \mu)$ converges uniformly to $\left(\mathcal{L}_{2}^{\lambda *} \phi\right)\left(z^{\lambda}\right)$ as $\mu \rightarrow \lambda$; in particular, $\left\{\hat{E}_{2}^{\mu}\right\}$ extends to an element in $\mathcal{C}^{0,0}$. For $\zeta \neq z$, by the mean-value theorem and (5.26) we obtain

$$
\left|\frac{K^{\mu}\left(\zeta^{\mu}, z^{\mu}\right)-K^{\lambda}\left(\zeta^{\lambda}, z^{\lambda}\right)}{\mu-\lambda}\right| \leq C|\zeta-z|^{\alpha-1} .
$$

Thus $\hat{E}_{1}(z, \mu)$ converges uniformly to $\mathcal{L}_{1}^{\lambda *} \varphi\left(z^{\lambda}\right)$ as $\mu \rightarrow \lambda$, and $\left\{\hat{E}_{1}^{\mu} ; \mu \neq \lambda\right\}$ extends to an element in $\mathcal{C}^{0,0}$. By Lemma 7.1 (ii) with $\beta=0$, we conclude that as $\mu \rightarrow \lambda$, $\hat{D}(\cdot, \mu)$ has a limit $\tilde{\partial}_{\lambda} \varphi^{\lambda}$ satisfying (7.15)-(7.16).

b) By a), $\phi_{0}, \ldots, \phi_{m}$ are of class $\mathcal{C}^{1,1}$ when $\gamma \in \mathcal{B}^{2+\alpha, 1}$. Thus, in all cases, we have $\ell_{i} \in \mathcal{C}^{1,1}$. Fix $\lambda$. We need some minor changes in the above arguments. The difference quotient $\hat{D}(z, \mu)$ satisfies

$$
\begin{gathered}
\hat{D}(z, \mu)+\int_{\partial \Omega} \hat{D}(\zeta, \mu) L^{\lambda}\left(z^{\lambda}, \zeta^{\lambda}\right) d \sigma^{\lambda}\left(\zeta^{\lambda}\right)=\hat{E}_{0}(z, \mu)-\hat{E}_{1}^{*}(z, \mu)-\hat{E}_{2}^{*}(z, \mu), \\
\int_{\zeta \in \partial \Omega} \hat{D}(\zeta, \mu) \ell_{i}^{\lambda} d \sigma^{\lambda}\left(\zeta^{\lambda}\right)=\frac{\psi_{i}^{\mu}-\psi_{i}^{\lambda}}{\mu-\lambda}-\int_{\zeta \in \partial \Omega} \varphi(\zeta, \mu) \frac{\ell_{i}^{\mu}\left(\zeta^{\mu}\right)-\ell_{i}^{\lambda}\left(\zeta^{\lambda}\right)}{\mu-\lambda} d \sigma^{\mu}\left(\zeta^{\mu}\right)
\end{gathered}
$$

with

$$
\begin{aligned}
& \hat{E}_{1}^{*}(z, \mu)=\int_{\zeta \in \partial \Omega} \varphi(\zeta, \mu) \frac{L^{\mu}\left(z^{\mu}, \zeta^{\mu}\right)-L^{\lambda}\left(z^{\lambda}, \zeta^{\lambda}\right)}{\mu-\lambda} d \sigma^{\mu}\left(\zeta^{\mu}\right), \\
& \hat{E}_{2}^{*}(z, \mu)=\frac{a^{\mu}(z)-a^{\lambda}(z)}{a^{\lambda}(z)(\mu-\lambda)} \int_{\zeta \in \partial \Omega} \varphi(\zeta, \mu) L^{\mu}\left(z^{\mu}, \zeta^{\mu}\right) d \sigma^{\mu}\left(\zeta^{\mu}\right) .
\end{aligned}
$$

By Lemma 7.1, $\varphi$ is continuous. It is easy to see that $\hat{E}_{1}^{*}, \hat{E}_{2}^{*}$ are continuous at $\mu \neq \lambda$. Also, $\left(\hat{E}_{2}^{*}\right)^{\mu}\left(z^{\mu}\right) \stackrel{\text { def }}{=} \hat{E}_{2}^{*}(z, \mu)$ converges uniformly to $\mathcal{L}_{2}^{\lambda} \varphi\left(z^{\lambda}\right)$ as $\mu \rightarrow \lambda$, and $\left\{\left(E_{2}^{*}\right)^{\mu} ; \mu \neq \lambda\right\}$ extends to an element in $\mathcal{C}^{0,0}$. In addition, $\hat{E}_{1}^{*}(z, \mu)$ converges to $\mathcal{L}_{1}^{\lambda} \varphi\left(z^{\lambda}\right)$ as $\mu \rightarrow \lambda$, and $\left\{\left(E_{1}^{*}\right)^{\mu} ; \mu \neq \lambda\right\}$ extends to an element in $\mathcal{C}^{0,0}$. By Lemma 7.1 (ii) with $\beta=0$, we conclude that as $\mu \rightarrow \lambda, \hat{D}(z, \mu)$ converges uniformly to a limit function, which is denoted by $\left(\tilde{\partial}_{\lambda} \varphi^{\lambda}\right)\left(z^{\lambda}\right)$ and satisfies (7.17)-(7.18).

c) When $j=0$ we get $\varphi \in \mathcal{B}^{\beta, 0}$ from Lemma 7.1 (i) and (ii) and Proposition 6.1 (ii) and (iii). We further have $\varphi \in \mathcal{C}^{\beta, 0}$ for $\psi_{0} \in \mathcal{C}^{\beta, 0}$ and $\gamma \in \mathcal{C}^{1+\alpha, 0}$. Assume that the assertions hold when $j$ is replaced by $j-1$. Thus $\varphi \in \mathcal{B}_{*}^{\beta, j-1}$. We first consider case a). Then, we have (7.15)-(7.16). By (5.46) with $j^{\prime}=j$, we know that $\mathcal{L}_{1}^{*} \varphi$ and $\mathcal{L}_{2}^{*} \varphi$ are in $\mathcal{B}_{*}^{\beta, j-1}$. Also $\left\{\tilde{\partial}_{\lambda} \psi_{0}^{\lambda}\right\}$ is in $\mathcal{B}_{*}^{\beta, j-1}$ and $\left\{\partial_{\lambda} \psi_{i}^{\lambda}\right\}$ are in $\mathcal{C}^{j-1}([(0,1])$ 
for $i>0$. By the induction hypothesis, we conclude that $\left\{\tilde{\partial}_{\lambda} \varphi^{\lambda}\right\} \in \mathcal{B}_{*}^{\beta, j-1}$. Hence $\left\{\partial_{\lambda} \varphi^{\lambda}\right\} \in \mathcal{B}_{*}^{\beta, j-1}$. Combining with $\varphi \in \mathcal{B}^{\beta, 0}$, we get $\varphi \in \mathcal{B}_{*}^{\beta, j}$. We can also verify that $\varphi \in \mathcal{C}_{*}^{\beta, j}$ by Lemma 7.1 (iii) and (5.47), when $\psi \in \mathcal{C}_{*}^{\beta, j}$ and $\gamma \in \mathcal{C}^{j+1+\alpha, j}$.

For case b), we first apply results from a) and conclude that $\phi_{0}, \ldots, \phi_{m}$ are in $\mathcal{B}_{*}^{\alpha, j}$. This shows that $\left\{\int_{\partial \Omega^{\lambda}} \varphi^{\lambda} \phi_{i}^{\lambda} d \sigma^{\lambda}\right\}$ are in $\mathcal{C}^{j-1}([0,1])$ if $\varphi \in \mathcal{C}_{*}^{0, j-1}$. We substitute (7.17)-(7.18) for (7.15)-(7.16) and substitute (5.44)-(5.45) for (5.46)-(5.47) with $j^{\prime}=j$. With minor changes in the arguments, we verify the assertions for b) too.

By Proposition 6.1 (iv) and Corollary 6.4, we have proved the required regularity in higher-order derivatives of solutions to the integral equations for a fixed parameter. We are ready to study the regularity of higher-order derivatives for the parameter case.

Proposition 7.4. Let $\gamma^{\lambda}$ embed $\partial \Omega$ onto $\partial \Omega^{\lambda}$. Let $k \geq j \geq 0$ and $0 \leq \beta \leq \alpha<1$. Let $\beta>0$ when $k>0$. Suppose that $\gamma \in \mathcal{B}^{k+1+\alpha, j}(\partial \Omega), \psi_{0} \in \mathcal{B}^{k+\beta, j}\left(\partial \Omega_{\gamma}\right)$, $\psi_{i} \in \mathcal{C}^{j}([0,1])$ for $1 \leq i \leq n$, and $\varphi^{\lambda} \in L^{1}\left(\partial \Omega^{\lambda}\right)$.

a) Let $\left\{\mathcal{L}^{\lambda}\right\}$ be $\left\{\mathcal{K}^{\lambda}\right\}$ or $\left\{-\mathcal{K}^{\lambda}\right\}$. Suppose that

$$
\varphi^{\lambda}+\left(\mathcal{L}^{\lambda}\right)^{*} \varphi^{\lambda}=\psi_{0}^{\lambda}, \quad\left\langle\varphi^{\lambda}, \ell_{j}^{\lambda}\right\rangle=\psi_{i}^{\lambda}, \quad 1 \leq i \leq n .
$$

Then $\varphi \in \mathcal{B}^{k+\beta, j}\left(\partial \Omega_{\gamma}\right)$.

b) Let $\left\{\mathcal{L}^{\lambda}\right\}$ be $\left\{\left(\mathcal{K}^{\lambda}\right)^{*}\right\}$ or $\left\{-\left(\mathcal{K}^{\lambda}\right)^{*}\right\}$. Suppose that

$$
\varphi^{\lambda}+\mathcal{L}^{\lambda} \varphi^{\lambda}=\psi_{0}^{\lambda}, \quad\left\langle\varphi^{\lambda}, \ell_{j}^{\lambda}\right\rangle=\psi_{i}^{\lambda}, \quad 1 \leq i \leq n .
$$

Then $\varphi \in \mathcal{B}^{k+\beta, j}\left(\partial \Omega_{\gamma}\right)$.

c) Let $l \leq k+1$ and $\beta>0$ when $l>0$. Let $\left\{\mathcal{L}^{\lambda}\right\}$ be $\left\{\mathcal{K}^{\lambda}\right\}$ or $\left\{-\mathcal{K}^{\lambda}\right\}$. Suppose that $\psi_{0} \in \mathcal{B}^{l+\beta, j}\left(\partial \Omega_{\gamma}\right)$. Then the solution $\varphi$ to (7.19) is in $\mathcal{B}^{l+\beta, j}\left(\partial \Omega_{\gamma}\right)$.

a), b) and c) remain true if the symbol $\mathcal{C}^{\bullet}$ substitutes for $\mathcal{B}^{\bullet}$ in all conditions and assertions.

Proof. To simplify notation, we express $\varphi \in \mathcal{B}^{\bullet}\left(\partial \Omega_{\gamma}\right)$ as $\varphi \in \mathcal{B}^{\bullet}$, and $\gamma \in \mathcal{B}^{\bullet}(\partial \Omega)$ as $\gamma \in \mathcal{B}^{\bullet}$, etc. The proposition is proved when $k=0$ and $\beta=0$, by Proposition 6.1 (ii) and Lemma 7.1 (i) and (ii). We may assume that $\beta=\alpha$.

a) We first verify the assertions when $j=0$. When $k=0$ we get $\varphi \in \mathcal{B}^{\beta, 0}$ by Proposition 6.1 (ii) and $\varphi \in \mathcal{C}^{\beta, 0}$ by Lemma 7.1 (i) and (iii). We apply Proposition 5.5. Then (5.46) implies $\varphi \in \mathcal{B}^{k+\beta, 0}$ for $\gamma \in \mathcal{B}^{k+1+\alpha, 0}$; (5.47) implies $\varphi \in \mathcal{C}^{k+\beta, 0}$ for $\gamma \in \mathcal{C}^{k+1+\alpha, 0}$ and $\psi_{0} \in \mathcal{C}^{k+\alpha, 0}$.

For $j>0$, assume that a) is valid when $j$ is replaced by $j-1$. Thus, $\varphi \in \mathcal{B}^{k+\beta, j-1}$. Also, $\varphi \in \mathcal{C}^{k+\beta, j-1}$ for $\gamma \in \mathcal{C}^{k+1+\alpha, j-1}$ and $\psi_{0} \in \mathcal{C}^{k+\alpha, j-1}$. Since $\psi_{0} \in \mathcal{C}^{1,1}$ and $\psi_{i} \in \mathcal{C}^{1}([0,1])$ for $i>0$, Lemma 7.3 implies that

$$
\tilde{\partial}_{\lambda} \varphi^{\lambda}+\left(\mathcal{L}^{\lambda}\right)^{*} \tilde{\partial}_{\lambda} \varphi^{\lambda}=\tilde{\partial}_{\lambda} \psi_{0}^{\lambda}-\left(\mathcal{L}_{1}^{\lambda *}+\mathcal{L}_{2}^{\lambda *}\right) \varphi^{\lambda}, \quad\left\langle\tilde{\partial}_{\lambda} \varphi^{\lambda}, \ell_{i}^{\lambda}\right\rangle=\partial_{\lambda} \psi_{i}^{\lambda}, \quad i>0 .
$$

Here $\left\{\partial_{\lambda} \psi_{i}\right\} \in \mathcal{C}^{j-1}$. Also, $\tilde{\partial}_{\lambda} \psi_{0} \in \mathcal{B}^{k-1+\alpha, j-1}$ by

$$
\tilde{\partial}_{\lambda} \psi_{0}^{\lambda}\left(z^{\lambda}\right)=\partial_{\lambda} \psi_{0}^{\lambda}\left(z^{\lambda}\right)+\psi_{0}^{\lambda}\left(z^{\lambda}\right) \partial_{\lambda} \log \left|\partial_{\tau} \gamma^{\lambda}\right|, \quad\left\{\partial_{\lambda} \log \left|\partial_{\tau} \gamma^{\lambda}\right|\right\} \in \mathcal{B}^{k-1+\alpha, j-1} .
$$


Combining $\varphi \in \mathcal{B}^{k+\beta, j-1} \subset \mathcal{B}^{k-1+\beta, j-1}$ with

$$
\begin{gathered}
\mathcal{L}_{1}^{\lambda *} \varphi(z)=\int_{\partial \Omega^{\lambda}} \varphi^{\lambda}(\zeta) \partial_{\lambda}\left\{L^{\lambda}\left(\zeta^{\lambda}, z^{\lambda}\right)\right\} d \sigma^{\lambda}\left(\zeta^{\lambda}\right) \\
\mathcal{L}_{2}^{\lambda *} \varphi(z)=\left(\partial_{\lambda} \log \left|\partial_{\tau} \gamma^{\lambda}(z)\right|\right) \int_{\partial \Omega^{\lambda}} \varphi^{\lambda}(\zeta) L^{\lambda}\left(\zeta^{\lambda}, z^{\lambda}\right) d \sigma^{\lambda}\left(\zeta^{\lambda}\right),
\end{gathered}
$$

we see from (5.46) that $\mathcal{L}_{i}^{*} \varphi \in \mathcal{B}^{k-1+\alpha, j-1}$. Thus $\left\{\tilde{\partial}_{\lambda} \varphi^{\lambda}\right\}$ and $\left\{\partial_{\lambda} \varphi^{\lambda}\right\}$ are in $\mathcal{B}^{k-1+\alpha, j-1}$. Combining with $\left\{\partial_{\lambda} \varphi^{\lambda}\right\} \in \mathcal{B}^{k+\alpha, 0}$, we see that $\left\{\varphi^{\lambda}\right\}$ is in $\mathcal{B}^{k+\alpha, j}$. To verify $\varphi \in \mathcal{C}^{k+\alpha, j}$ for $\gamma \in \mathcal{C}^{k+1+\alpha, j}$, we use (5.47) instead of (5.46).

b) Note that the case where $k=l=0$ is established by Lemma 7.1 So we assume that $k \geq 1$. Although we are dealing with the same integral equations as in a), i.e. $\varphi^{\lambda} \pm\left(\mathcal{K}^{\lambda}\right)^{*} \varphi^{\lambda}=\psi_{0}^{\lambda}$, the functions $\ell_{i}$ which appeared in $\left\langle\varphi^{\lambda}, \ell_{i}^{\lambda}\right\rangle=\psi_{i}^{\lambda}$ are no longer constants in general. Nevertheless, a) implies that $\phi_{0}, \phi_{1}, \ldots, \phi_{m}$ are of class $\mathcal{B}^{k+\alpha, j}$ or are of class $\mathcal{C}^{k+\alpha, j}$ when $\gamma \in \mathcal{C}^{k+1+\alpha, j}$. In any case, we have $\ell_{i} \in \mathcal{B}^{k+\alpha, j}$. Then

$$
\int_{\partial \Omega^{\lambda}}\left(\tilde{\partial}_{\lambda} \varphi^{\lambda}\right) \ell_{i}^{\lambda} d \sigma^{\lambda}=\partial_{\lambda} \psi_{i}^{\lambda}-\int_{\partial \Omega^{\lambda}} \varphi^{\lambda}\left(\partial_{\lambda} \ell_{i}^{\lambda}\right) d \sigma^{\lambda}
$$

are in $\mathcal{C}^{j-1}([0,1])$, if we know $\psi_{i} \in \mathcal{C}^{j}$ and $\varphi \in \mathcal{C}_{*}^{0, j-1}$. The latter is ensured if $\varphi \in \mathcal{B}^{k-1+\alpha, j-1}$ with $k \geq j$ and $j \geq 1$. Then, $\mathcal{L}_{i}\left(\mathcal{B}^{k-1+\alpha, j-1}\right)$ is contained in $\mathcal{B}^{k-1+\alpha, j-1}$ by (5.46) and

$$
\begin{gathered}
\mathcal{L}_{1}^{\lambda} \varphi(z)=\int_{\partial \Omega^{\lambda}} \varphi^{\lambda}\left(\zeta^{\lambda}\right) \partial_{\lambda}\left\{L^{\lambda}\left(z^{\lambda}, \zeta^{\lambda}\right)\right\} d \sigma^{\lambda}\left(\zeta^{\lambda}\right) \\
\mathcal{L}_{2}^{\lambda} \varphi(z)=\left(\partial_{\lambda} \log \left|\partial_{\tau} \gamma^{\lambda}(z)\right|\right) \int_{\partial \Omega^{\lambda}} \varphi^{\lambda}(\zeta) L^{\lambda}\left(z^{\lambda}, \zeta^{\lambda}\right) d \sigma^{\lambda}\left(\zeta^{\lambda}\right) .
\end{gathered}
$$

Finally, $\left\{\partial_{\lambda} \log \left|\partial_{\tau} \gamma^{\lambda}\right|\right\}$ is in $\mathcal{B}^{k-1+\alpha, j-1}$, which implies that if $\left\{\tilde{\partial}_{\lambda} \varphi^{\lambda}\right\} \in \mathcal{B}^{l-1+\alpha, j-1}$, then $\left\{\partial_{\lambda} \varphi^{\lambda}\right\}$ remains in $\mathcal{B}^{l-1+\alpha, j-1}$. With these observations, the induction proof in a) is valid without essential changes. To verify $\varphi \in \mathcal{C}^{k+\alpha, j}$ when $\gamma \in \mathcal{C}^{k+1+\alpha, j}$ and $\psi_{0} \in \mathcal{C}^{k+\alpha, j}$, we use (5.47) instead of (5.46).

c) To show $\varphi \in \mathcal{B}^{k+1+\alpha, j}$, we cannot use the induction proof in b) when $\psi_{i} \in$ $\mathcal{B}^{k+1+\alpha, j}$. For that $\left\{\tilde{\partial}_{\lambda} \varphi^{\lambda}\right\} \in \mathcal{B}^{k+\alpha, j-1}$, defined by (7.14), does not imply that $\left\{\partial_{\lambda} \varphi^{\lambda}\right\}$ is in $\mathcal{B}^{k+\alpha, j-1}$.

Instead, we apply induction on $l$. If $l=0$, by Lemma $7.3 \mathrm{c}$ ) we get $\varphi \in B_{*}^{\alpha, j}$ and $\varphi \in \mathcal{C}_{*}^{\alpha, j}$ when $\psi_{0} \in \mathcal{C}_{*}^{\alpha, j}$ and $\gamma \in \mathcal{C}^{j+1+\alpha, j}$. In particular, c) is valid when $l=0$. Assume that $\mathrm{c}$ ) is valid when $l$ with $>0$ is replaced by $l-1$. We have

$$
\partial_{\tau^{\lambda}} \varphi^{\lambda}-\left(\mathcal{L}^{\lambda}\right)^{*} \partial_{\tau^{\lambda}} \varphi^{\lambda}=\partial_{\tau^{\lambda}} \psi_{0}^{\lambda}, \quad \int_{\gamma_{i}} \partial_{\tau^{\lambda}} \varphi^{\lambda} d \sigma^{\lambda}=0, \quad i \geq 0 .
$$

Note that $\left\{\partial_{\tau^{\lambda}} \psi_{0}^{\lambda}\right\}$ is in $\mathcal{B}^{l-1+\alpha, j}$ when $l-1 \geq j$ and it is in $\mathcal{B}^{j-1+\alpha, j-1}$ when $l=j$. By b), we conclude that $\partial_{\tau} \varphi \in \mathcal{B}^{l-1+\alpha, j}$ for $k \geq l-1 \geq j$ or in $\mathcal{B}^{j-1+\alpha, j-1}$ when $l=j$. Combining with $\varphi \in \mathcal{B}_{*}^{\alpha, j}$, we conclude that $\varphi \in \mathcal{B}^{l+\alpha, j}$. We can also verify that $\varphi \in \mathcal{C}^{l+\alpha, j}$ when $\psi_{0} \in \mathcal{C}^{l+\alpha, j}$ and $\gamma \in \mathcal{C}^{k+1+\alpha, j}$.

One can give another proof for c) by using Lemma 6.3 and a), which avoids the induction argument. We leave the details to the reader. 


\section{HÖLDER SPACES FOR EXTERIOR DOMAINS WITH PARAMETER}

In this section, we return to the definition of Hölder spaces with parameter. However, the reader can turn to the proof of Theorem 9.2 for interior domains by skipping this section. Lemma 8.2 shows that elements in $\mathcal{B}^{k+\beta, j}\left(\partial \Omega_{\gamma}\right)$ extend to elements in $\mathcal{B}^{k+\beta, j}\left(\bar{\Omega}_{\Gamma}\right)$. Lemma 8.6 shows that possibly by restricting $\lambda$ to a subinterval, we can extend a family of embeddings $\gamma^{\lambda}$ of $\partial \Omega$ with $\gamma \in \mathcal{B}^{k+\beta, j}(\partial \Omega) \cap$ $\mathcal{C}^{1,0}(\partial \Omega)$ to a family of embeddings $\Gamma^{\lambda}$ of $\bar{\Omega}$ with $\Gamma \in \mathcal{B}^{k+\beta, j}(\bar{\Omega}) \cap \mathcal{C}^{1,0}(\bar{\Omega})$. The two lemmas and Lemma 2.2 form basic properties of Hölder spaces for interior domains with parameter. We also define Hölder spaces for exterior domains with parameter. Finally, we extend estimates on Cauchy transforms and single and double layer potentials to exterior domains for our Hölder spaces. To extend families of finitely smooth embeddings from $\partial \Omega$ into $\bar{\Omega}$, we apply a type of Whitney extension with parameter. However, the real analytic extension is more subtle, for which we need the real analyticity of solutions to the Dirichlet problem with real analytic parameter. The connection between extensions of functions and solutions of the Dirichlet problem was observed by Whitney [18]. When an exterior domain $\Omega^{\prime}=$ $\overline{\mathbf{C}} \backslash \bar{\Omega}$ is considered, we assume without loss of generality that $\Omega$ is simply connected.

Lemma 8.1. Let $J, K$ be non-negative integers or $\infty$, and let $0 \leq \beta<1$. For $0 \leq k<K$, let $\epsilon_{k+1}$ be decreasing positive numbers and $0 \leq j_{k}<J+1$ be nondecreasing integers. Suppose that $j_{k}=J$ for some $k$ if $J<\infty$ and $j_{k}$ tends to $J$ if $J$ and $K$ are infinite. Let $\Omega$ be a bounded domain with $\partial \Omega \in \mathcal{C}^{K+\beta} \cap \mathcal{C}^{1}$. Suppose that $f_{i} \in \mathcal{B}_{*}^{K-i+\beta, J}(\partial \Omega)$ (resp. $\mathcal{C}_{*}^{K-i+\beta, J}(\partial \Omega)$ ) for $0 \leq i<K+1$. There exists $E f \in \mathcal{B}_{*}^{K+\beta, J}(\bar{\Omega})$ (resp. $\mathcal{C}_{*}^{K+\beta, J}(\bar{\Omega})$ ) satisfying $\partial_{\nu}^{i} E f=f_{i}$ for $0 \leq i<K+1$. Furthermore, Ef has the following properties:

(i) The extension operator $f \rightarrow E f$ depends only on $i, \partial \Omega$ and the upper bound $M_{i}$ of $\epsilon_{i}^{-1}$ and $\left|f_{l}\right|_{i-l+\beta, j_{i-1}}$ for $0 \leq l \leq i$ and $0<i<K+1$. Moreover,

$$
\begin{aligned}
& |E f|_{k+\beta, j_{k}} \leq \epsilon_{k+1}+C_{k}(\epsilon, f) \sum_{i \leq k}\left|f_{i}\right|_{k-i+\beta, j_{k}}, \quad k<K \leq \infty, \\
& |E f|_{K+\beta, j} \leq C_{K}(\epsilon, f) \sum_{i \leq K}\left|f_{i}\right|_{K-i+\beta, j}, \quad K<\infty, \quad 0 \leq j<J+1 .
\end{aligned}
$$

Here $C_{k}(\epsilon, f)$ depends only on $k, \partial \Omega$ and $M_{l}$ for $0<l \leq k$.

(ii) Assume further that $f_{0}$ is constant and $f_{i}$ vanish in a neighborhood $U$ of $p$ in $\partial \Omega \times[0,1]$ for all $i>0$ with $i+J \leq K$. Then $E f$ is constant on some neighborhood $V$ of $p$ in $\bar{\Omega} \times[0,1]$. Moreover, $V$ depends only on $U$.

Proof. We cover $\partial \Omega$ by open subsets $U_{p}$ of $\bar{\Omega}$ and find $\mathcal{C}^{\infty}$ functions $\chi_{p}$ with compact support in $U_{p}$ such that $\sum_{p=1}^{p_{0}} \chi_{p}=1$ on $\partial \Omega$. Here $p_{0}$ is finite. We may assume that there exist an open subset $V_{p}$ of $\bar{\Omega}$, which contains $U_{p}$, and a $\mathcal{C}^{K+\beta} \cap \mathcal{C}^{1}$ diffeomorphism $\psi_{p}$ on $\overline{V_{p}}$ which maps $V_{p}$ onto $V^{*}=(-2,2) \times[0,2)$ and $U_{p}$ onto $U^{*}=(-1,1) \times[0,1)$. We also assume that $\psi_{p}$ sends $\partial_{\nu}$ into $A_{p} \partial_{y}+B_{p} \partial_{x}$ such that $1 / C<\left|A_{p}\right|<C$. Here $A_{p}$ and $B_{p}$ are of class $\mathcal{C}^{K-1+\beta} \cap \mathcal{C}^{0}$ on $\overline{V^{*}}$. It suffices to find $h_{p} \in \mathcal{B}_{*}^{K+\beta, J}(\bar{\Omega})$ such that $\operatorname{supp} h_{p} \subset V_{p} \times[0,1]$ and $\partial_{\nu}^{i} h_{p}=\chi_{p} f_{i}=h_{p i}$ on $\left(V_{p} \cap \partial \Omega\right) \times[0,1]$. Then $E f=\sum h_{p}$ is a desired extension.

We now drop the subscript $p$ in all expressions. In the new coordinates, we still denote $f, h_{i}, \chi$, and $\nu$ by the same symbols. For instance, $h_{i}$ denotes $h_{p i} \circ \psi_{p}^{-1}$. We 
have

$\partial_{\nu}^{i}=A^{i} \partial_{y}^{i}+\sum_{0<m \leq i} \sum_{l \leq m, l<i} B_{i l m} \partial_{x}^{m-l} \partial_{y}^{l}, \quad \partial_{y}^{i}=A^{-i} \partial_{\nu}^{i}+\sum_{0<m \leq i} \sum_{l \leq m, l<i} \tilde{B}_{i l m} \partial_{x}^{m-l} \partial_{\nu}^{l}$.

Note that $B_{i l m}$ and $\tilde{B}_{i l m}$ are in $\mathcal{C}^{K-i+(m-1)+\beta} \cap \mathcal{C}^{0}$ on $\overline{V^{*}}$. To achieve $\partial_{\nu}^{i} h=h_{i}$ on $V^{*}$, we need

$$
\partial_{y}^{i} h=A^{-i} h_{i}+\sum_{0<m \leq i} \sum_{l \leq m, l<i} \tilde{B}_{i l m} \partial_{x}^{m-l} h_{l} .
$$

Changing notation, we write (8.3) as $\partial_{y}^{i} f=f_{i}$. The support of $f_{i}$ is contained in $[-1,1] \times[0,1]$, and $f_{i}$ is in $\mathcal{B}_{*}^{K-i+\beta, J}([-2,2])$. If necessary, we will replace $\epsilon_{k}$ by $\epsilon_{k} / C$ with $C$ depending on the numbers of sets $U_{p}$ and diffeomorphisms $\psi_{p}$.

Fix $0<\delta<1 / 2$. Let $\phi$ be a smooth function on $\mathbf{R}$ with support in $(-\delta, \delta)$ such that $\int_{\mathbf{R}} \phi(y) d y=1$. We first need to replace $y^{i} f_{i}(x, \lambda)$ by $y^{i} g_{i}(x, y, \lambda)$ to achieve the $\mathcal{B}_{*}^{K+\beta, J}$ smoothness; when $K=\infty$, we still need the replacement to estimate the $|\cdot|_{k+\beta, j}$ norm of $y^{i} g_{i}(x, y, \lambda)$ via $\left|f_{i}\right|_{k-i+\beta, j}$. This requires us to correct the $i$-th $y$-derivative of $y^{i} g_{i}(x, y, \lambda)$ due to the presence of $y^{i_{1}} g_{i_{1}}(x, y, \lambda)$ for $i_{1}<i$. Take a cutoff $\chi(y)$ which has support in $(-1,1)$ and equals 1 on $(-1 / 2,1 / 2)$. Let $a_{i} \in \mathcal{B}_{*}^{K-i+\beta, J}([-2,2])$ have support in $[-1,1] \times[0,1]$. With constants $\delta_{i}>0$ to be determined, consider

$$
g_{i}(x, y, \lambda)=\int_{\mathbf{R}} a_{i}(x-y z, \lambda) \phi(z) d z, \quad b_{i}(x, y, \lambda)=\frac{1}{i !} y^{i} g_{i}(x, y, \lambda) \chi\left(\delta_{i}^{-1} y\right) .
$$

It is clear that $\partial_{\lambda}^{j} g_{i}(\cdot, \lambda)$ are $\mathcal{C}^{\infty}$ away from $y=0$ and $g_{i} \in \mathcal{C}_{*}^{0, J}\left(\overline{V^{*}}\right)$. Also $g_{i}$ have support in $V^{*} \times[0,1]$.

To show that $b_{i} \in \mathcal{B}_{*}^{K+\beta, j}\left(\overline{V^{*}}\right)$, it suffices to show that $\partial_{\lambda}^{j} \partial^{I} b_{i}$ extend to functions in $\mathcal{B}_{*}^{\beta, 0}\left(\overline{V^{*}}\right)$ for all $I$ with $|I|=k<K+1$. We first derive a formula for derivatives. Write $I=I_{1}+I_{2}$ with $\left|I_{2}\right|=\min \{k, K-i\}$. We have

$$
\begin{aligned}
\partial^{I_{2}} \int a_{i}(x-y z, \lambda) \phi(z) d z & =\int \partial^{I_{2}}\left(a_{i}(x-y z, \lambda)\right) \phi(z) d z \\
& =\int\left(\partial^{\left|I_{2}\right|} a_{i}\right)(x-y z, \lambda) \phi_{I_{2}}^{(0)}(z) d z .
\end{aligned}
$$

Here and for the rest of the proof, $\phi_{*}^{(m)}(z)$ denotes a linear combination of $z^{l} \phi^{(n)}(z)$ with $l \leq k$ and $n \leq m$. Assume now that $y \neq 0$. Changing variables and interchanging the differentiation and integration, we get

$$
\partial^{I_{1}} \int\left(\partial^{\left|I_{2}\right|} a_{i}\right)(x-y z, \lambda) \phi_{I_{2}}(z) d z=\int \frac{1}{y^{\left|I_{1}\right|+1}}\left(\partial^{\left|I_{2}\right|} a_{i}\right)(z, \lambda) \phi_{I_{2} I_{1}}^{\left(\left|I_{1}\right|\right)}\left(\frac{x-z}{y}\right) d z .
$$

Changing variables again for the last integral, we get the formula

$$
\partial^{I_{1}+I_{2}} \int a_{i}(x-y z, \lambda) \phi(z) d z=y^{-\left|I_{1}\right|} \int\left(\partial^{\left|I_{2}\right|} a_{i}\right)(x-y z) \phi_{I_{1} I_{2}}^{\left(\left|I_{1}\right|\right)}(z) d z, \quad y \neq 0
$$

First consider the case where $i \leq k<K+1$. For $|I|=k$ and $y \neq 0$, we have

$$
\partial^{I}\left(y^{i} g_{i}(x, y, \lambda)\right)=\sum_{i_{1}+\left|I_{2}\right|=k} C_{i_{1} I_{2}} \partial_{y}^{i_{1}} y^{i} \partial^{I_{2}} \int a_{i}(x-y z, \lambda) \phi(z) d z
$$


with $i_{1} \leq i$. Write $I_{2}=I_{3}+I_{4}$ with $\left|I_{3}\right|=k-i$ and $\left|I_{4}\right|=i-i_{1}$. By (8.5) we get

$$
\partial^{I}\left(y^{i} g_{i}(x, y, \lambda)\right)=\sum_{i_{1} \leq i} \int\left(\partial^{k-i} a_{i}\right)(x-y z, \lambda) \phi_{I_{i i}}^{\left(i-i_{1}\right)}(z) d z, \quad|I|=k .
$$

It is obvious that the right-hand side extends to a function of class $\mathcal{B}_{*}^{\beta, j}\left(\overline{V^{*}}\right)$. Also, the $\mathcal{C}^{\beta}\left(\overline{V^{*}}\right)$ norm of $\partial_{\lambda}^{j} D^{I}\left(y^{i} g_{i}(x, y, \lambda)\right)$ in variables $x, y$ is bounded by $C_{k, i}\left|a_{i}\right|_{k-i+\beta, j}$. By dilation, it is easy to see that $\chi^{\delta_{i}}(z)=\chi\left(\delta_{i}^{-1} z\right)$ with $0<\delta_{i}<1$ satisfy $\left|\chi^{\delta_{i}}\right|_{k+\beta}<C_{k} \delta_{i}^{-k-\beta}$. Therefore,

$$
\left|b_{i}\right|_{k+\beta, j} \leq C_{k, i} \delta_{i}^{-k-\beta}\left|a_{i}\right|_{k-i+\beta, j}, \quad 0 \leq i \leq k .
$$

Next, we want to verify that $\partial_{y}^{i}\left(y^{i} g_{i}(x, y, \lambda)\right)=i ! a_{i}(x, \lambda)$ at $y=0$. Fix $x$. By $a_{i} \in \mathcal{C}_{*}^{0, j}([-2,2]), \operatorname{supp} a_{i} \subset[-1,1] \times[0,1]$ and (8.6) with $k=i$, the value of $\partial_{y}^{i}\left(y^{i} g_{i}(x, y, \lambda)\right)$ at $y=0$ depends only on $a_{i}(x)$. However, the identity holds trivially for any $\delta_{i} \in(0,1)$, when $a_{i}$ is constant. We now determine $a_{i}$ by taking $a_{0}=f_{0}$, and

$$
a_{i}=f_{i}-\left.\partial_{y}^{i}\right|_{y=0}\left(b_{0}(x, y, \lambda)+\cdots+b_{i-1}(x, y, \lambda)\right) .
$$

By (8.7), for $j<J+1$ and $i+k<K+1$ we get

$$
\begin{aligned}
\left|a_{i}\right|_{k+\beta, j} & \leq\left|f_{i}\right|_{k+\beta, j}+\sum_{l<i} C_{i+k} \delta_{i-1}^{-i-k-\beta}\left|a_{l}\right|_{k+i-l+\beta, j} \\
& \leq\left|f_{i}\right|_{k+\beta, j}+\delta_{i-1}^{-(i+k+\beta) l} \sum_{l<i} C_{i+k}^{\prime}\left|f_{l}\right|_{k+i-l+\beta, j} .
\end{aligned}
$$

Here we have assumed that $\delta_{l}$ decreases. In particular, $a_{i}$ is in $\mathcal{B}_{*}^{K-i+\beta, J}([-2,2])$. We have achieved

$$
\partial_{y}^{i}\left(b_{0}(x, y, \lambda)+\cdots+b_{i}(x, y, \lambda)\right)=f_{i}(x, \lambda), \quad y=0 .
$$

Now consider the case where $i>k=|I|$. By the product rule and (8.5),

$$
\begin{gathered}
\partial^{I}\left(y^{i} g_{i}(x, y, \lambda) \chi\left(\delta_{i}^{-1} y\right)\right)=\sum C_{i_{1} i_{2} I_{3}} \partial_{y}^{i_{1}} y^{i} \cdot \partial_{y}^{i_{2}} \chi\left(\delta_{i}^{-1} y\right) \cdot \partial^{I_{3}} g_{i}(x, y, \lambda) \\
=\sum \tilde{C}_{i_{1} i_{2} I_{3}} \partial_{y}^{i_{2}} \chi\left(\delta_{i}^{-1} y\right) \cdot y^{i-i_{1}-\left|I_{3}\right|} \int a_{i}(x-y z, \lambda) \phi^{\left(\left|I_{3}\right|\right)}(z) d z
\end{gathered}
$$

Here the summation runs over $i_{1}+i_{2}+\left|I_{3}\right|=k$. The $\mathcal{C}^{\beta}$ norm of $\left(\delta_{i}^{-1} y\right)^{i-i_{1}-\left|I_{3}\right|}$. $\chi^{\left(i_{2}\right)}\left(\delta_{i}^{-1} y\right)$ is bounded by $C \delta_{i}^{-\beta}$. Thus for any $\delta_{i} \in(0,1)$,

$$
\begin{gathered}
\partial_{y}^{k}\left(b_{i}(x, y, \lambda)\right)=0, \quad y=0, \quad k<i, \\
\left|b_{i}\right|_{k+\beta, j} \leq C_{k, i} \delta_{i}^{i-k-\beta}\left|a_{i}\right|_{\beta, j}, \quad k<i .
\end{gathered}
$$

By (8.13) we inductively choose decreasing $\delta_{i}$ such that

$$
\left|b_{i}\right|_{i-1+\beta, j_{i-1}} \leq \delta_{i}^{1-\beta}\left|a_{i}\right|_{\beta, j_{i-1}} \max _{k<i} C_{k, i}<\frac{\epsilon_{i}}{2^{i}}, \quad i \geq 1 .
$$

Take $E f(x, y, \lambda)=\sum_{i+J \leq K} b_{i}(x, y, \lambda)$. By (8.14), we get $\sum_{i>k}\left|b_{i}\right|_{k+\beta, j_{k}}<\epsilon_{k+1}$ for $0 \leq k<K$. Combining it with (8.10), (8.12) and (8.7), we obtain $\partial_{y}^{i} E f=f_{i}$ at $y=0$ and (8.1), respectively. Combining (8.7) for $k=K$ and (8.9) gives us (8.2). From (8.4) and (8.6), we see that $b_{k} \in \mathcal{C}_{*}^{K+\beta, J}\left(\overline{V^{*}}\right)$ when $f_{k} \in \mathcal{C}_{*}^{K-k+\beta, J}\left(\overline{V^{*}}\right)$. Using the convergence of $\sum\left|b_{i}\right|_{k+\beta, j_{k}}$ again, we obtain $E f \in \mathcal{C}_{*}^{K+\beta, J}\left(\overline{V^{*}}\right)$. The dependence of $E f$ and $C_{k}(\epsilon, f)$ on norms of $f_{i}$, as stated in the lemma, is determined by (8.3), (8.9) with $k=0$ and (8.14). 
Note that (ii) immediately follows from the extension formulae. Indeed, the partition of unity for $\partial \Omega$ preserves conditions $\partial_{\nu}^{i} f=0$ for $i>0$ and $f_{0}$ being constant in a neighborhood of $z_{0}$ in $\partial \Omega$, by starting with one of the $\chi_{p}$ 's to be 1 near $z_{0}$ and all other $\chi_{p}$ 's to be 0 near $z_{0}$. From (8.3), we have $\partial_{y}^{i} f=0$ for $i>0$ near $z_{0}$. By (8.4) and shrinking the support of $\phi$ if necessary, $E f$ is constant near $z_{0}$.

Lemma 8.2. Let $J, K, \beta, \epsilon_{k+1}, j_{k}$ be as in Lemma 8.1. Assume further that $J \leq K$, and $K>k+j_{k}$ for $0<k<K$. Let $\Omega$ be a bounded domain with $\partial \Omega \in \mathcal{C}^{K+\beta} \cap \mathcal{C}^{1}$. Suppose that $f_{i} \in \mathcal{B}_{*}^{K-i+\beta, J}(\partial \Omega)$ (resp. $\mathcal{C}_{*}^{K-i+\beta, J}(\partial \Omega)$ ) for all $i \geq 0$ satisfying $i+J \leq K$. There exists $E f \in \mathcal{B}^{K+\beta, J}(\bar{\Omega})\left(\right.$ resp. $\left.\mathcal{C}^{K+\beta, J}(\bar{\Omega})\right)$ satisfying $\partial_{\nu}^{i} E f=f_{i}$ for $i+J \leq K$. Furthermore,

$$
\begin{aligned}
& \|E f\|_{k+\beta, j_{k}} \leq \epsilon_{k+1}+C_{k}(\epsilon, f) \sum_{i \leq k, i+J \leq K}\left\|f_{i}\right\|_{k-i+\beta, j_{k}}, \quad k<K \leq \infty, \\
& \|E f\|_{K+\beta, j} \leq C_{K}(\epsilon, f) \sum_{i \leq K-J}\left\|f_{i}\right\|_{K-i+\beta, j}, \quad K<\infty, \quad 0 \leq j<J+1,
\end{aligned}
$$

where the extension operator $f \rightarrow E f$ depends only on $i, \partial \Omega$, and the upper bound $M_{i}$ of $\epsilon_{i}^{-1}$ and $\left\|f_{l}\right\|_{i-l+j_{i-1}+\beta, j_{i-1}}$ for $l \leq i, i>0$ and $i+J \leq K$. Furthermore, $C_{k}(\epsilon, f)$ depends on $k, \partial \Omega$ and $M_{l}$ for $l \leq k ;$ Ef is constant near $\left(z_{0}, 0\right) \in \bar{\Omega} \times[0,1]$ if near $z_{0} \in \partial \Omega, f_{0}-f_{0}\left(z_{0}\right)$ and $f_{i}$ vanish for $i>0$ and $i+J \leq K$.

Proof. We use $E f=\sum_{i+J \leq K} b_{i}$, with $b_{i}$ being of the form (8.4). We still have $\partial_{y}^{k} b_{i}=0$ for $k<i$ and $\partial_{y}^{i} b_{i}=a_{i}$ at $\lambda=0$ as they hold for $\delta_{i} \in(0,1)$, provided $a_{i} \in \mathcal{B}^{K-i+\beta, J}(\partial \Omega)\left(\subset \mathcal{B}_{*}^{K-J-i, J}(\partial \Omega)\right)$. We rewrite previous estimates in norms $\|\cdot\|$ instead of $|\cdot|$. Assume that $i+J \leq K, j<J+1$, and $(j \leq) k<K+1$. By (8.7) and (8.13), we have

$$
\begin{aligned}
\left\|b_{i}\right\|_{k+\beta, j}= & \max _{l \leq j}\left|b_{i}\right|_{k-l+\beta, l} \leq \max _{l \leq j}\left\{C_{k, i} \delta_{i}^{-k-\beta}\left|a_{i}\right|_{k_{1}+\beta, l}\right\} \\
& \leq C_{k, i} \delta_{i}^{-k-\beta}\left\|a_{i}\right\|_{k_{2}+\beta, j}, \quad i \leq k .
\end{aligned}
$$

Here $k_{1}=\max \{k-l-i, 0\}$ and $k_{2}=\max \{k-i, j\} \leq K-i$. By (8.13) again, we have

$$
\left\|b_{i}\right\|_{k+\beta, j}=\max _{l \leq j}\left|b_{i}\right|_{k-l+\beta, l} \leq C_{k} \delta_{i}^{1-\beta}\left\|a_{i}\right\|_{j+\beta, j}, \quad i>k .
$$

Assume further that $l \leq i$. By (8.17) we get

$$
\begin{aligned}
\left\|a_{l}\right\|_{k+\beta, j} & \leq\left\|f_{l}\right\|_{k+\beta, j}+\sum_{m<l}\left\|b_{m}\right\|_{k+l+\beta, j} \\
& \leq\left\|f_{l}\right\|_{k+\beta, j}+\delta_{i-1}^{-(i+k+\beta)} \sum_{m<l} C_{i}\left\|a_{m}\right\|_{k+l-m+\beta, j}, \\
\left\|a_{l}\right\|_{k+\beta, j} & \leq\left\|f_{l}\right\|_{k+\beta, j}+\delta_{i-1}^{-(i+k+\beta) i} \sum_{m<l} C_{i}^{\prime}\left\|f_{m}\right\|_{k+l-m+\beta, j} .
\end{aligned}
$$

Thus, by (8.19) with $l=i, a_{i}$ is in $\mathcal{B}^{K-i+\beta, J}$; by 8.17)-8.18), $b_{i} \in \mathcal{B}^{K+\beta, J}$. Therefore, by (8.18)-(8.19), we can inductively choose decreasing $\delta_{i}$ such that

$$
\left\|b_{i}\right\|_{i-1+\beta, j_{i-1}} \leq C_{i} \delta_{i}^{1-\beta}\left\|a_{i}\right\|_{j_{i-1}+\beta, j_{i-1}}<\frac{\epsilon_{i}}{2^{i}}
$$


where $\delta_{i}$ depends on the upper bound of $C_{i}>1, \epsilon_{i}^{-1}$ and $\left\|f_{l}\right\|_{i-l+j_{i-1}+\beta, j_{i-1}}$ for $l \leq i$. The rest of the arguments in the previous proof are valid.

The above proof for the non-parameter case without estimate on norms is in [5] (pp. 16 and 18). See also 2] for different spaces with parameter. For the proof of Theorem 1.2, we need the following extension lemma to prescribe $\lambda$-derivatives.

Lemma 8.3. Let $J, K$ be integers or $\infty$ and let $0 \leq \beta<1$. Let $\Omega$ be a bounded domain with $\partial \Omega \in \mathcal{C}^{K+\beta} \cap \mathcal{C}^{1}$.

(i) Suppose that $f_{j} \in \mathcal{C}^{K+\beta}(\partial \Omega)$ for $0 \leq j<J+1$. There exists $E f \in$ $\mathcal{C}_{*}^{K+\beta, J}(\partial \Omega)$ satisfying $\partial_{\lambda}^{j} E f=f_{j}$ for $0 \leq j<J+1$ at $\lambda=0$.

(ii) Let $J \leq K$. Suppose that $f_{j} \in \mathcal{C}^{K-j+\beta}(\partial \Omega)$ for $0 \leq j<J+1$. There exists $E f \in \mathcal{C}^{K+\beta}(\partial \Omega \times[0,1])$ satisfying $\partial_{\lambda}^{j} E f=f_{j}$ for $0 \leq j<J+1$ at $\lambda=0$; in particular, $E f \in \mathcal{B}^{K+\beta, K}(\partial \Omega)$.

(iii) In $(i)$ and (ii), if near $p \in \partial \Omega, f_{0}$ is constant and $f_{i}$ vanish for $i>0$, then $E f$ is constant on $V \times[0,1]$ for some neighborhood $V$ of $p$.

(iv) (i), (ii) and (iii) hold if $\bar{\Omega}$ substitutes for $\partial \Omega$.

Proof. (i) When $J$ is finite, we simply take $E f(x, \lambda)=\sum_{j=0}^{J} \lambda^{j} f_{j}(x)$. Assume that $J=\infty$. Let $\chi(\lambda)$ be a $\mathcal{C}^{\infty}$ function which has support in $[0,1 / 2]$ and equals 1 near $\lambda=0$. We choose $0<\delta_{j}<1 / 2$ satisfying $\delta_{j}\left|f_{j}\right|_{2 j}|\chi|_{j}<2^{-j}$. Then $\operatorname{Ef}(x, \lambda)=$ $\sum \lambda^{j} f_{j}(x) \chi\left(\delta_{j}^{-1} \lambda\right)$ is a desired extension.

(ii)-(iii) The extension $E f$ is a special case of Lemma 8.1 where the parameter $\lambda$ is absent and the variable $y$ in its proof is replaced by $\lambda$. We first find an extension $E f \in \mathcal{B}^{K+\beta, J}(\partial \Omega)$. Using a partition of unity and local change of coordinates of class $\mathcal{C}^{K+\beta} \cap \mathcal{C}^{1}$, we may assume that $\partial \Omega$ contains $[-2,2] \times\{0\}, \bar{\Omega}$ contains $[-2,2] \times[0,1]$, and $f_{i}$ have support in $[-1 / 4,1 / 4] \times\{0\}$. Locally we find an extension $E f \in \mathcal{C}^{K+\beta}([-2,2] \times[0,1])$ such that $\partial_{y}^{j} E f(x, 0)=f_{j}(x)$ and $\operatorname{supp} E f \subset[-1,1] \times$ $[0,1 / 2]$. Then $\operatorname{Ef}(x, \lambda)$ is a desired extension. It is clear that (iii) follows from the extension formulae.

(iv) For the extension $E f \in \mathcal{C}^{K+\beta}(\bar{\Omega} \times[0,1])$, again by partition of unity for $\bar{\Omega}$, we may assume that all $f_{i}$ have support in $(-1 / 4,1 / 4) \times[0,1 / 4)$. Next, we apply Lemma 8.1 for the non-parameter version and extend $f_{i}$ across the boundary of $\partial \Omega$ to $(-1 / 2,1 / 2) \times(-1 / 2,1 / 2)$. We still have $f_{i} \in \mathcal{C}^{K-i+\beta}$ and $f_{i}$ has compact support. We substitute (8.4) with

$$
g_{i}(x, \lambda)=\int_{\mathbf{R}^{2}} a_{i}(x-\lambda z) \phi(z) d z, \quad b_{i}(x, \lambda)=\frac{1}{i !} \lambda^{i} g_{i}(x, \lambda) \chi\left(\delta_{i}^{-1} \lambda\right),
$$

where $a_{i} \in \mathcal{C}^{K+\beta-i}\left([-3 / 4,3 / 4]^{2}\right)$ and $\operatorname{supp} a_{i} \subset(-1 / 2,1 / 2)^{2}$. The arguments in the proof of Lemma 8.1 are written for one variable $x$. However, when $x \in$ $\mathbf{R}^{2}$ or in higher dimensional Euclidean spaces, the identities require only minor changes. We will leave the details to the reader. In conclusion, one can find $E f(x, \lambda)=\sum b_{i}(x, \lambda)$ such that $E f \in \mathcal{C}^{K+\beta}\left([-2,2]^{2} \times[0,1]\right) \subset \mathcal{B}^{K+\beta, K}\left([-2,2]^{2}\right)$, $\operatorname{supp} E f \subset[-1,1]^{2} \times[0,1]$ and $\partial_{\lambda}^{j} E f=f_{j}$.

Remark 8.4. As shown in Lemma 2.2, the composition of functions is restrictive for spaces $\mathcal{C}^{k+\alpha, j}$. We do not know if the $g_{i}$ in (8.20) are of class $\mathcal{C}^{K+\alpha, j}(\bar{\Omega})$ when $K+\alpha$ is finite but not an integer; therefore, we do not know if there exists an extension $E f$ in (ii) of Lemma 8.3 that is of class $\mathcal{C}^{K+\alpha, J}(\bar{\Omega})$. 
Lemma 8.5. Let $1 \leq k \leq \infty$ and $0 \leq \beta<1$. Let $\Omega_{i}$ be bounded domains of the $\mathcal{C}^{k+\beta}$ boundary. Let $\gamma$ be an orientation preserving $\mathcal{C}^{k+\beta}$ diffeomorphism from $\partial \Omega_{1}$ onto $\partial \Omega_{2}$, which sends outer boundary to outer boundary. Then $\gamma$ extends to a $\mathcal{C}^{k+\beta}$ diffeomorphism from $\bar{\Omega}_{1}$ onto $\bar{\Omega}_{2}$ and it also extends to a $\mathcal{C}^{k+\beta}$ diffeomorphism from $\overline{\Omega_{1}^{\prime}}$ onto $\overline{\Omega_{2}^{\prime}}$ which is the identity on $|z|>R$ when $R$ is sufficiently large.

Proof. We first prove the assertions when $\Omega_{i}$ are simply connected. Let $\gamma_{1}: \partial \mathbb{D} \rightarrow$ $\partial \Omega_{1}$ be a $\mathcal{C}^{k+\beta}$ parameterization. Approximate $\gamma_{1}$ in $\mathcal{C}^{1}$ norm by a $\mathcal{C}^{\infty}$ parameterization $\tilde{\gamma}_{1}: \partial \mathbb{D} \rightarrow \partial \tilde{\Omega}_{1}$. Then $\tilde{\gamma}_{1} \gamma_{1}^{-1}-I$ has a small $\mathcal{C}^{1}$ norm on $\partial \Omega_{1}$. By Whitney's extension theorem, it extends to a $\mathcal{C}^{k+\beta}$ mapping $\varphi$ which maps from $\overline{\Omega_{1}}$ into $\mathbf{C}$ with a small $\mathcal{C}^{1}$ norm. Then $I+\varphi$ is a $\mathcal{C}^{k+\beta}$ diffeomorphism mapping $\overline{\Omega_{1}}$ onto $\overline{\Omega_{1}^{*}}$ with $\mathcal{C}^{\infty}$ boundary. Therefore, we may assume that $\partial \Omega_{i}$ have $\mathcal{C}^{\infty}$ boundary. Thus, we may further assume that $\Omega_{i}$ are the unit disc, say, by Kellogg's Riemann mapping theorem. Since $\gamma$ preserves the orientation of the unit circle, then $\gamma\left(e^{i \theta}\right)=e^{i(\theta+a(\theta))}$. Here $a$ is $2 \pi$-periodic and $1+a^{\prime}>0$. Let $\rho:[0, \infty) \rightarrow[0,1]$ be a smooth function which has support in $(1 / 2,2)$ and equals 1 near 1 . Then $\Gamma_{0}\left(r e^{i \theta}\right)=r e^{i(\theta+\rho(r) a(\theta))}$ is a desired extension.

To extend $\gamma$ to the unbounded component, using time-one mappings of vector fields of compact support, we may assume that $0 \in \Omega_{i}$. Using the inversion $\iota_{0}(z)=1 / z$ it suffices to show that in the above arguments we can extend $\gamma$ to a $\mathcal{C}^{k+\beta}$ diffeomorphism from $\overline{\Omega_{1}}$ onto $\overline{\Omega_{2}}$, which is the identity map near the origin. Composing $\Gamma_{0}$ with the time-one map of a vector field which vanishes near $\partial \Omega_{1}$, we may assume that $\Gamma_{0}(0)=0$. Using a dilation, we may assume that $\Gamma_{0}(z)=\hat{\Gamma}_{0}(z)+E(z)$, where $|E|+|\partial E|<\epsilon$ on $|z|<1 / 2$ and $\hat{\Gamma}_{0}$ is the linear part of $\Gamma_{0}$ at $z=0$. Let $\chi=0$ on $|z|<1 / 4$ and $\chi=1$ on $|z|>1 / 2$. When $\epsilon$ is small, $\Gamma_{1}(z)=\Gamma_{0}(z)+\chi(|z|) E(z)$ is still a $\mathcal{C}^{k+\beta}$ diffeomorphism. Now $\Gamma_{1}$ is linear near 0 . Since $\Gamma_{1}^{\prime}(0)$ preserves orientation, by the Jordan normal form of $2 \times 2$ matrices we find two flows $X^{t}$ and $Y^{t}$ of vector fields vanishing at 0 such that $\Gamma_{1}^{\prime}(0)=X^{1} \circ Y^{1}$. Let $\rho$ be a cutoff function which equals 1 near the origin and has support in a small neighborhood of the origin. Then $(\rho Y)^{-1} \circ(\rho X)^{-1} \circ \Gamma_{1}$ is a desired extension.

The general case for bounded domains is obtained by induction on $m+1$, the number of components of $\partial \Omega_{i}$. We have proved the lemma when $m=0$. Let $C_{1}$ be a component of the inner boundary of $\Omega_{1}$ and let $C_{2}=\gamma\left(C_{1}\right)$. Let $\omega_{i}$ be bounded components of $\mathbf{C} \backslash C_{i}$. Applying results proved in the previous paragraph, we find an extension $\Gamma_{1}$ of $\left.\gamma\right|_{\partial \omega_{1}}$ to $\overline{\omega_{1}^{\prime}}$. Replacing $\gamma$ by $\Gamma_{1} \circ \gamma$, we may assume that $\gamma$ is the identity on $C_{1}$. Using a diffeomorphism of class $\mathcal{C}^{k+\beta}$ from $\overline{\omega_{1}^{\prime}}$ onto $\mathbf{C} \backslash \mathbb{D}$, we may assume that $C_{1}=C_{2}$ is the unit circle. Let $\tilde{\Omega}_{i}=\partial \Omega_{i} \cup \overline{\mathbb{D}}$. We know that $\gamma$ extends to a $\mathcal{C}^{k+\beta}$ diffeomorphism $\Gamma_{0}$ from $\overline{\tilde{\Omega}_{1}}$ onto $\overline{\tilde{\Omega}_{2}}$. By the argument in the previous paragraph, we may achieve $\Gamma_{0}$ to be the identity on $|z|<\epsilon$ for some $0<\epsilon<1$. Let $\Gamma_{2}$ be a $\mathcal{C}^{\infty}$ diffeomorphism on $\mathbf{C}$ which is the identity on the complement of the disc $\mathbb{D}_{\rho}$ and sends $\overline{\mathbb{D}}$ into $\mathbb{D}_{\epsilon}$. Here $\rho>1$ and $\bar{D}_{\rho}$ is contained in $\tilde{\Omega}_{1}$. Then $\Gamma_{2}^{-1} \circ \Gamma_{0} \circ \Gamma_{2}$ is a desired extension of $\gamma$ to $\overline{\Omega_{1}}$.

The proof of the next lemma needs Theorem 9.2 for the Dirichlet problem for interior domains. Our arguments are valid because Theorem 9.2 is for embeddings $\gamma^{\lambda}$ which are restrictions of $\Gamma^{\lambda}$.

Lemma 8.6. Let $j, k$ be non-negative integers or $\infty$. Let $0 \leq \beta<1$ and let $\Omega$ be a bounded domain in $\mathbf{C}$ with $\partial \Omega \in \mathcal{C}^{k+\beta} \cap \mathcal{C}^{1}$. Let $\gamma^{\lambda}$ be a family of orientationpreserving embeddings from $\partial \Omega$ onto $\partial \Omega^{\lambda}$ with $\gamma \in \mathcal{C}^{1,0}(\partial \Omega)$. Assume that $\gamma^{\lambda}$ sends 
outer boundary to outer boundary. For each $\lambda_{0} \in[0,1]$, there exists $\delta>0$ such that if $I=[0,1] \cap\left[\lambda_{0}-\delta, \lambda_{0}+\delta\right]$ substitutes for $[0,1]$ in all function spaces, then $\gamma^{\lambda}$ extend to $\mathcal{C}^{1}$ embeddings $\Gamma^{\lambda}$ from $\bar{\Omega}$ onto $\overline{\Omega^{\lambda}}$ with $\Gamma \in \mathcal{C}^{1,0}(\bar{\Omega})$. Furthermore, if $\gamma$ is in $\mathcal{B}_{*}^{k+\beta, j}(\partial \Omega), \mathcal{C}_{*}^{k+\beta, j}(\partial \Omega), \mathcal{B}^{k+\beta, j}(\partial \Omega)(k \geq j)$ and $\mathcal{C}^{k+\beta, j}(\partial \Omega)(k \geq j)$, there exists an extension $\Gamma$ in $\mathcal{B}_{*}^{k+\beta, j}(\bar{\Omega}), \mathcal{C}_{*}^{k+\beta, j}(\bar{\Omega}), \mathcal{B}^{k+\beta, j}(\bar{\Omega})$ and $\mathcal{C}^{k+\beta, j}(\bar{\Omega})$, respectively, and if $\partial \Omega$ and $\gamma$ are real analytic, then $\Gamma \in \mathcal{C}^{\omega}(\bar{\Omega} \times I)$.

Proof. With $\delta$ to be determined, set $I=\left[\lambda_{0}-\delta, \lambda_{0}+\delta\right] \cap[0,1]$. As stated in the lemma, the space $\mathcal{C}^{1,0}(\bar{\Omega})$ and others depend on $\delta$.

(i) We apply Lemma 8.5 and extend $\gamma^{\lambda_{0}}$ to a $\mathcal{C}^{1}$ diffeomorphism $\Gamma_{0}^{\lambda_{0}}$ from $\bar{\Omega}$ onto $\overline{\Omega^{\lambda_{0}}}$. Approximate $\Gamma_{0}^{\lambda_{0}}$ by a smooth map $\Gamma_{1}^{\lambda_{0}}$ and set $\Gamma_{1}^{\lambda}=\Gamma_{1}^{\lambda_{0}}$ for all $\lambda$. We have $\left|\gamma^{\lambda}-\Gamma_{1}^{\lambda}\right|_{1}<\epsilon<\epsilon_{0}$ for $\lambda \in I$ when $\delta$ is sufficiently small. We apply Lemma 8.1 and extend $\gamma-\Gamma_{1}$ to an element $\Gamma_{2} \in \mathcal{B}_{*}^{k+\beta, j}(\bar{\Omega}) \cap \mathcal{C}^{1,0}(\bar{\Omega})$ such that $\left|\Gamma_{2}\right|_{1,0}<\epsilon_{0}+C\left(\epsilon_{0}\right) \epsilon<2 \epsilon_{0}$. Then $\Gamma^{\lambda}=\Gamma_{2}^{\lambda}+\Gamma_{1}^{\lambda}$ are extensions of $\gamma^{\lambda}$. Also, $\left|\Gamma^{\lambda_{0}}-\Gamma_{0}^{\lambda_{0}}\right|_{1} \leq 2 \epsilon_{0}$. Since $\Gamma_{0}^{\lambda_{0}}$ is an embedding, then $\Gamma^{\lambda_{0}}$ is also an embedding when $\epsilon_{0}$ is sufficiently small. By continuity in the $\mathcal{C}^{1}$ norm, we know that $\Gamma^{\lambda}$ are embeddings for $\lambda \in I$ when $\delta$ is sufficiently small. Analogously, we can find the extensions for the other three cases.

(ii) For the real analytic case, the proof in (i) via extension does not apply. Instead, we solve a Dirichlet problem with parameter. We extend $\gamma^{\lambda_{0}}$ to a smooth embedding $\Gamma^{0}$ and approximate $\Gamma^{0}$ by real analytic embeddings $\Gamma^{1 / j}$ such that $\left|\Gamma^{1 / j}-\Gamma^{0}\right|_{3 / 2}<1 / j$. For $f \in \mathcal{C}^{3 / 2}\left(\partial \Omega^{1 / j}\right)$, let $T_{j} f$ be the unique harmonic function $\Omega_{j}$ which is continuous up to the boundary and has boundary value $f$. Thus $T_{j}$ maps $\mathcal{C}^{3 / 2}\left(\partial \Omega^{1 / j}\right)$ into $\mathcal{C}^{3 / 2}\left(\overline{\Omega^{1 / j}}\right)$. We know that $T_{j}$ is injective and the range of $T_{j}$ is the Banach space of harmonic functions on $\Omega_{j}$ of class $\mathcal{C}^{3 / 2}\left(\overline{\Omega^{1 / j}}\right)$. The inverse mapping of $T_{j}$ is the restriction mapping, which is obviously bounded. By the open mapping theorem, $T_{j}$ is bounded with norm $\left\|T_{j}\right\|$. Next, we want to show that the norms $\left\|T_{j}\right\|$ are bounded too. Define

$$
\Gamma^{\theta /(j+1)+(1-\theta) / j}=\theta \Gamma^{1 /(j+1)}+(1-\theta) \Gamma^{1 / j}, \quad 0 \leq \theta \leq 1 .
$$

Then $\left\{\Gamma^{\lambda}\right\} \in \mathcal{C}^{3 / 2,0}(\bar{\Omega})$. When $\lambda$ is sufficiently small, $\Gamma^{\lambda}$ embeds $\bar{\Omega}$ onto $\overline{\Omega^{\lambda}}$. Assume for the sake of contradiction that $\left\|T_{j}\right\|$ are not bounded. We find $f^{1 / j} \in \mathcal{C}^{3 / 2}\left(\partial \Omega^{1 / j}\right)$ such that $\left|T_{j} f^{1 / j}\right|_{3 / 2}=1$ and $\left|f^{1 / j}\right|_{3 / 2} \rightarrow 0$ as $j \rightarrow \infty$. Define

$$
f^{\theta /(j+1)+(1-\theta) / j} \circ \Gamma^{\theta /(j+1)+(1-\theta) / j}=\theta f^{1 /(j+1)} \circ \Gamma^{1 /(j+1)}+(1-\theta) f^{1 / j} \circ \Gamma^{1 / j} .
$$

Then $f \in \mathcal{C}^{3 / 2,0}\left(\partial \Omega_{\Gamma}\right)$ for $f^{0}=0$. Let $u^{\lambda}$ be the harmonic function on $\Omega^{\lambda}$ which is continuous up to boundary and has boundary value $f^{\lambda}$. Thus $u \in \mathcal{C}^{3 / 2,0}\left(\bar{\Omega}_{\Gamma}\right)$ and $u^{0}=0$ because $f^{0}=0$. However, $\left|u^{1 / j}\right|_{3 / 2}=1$, a contradiction.

Let $u_{\lambda}^{1 / j}$ be harmonic on $\Omega^{1 / j}$ such that $v_{j}^{\lambda}=u_{\lambda}^{1 / j} \circ \Gamma^{1 / j}(z)=\gamma^{\lambda}(z)-\Gamma^{1 / j}(z)$. We have $\left|u_{\lambda}^{1 / j}\right|_{3 / 2} \leq\left\|T_{j}\right\| \cdot\left|\gamma^{\lambda}-\Gamma^{1 / j}\right|_{3 / 2} \rightarrow 0$ as $j \rightarrow \infty$ and $\lambda \rightarrow 0$. Hence $v_{j}^{\lambda}+\Gamma^{1 / j}$ approach to $\Gamma^{0}$ in $\mathcal{C}^{3 / 2}$ norms as $\lambda$ and $1 / j$ tend to zero. Fix a $j$ such that $v_{j}^{\lambda}+\Gamma^{1 / j}$ are embeddings for all $\left|\lambda-\lambda_{0}\right|$ sufficiently small. Then $\Gamma_{0}^{\lambda}=v_{j}^{\lambda}+\Gamma^{1 / j}$ are extensions of $\gamma^{\lambda}$. Finally, $\Gamma_{0}^{\lambda}(z)$ is a real analytic function on $\bar{\Omega} \times I$ by the analyticity of solutions of the Dirichlet problem with parameter.

We now introduce spaces for exterior domains. Let $\Omega^{\prime}=\mathbf{C} \backslash \bar{\Omega}$. Without loss of generality, we assume that $\Omega$ is bounded and simply connected. Motivated by 
the definition that a function $h(z)$ is harmonic at $\infty$ if $h(1 / z)$ is harmonic at the origin, we define inversions

$$
\iota_{a}(z)=\frac{1}{z-a}+a, \quad \Omega_{a}=\{a\} \cup \iota_{a} \Omega^{\prime}, \quad \Omega_{b}^{\lambda}=\left\{b_{\lambda}\right\} \cup \iota_{b_{\lambda}}\left(\Omega^{\lambda}\right)^{\prime}
$$

for $a \in \Omega$ and $b_{\lambda} \in \Omega^{\lambda}$. For a family of embeddings $\Gamma^{\lambda}$ from $\overline{\Omega^{\prime}}$ onto $\overline{\left(\Omega^{\lambda}\right)^{\prime}}$, define

$$
\Gamma_{b}^{\lambda}=\iota_{b_{\lambda}} \circ \Gamma^{\lambda}, \quad \Gamma_{a, b}^{\lambda}=\iota_{b_{\lambda}} \circ \Gamma^{\lambda} \circ \iota_{a}, \quad \gamma_{b}^{\lambda}=\iota_{b_{\lambda}} \circ \gamma^{\lambda}, \quad \gamma_{a, b}^{\lambda}=\iota_{b_{\lambda}} \circ \gamma^{\lambda} \circ \iota_{a} .
$$

Set $\Gamma_{a, b}^{\lambda}(a)=b_{\lambda}$. Then $\Gamma_{a, b}^{\lambda}$ is a fractional linear map from $\Omega_{a}$ onto $\Omega_{b}^{\lambda}$.

We denote $f \in \mathcal{C}^{k+\alpha}\left(\overline{\Omega^{\prime}}\right)$ if $f \circ \iota_{a}$, which is not defined at $a$, extends to an element in $\mathcal{C}^{k+\alpha}\left(\overline{\Omega_{a}}\right)$. Denote $f=\left\{f^{\lambda}\right\} \in \mathcal{C}^{k+\alpha, j}\left(\overline{\Omega^{\prime}}\right)$ (resp. $\mathcal{B}^{k+\beta, j}\left(\overline{\Omega^{\prime}}\right)$ ) if $f \circ \iota_{a}$ extends to an element in $\mathcal{C}^{k+\alpha, j}\left(\overline{\Omega_{a}}\right)$ (resp. $\mathcal{B}^{k+\beta, j}\left(\overline{\Omega_{a}}\right)$ ). We emphasize that as in (8.21) $-(8.22)$ we require $a \in \Omega$. The extended functions are still denoted by $f \circ \iota_{a}$. It is easy to verify that the definitions are independent of the choices of $a$. Let $\Gamma^{\lambda}$ be a family of $\mathcal{C}^{1}$ embeddings from $\overline{\Omega^{\prime}}$ onto $\overline{\left(\Omega^{\lambda}\right)^{\prime}}$. Denote $f \in \mathcal{C}^{k+\alpha, j}\left(\overline{\Omega_{\Gamma}^{\prime}}\right)\left(\right.$ resp. $\left.\mathcal{B}^{k+\alpha, j}\left(\overline{\Omega_{\Gamma}^{\prime}}\right)\right)$ if $\left\{f^{\lambda} \circ \Gamma^{\lambda}\right\} \in \mathcal{C}^{k+\alpha, j}\left(\overline{\Omega^{\prime}}\right)$ (resp. $\mathcal{B}^{k+\beta, j}\left(\overline{\Omega^{\prime}}\right)$ ). The spaces for functions on boundaries of exterior domains will be the same as those for boundaries of interior domains.

To use the spaces $\mathcal{C}^{k+\alpha, j}\left(\overline{\Omega_{\Gamma}^{\prime}}\right)$ and $\mathcal{B}^{k+\alpha, j}\left(\overline{\Omega_{\Gamma}^{\prime}}\right)$, we will need good control of embeddings $\Gamma^{\lambda}$ at infinity. Suppose that $b_{\lambda}$ and $d_{\lambda}$ are in $\Omega^{\lambda}$ and $a, c$ are in $\Omega$. It is obvious that $\Gamma_{a, b}^{\lambda}=\iota_{b_{\lambda}} \circ \Gamma^{\lambda} \circ \iota_{a}$ extends to a $\mathcal{C}^{1}$ embedding from $\overline{\Omega_{a}}$ onto $\overline{\Omega_{b}^{\lambda}}$ if and only if $\Gamma_{c, d}^{\lambda}$ extends to a $\mathcal{C}^{1}$ embedding from $\overline{\Omega^{\prime}}$ onto $\overline{\Omega_{d}^{\lambda}}$ for any $c \in \Omega$ and $d_{\lambda} \in \Omega^{\lambda}$. By $\left\{b_{\lambda}\right\} \in \mathcal{C}^{j}([0,1])$, we mean that $\lambda \rightarrow b_{\lambda}$ is of class $\mathcal{C}^{j}([0,1])$. Then, $\Gamma_{a, b} \in \mathcal{C}^{k+\alpha, j}\left(\overline{\Omega_{a}}\right)$ if and only if $\Gamma_{c, d} \in \mathcal{C}^{k+\alpha, j}\left(\overline{\Omega_{d}}\right)$, provided $b$ and $d$ are in $\mathcal{C}^{j}([0,1])$.

To put the above definitions into context, we restate Lemma 2.2 (iii) as follows: The space $\mathcal{B}^{k+\beta, j}\left(\overline{\Omega_{\Gamma}^{\prime}}\right)$, which is obviously dependent of $\left\{\left(\Omega^{\lambda}\right)^{\prime}\right\}$ and $\Omega^{\prime}$, is independent of embeddings $\Gamma^{\lambda}$ from $\overline{\Omega^{\prime}}$ onto $\overline{\left(\Omega^{\lambda}\right)^{\prime}}$, provided there exists $\left\{b_{\lambda}\right\} \in \mathcal{C}^{j}([0,1])$ such that $\Gamma_{a, b}^{\lambda}$ extend to $\mathcal{C}^{1}$ embeddings from $\overline{\Omega_{a}}$ onto $\overline{\Omega_{b}^{\lambda}}$ for some $a \in \Omega$ and $\Gamma_{a, b} \in \mathcal{B}^{k+\alpha, j}\left(\overline{\Omega_{a}}\right) \cap \mathcal{C}^{1,0}\left(\overline{\Omega_{a}}\right)$. Finally, we always assume that $\gamma^{\lambda}$ are the restrictions of $\Gamma^{\lambda}$ on $\partial \Omega$, which preserve orientation.

Proposition 8.7. Let $k \geq j$ and $k+1 \geq l \geq 0$. Let $\Omega$ be a bounded and simply connected domain with $\partial \Omega \in \mathcal{C}^{k+1+\alpha}$ and let $\bar{\Gamma}^{\lambda}$ map $\overline{\Omega^{\prime}}$ onto $\overline{\left(\Omega^{\lambda}\right)^{\prime}}$ for $0 \leq \lambda \leq 1$. Let $b_{\lambda} \in \Omega^{\lambda}$ satisfy $\left\{b_{\lambda}\right\} \in \mathcal{C}^{j}([0,1])$ and let $a \in \Omega$. Suppose that $\Gamma_{a, b}^{\lambda}$ extend to $\mathcal{C}^{1}$ embeddings from $\overline{\Omega_{a}}$ onto $\overline{\Omega_{b}^{\lambda}}$ with $\Gamma_{a, b} \in \mathcal{C}^{1,0}\left(\overline{\Omega_{a}}\right)$.

(i) If $\Gamma_{b} \in \mathcal{B}^{l+\alpha, j}\left(\overline{\Omega^{\prime}}\right)$ and $f \in \mathcal{B}^{l+\alpha, j}\left(\partial \Omega_{\gamma}\right)$, then $\left\{\mathcal{C}_{-}^{\lambda} f\right\} \in \mathcal{B}^{l+\alpha, j}\left(\overline{\Omega_{\Gamma}^{\prime}}\right)$. The analogous assertion holds if $\mathcal{C}^{l+\alpha, j}$ substitutes for $\mathcal{B}^{l+\alpha, j}$.

(ii) If $\partial \Omega \in \mathcal{C}^{\omega}, \Gamma_{a, b} \in \mathcal{C}^{\omega}\left(\overline{\Omega_{a}} \times[0,1]\right)$ and $\left\{f \circ \Gamma^{\lambda} \circ \iota_{a}\right\} \in \mathcal{C}^{\omega}\left(\partial \Omega_{a} \times[0,1]\right)$, then $\left\{\mathcal{C}_{-}^{\lambda} f \circ \Gamma^{\lambda} \circ \iota_{a}\right\} \in \mathcal{C}^{\omega}\left(\overline{\Omega_{a}} \times[0,1]\right)$.

Proof. By our definition of orientations of boundaries, $\iota_{a}$ reverses the orientations of $\partial \Omega$ and $\partial \Omega_{a}$ for $a \in \Omega$. Let $b_{\lambda} \in \Omega^{\lambda}, z^{\lambda} \in \mathbf{C} \backslash \overline{\Omega^{\lambda}}$, and $z_{\lambda} \in \Omega_{b}^{\lambda}$. Applying the inversion $\iota_{b_{\lambda}}$ to replace $\zeta^{\lambda}-b_{\lambda}$ by $\left(\zeta_{\lambda}-b_{\lambda}\right)^{-1}$, we get

$$
\begin{gathered}
\mathcal{C}_{-}^{\lambda} f\left(z^{\lambda}\right)=-\frac{z_{\lambda}-b_{\lambda}}{2 \pi i} \int_{\partial \Omega_{b}^{\lambda}} \frac{\left(\zeta_{\lambda}-b_{\lambda}\right)^{-1} f^{\lambda}\left(\iota_{b_{\lambda}}\left(\zeta_{\lambda}\right)\right)}{\zeta_{\lambda}-z_{\lambda}} d \zeta_{\lambda}, \\
\left(\mathcal{C}_{-}^{\lambda} f\right) \circ \Gamma^{\lambda} \circ \iota_{a}(z)=-\frac{\Gamma_{a, b}^{\lambda}(z)-b_{\lambda}}{2 \pi i} \int_{\partial \Omega_{a}} \frac{\left(\Gamma_{a, b}^{\lambda}(\zeta)-b_{\lambda}\right)^{-1} f^{\lambda} \circ \Gamma^{\lambda} \circ \iota_{a}(\zeta)}{\Gamma_{a, b}^{\lambda}(\zeta)-\Gamma_{a, b}^{\lambda}(z)} d \Gamma_{a, b}^{\lambda}(\zeta) .
\end{gathered}
$$


We know that $\left\{f^{\lambda} \circ \iota_{b} \circ \Gamma_{a, b}^{\lambda}\right\}=\left\{f^{\lambda} \circ \gamma^{\lambda} \circ \iota_{a}\right\}$ is in $\mathcal{B}^{k+\alpha, j}\left(\partial \Omega_{a}\right)$ and $\mathcal{C}^{k+\alpha, j}\left(\partial \Omega_{a}\right)$, when $f$ is in $\mathcal{B}^{k+\alpha, j}\left(\partial \Omega_{\gamma}\right)$ and $\mathcal{C}^{k+\alpha, j}\left(\partial \Omega_{\gamma}\right)$, respectively. The lemma follows from Proposition 5.2 .

Proposition 8.8. Keep the assumptions in Proposition 8.7. Let $f \in \mathcal{C}_{*}^{0, j}\left(\partial \Omega_{\gamma}\right)$.

(i) Assume that $\int_{\partial \Omega^{\lambda}} f^{\lambda} d \sigma^{\lambda}=0$. If $\Gamma_{b} \in \mathcal{C}_{*}^{1, j}\left(\overline{\Omega^{\prime}} \Gamma\right)$, then $W_{-} f \in \mathcal{C}_{*}^{0, j}\left(\overline{\Omega^{\prime}} \Gamma\right)$. Assume that $\partial \Omega \in \mathcal{C}^{k+1+\alpha}, \Gamma_{b} \in \mathcal{B}^{k+1+\alpha, j}\left(\overline{\Omega^{\prime}} \Gamma\right)$ and $f \in \mathcal{B}^{k+\alpha, j}\left(\partial \Omega_{\gamma}\right)$. Then $W_{-} f \in \mathcal{B}^{k+1+\alpha, j}\left(\overline{\Omega^{\prime}} \Gamma\right)$. The analogous assertion holds if $\mathcal{C}$ substitutes for $\mathcal{B}$. Assume further that $\partial \Omega \in \mathcal{C}^{\omega},\left\{b_{\lambda}\right\} \in \mathcal{C}^{\omega}, \Gamma_{a, b} \in \mathcal{C}^{\omega}\left(\overline{\Omega_{a}} \times[0,1]\right)$ and $\left\{f^{\lambda} \circ \gamma^{\lambda}\right\} \in \mathcal{C}^{\omega}(\partial \Omega \times[0,1])$. Then $\left\{\left(W_{-}^{\lambda} f\right) \circ \Gamma^{\lambda} \circ \iota_{a}\right\} \in \mathcal{C}^{\omega}\left(\overline{\Omega_{a}} \times[0,1]\right)$.

(ii) If $\Gamma_{b} \in \mathcal{B}_{*}^{1+\alpha, j}\left(\overline{\Omega^{\prime}} \Gamma\right)$, then $U_{-} f \in \mathcal{C}_{*}^{0, j}\left(\overline{\Omega^{\prime}} \Gamma\right)$.

Proof. Let $A$ be an orientation preserving map from $\partial \hat{\Omega}$ onto $\partial \Omega$. Let $\hat{\gamma}(t)$ be a parameterization of $\partial \hat{\Omega}$. Then $\gamma(t)=A(\hat{\gamma}(t))$ is a parameterization of $\partial \Omega$. Assume that $d t$ agrees with the orientation of $\partial \Omega$ and $A$ extends to a $\mathcal{C}^{1}$ map defined near $\partial \Omega$. We have

$$
d \sigma=\left|\partial_{t} A(\hat{\gamma}(t))\right| d t=\left|\partial_{z} A+\hat{\gamma}^{\prime}(t)^{-1} \overline{\hat{\gamma}^{\prime}(t)} \partial_{\bar{z}} A\right| d \hat{\sigma} .
$$

Let $d \sigma_{b}^{\lambda}$ be the arc-length element on $\partial \Omega_{b}^{\lambda}$. Since $\iota_{b_{\lambda}}: z^{\lambda} \rightarrow z_{\lambda}$ reverses the orientations of $\partial \Omega^{\lambda}$ and $\partial \Omega_{b}^{\lambda}$, we obtain

$$
d \sigma^{\lambda}=-\frac{d \sigma_{b}^{\lambda}}{\left|\zeta_{\lambda}-b_{\lambda}\right|^{2}}
$$

on $\partial \Omega_{b}^{\lambda}$ or $\partial \Omega^{\lambda}$ (via pull-back or push-forward). By (3.2), a simple computation yields

$$
\begin{aligned}
W_{-}^{\lambda} f\left(z^{\lambda}\right)= & \frac{1}{\pi} \int_{\partial \Omega^{\lambda}} f^{\lambda}\left(\zeta^{\lambda}\right) \log \left|\zeta_{\lambda}-z_{\lambda}\right| d \sigma^{\lambda} \\
& -\frac{1}{\pi} \int_{\partial \Omega^{\lambda}} f^{\lambda}\left(\zeta^{\lambda}\right) \log \left|\left(\zeta_{\lambda}-b_{\lambda}\right)\left(z_{\lambda}-b_{\lambda}\right)\right| d \sigma^{\lambda}, \quad z_{\lambda} \neq b_{\lambda} .
\end{aligned}
$$

Since $\int_{\partial \Omega^{\lambda}} f^{\lambda} d \sigma^{\lambda}=0$, we can remove $\left(z_{\lambda}-b_{\lambda}\right)$ and the restriction $z_{\lambda} \neq b_{\lambda}$ from the last integral. By (8.23), we get

$$
\begin{aligned}
W_{-}^{\lambda} f\left(z^{\lambda}\right)= & -\frac{1}{\pi} \int_{\partial \Omega_{b}^{\lambda}} f^{\lambda}\left(\iota_{b_{\lambda}}\left(\zeta_{\lambda}\right)\right) \frac{1}{\left|\zeta_{\lambda}-b_{\lambda}\right|^{2}} \log \left|\zeta_{\lambda}-z_{\lambda}\right| d \sigma_{b}^{\lambda} \\
& +\frac{1}{\pi} \int_{\partial \Omega_{b}^{\lambda}} f^{\lambda}\left(\iota_{b_{\lambda}}\left(\zeta_{\lambda}\right)\right) \frac{1}{\left|\zeta_{\lambda}-b_{\lambda}\right|^{2}} \log \left|\zeta_{\lambda}-b_{\lambda}\right| d \sigma_{b}^{\lambda}, \quad z_{\lambda} \in \Omega_{b}^{\lambda}
\end{aligned}
$$

Let $\tau_{b, \zeta}^{\lambda}$ be the unit tangent vector of $\partial \Omega_{b}^{\lambda}$ at $\zeta_{\lambda}$. Fixing $z \in \Omega$, we have $d \arg \left(z^{\lambda}-\right.$ $\left.\zeta^{\lambda}\right)=\left(\partial_{\tau_{b, \zeta}^{\lambda}} \arg \left(\zeta_{\lambda}-z_{\lambda}\right)-\partial_{\tau_{b, \zeta}^{\lambda}} \arg \left(\zeta_{\lambda}-b_{\lambda}\right)\right) d \sigma_{b}^{\lambda}\left(\zeta_{\lambda}\right)$ and by (3.1)

$$
\begin{aligned}
U_{-}^{\lambda} f\left(z^{\lambda}\right)= & -\frac{1}{\pi} \int_{\partial \Omega_{b}^{\lambda}} f^{\lambda}\left(\iota_{b_{\lambda}}\left(\zeta_{\lambda}\right)\right) \partial_{\tau_{b, \zeta}^{\lambda}} \arg \left(\zeta_{\lambda}-z_{\lambda}\right) d \sigma_{b}^{\lambda}\left(\zeta_{\lambda}\right) \\
& +\frac{1}{\pi} \int_{\partial \Omega_{b}^{\lambda}} f^{\lambda}\left(\iota_{b_{\lambda}}\left(\zeta_{\lambda}\right)\right) \partial_{\tau_{b, \zeta}^{\lambda}} \arg \left(\zeta_{\lambda}-b_{\lambda}\right) d \sigma_{b}^{\lambda}\left(\zeta_{\lambda}\right), \quad z_{\lambda} \in \Omega_{b}^{\lambda} .
\end{aligned}
$$

The assertions follow from Proposition 5.3 and the last two formulae. 


\section{MAIN RESUlts AND PROOFS}

In this section, we first prove the real analyticity of solutions to real analytic integral equations arising from the Dirichlet and Neumann problems. We then collect results from previous sections to formulate the solutions of Dirichlet and Neumann problems with parameter. Finally, we prove Theorem 1.2

Proposition 9.1. Let $\Omega$ be a bounded domain with $\partial \Omega \in \mathcal{C}^{\omega}$. Let $\gamma^{\lambda}$ embed $\partial \Omega$ onto $\partial \Omega^{\lambda}$ with $\gamma \in \mathcal{C}^{\omega}(\partial \Omega \times[0,1])$. Let $\mathcal{L}$ be one of $K,-K, K^{*}$, and $-K^{*}$. Let $\psi \in \mathcal{C}^{\omega}(\partial \Omega \times[0,1])$. Suppose that $\varphi^{\lambda} \in L^{1}\left(\partial \Omega^{\lambda}\right)$ satisfy

$$
\varphi^{\lambda}+\mathcal{L}^{\lambda} \varphi^{\lambda}=\psi^{\lambda}, \quad\left\{\left\langle\varphi^{\lambda}, \ell_{j}^{\lambda}\right\rangle\right\} \in \mathcal{C}^{\omega}, \quad 1 \leq j \leq n .
$$

Then $\varphi \in \mathcal{C}^{\omega}(\partial \Omega \times[0,1])$. Furthermore, the functions $\phi_{0}, \ldots, \phi_{m}$ in Proposition 6.2 are in $\mathcal{C}^{\omega}(\partial \Omega \times[0,1])$.

Proof. We already know that $\varphi, \phi_{i}$ are of class $\mathcal{C}^{\infty}$. We apply Cauchy majorant methods to estimate the growth of their Taylor coefficients. By Taylor's theorem, a function $f$ on $\partial \Omega \times[0,1]$ is real analytic if and only if

$$
\max _{t, \lambda}\left|\partial_{t}^{i} \partial_{\lambda}^{j} f(\hat{\gamma}(t), \lambda)\right| \leq C i ! j ! \rho^{i+j},
$$

where $\hat{\gamma}$ is a real analytic parameterization of $\partial \Omega$ and $C, \rho$ are constants. We first need uniform bounds for solution operators in sup-norms. Let $\left\{\ell_{1}^{\lambda}, \ell_{2}^{\lambda}, \ldots, \ell_{n}^{\lambda}\right\}$ be the basis of $\operatorname{ker}\left(I+\mathcal{L}^{\lambda}\right)$ described after the proof of Proposition 6.2. By Lemma 7.1 (i), we know that $\ell_{1}, \ldots, \ell_{n}$ are in $\mathcal{C}^{0,0}\left(\partial \Omega_{\gamma}\right)$. Then $\mathcal{L}_{0}$ sends $\mathcal{C}^{0,0}\left(\partial \Omega_{\gamma}\right)$ into $\left(\mathcal{C}^{0}([0,1])\right)^{n}$, where

$$
\mathcal{L}_{0}^{\lambda} \varphi=\left(\left\langle\varphi^{\lambda}, \ell_{1}^{\lambda}\right\rangle,\left\langle\varphi^{\lambda}, \ell_{2}^{\lambda}\right\rangle, \ldots,\left\langle\varphi^{\lambda}, \ell_{n}^{\lambda}\right\rangle\right) .
$$

Consider bounded linear maps

$$
\begin{gathered}
\left(I+\mathcal{L}, \mathcal{L}_{0}\right): \mathcal{C}^{0,0}\left(\partial \Omega_{\gamma}\right) \rightarrow\left(C^{0,0}\left(\partial \Omega_{\gamma}\right) \cap \operatorname{ker}\left(I+\mathcal{L}^{*}\right)^{\perp}\right) \times\left(\mathcal{C}^{0}([0,1])\right)^{n}=X_{\mathcal{L}} \\
\left(I+\mathcal{L}^{*}, \mathcal{L}_{0}\right): \mathcal{C}^{0,0}\left(\partial \Omega_{\gamma}\right) \rightarrow X_{\mathcal{L}^{*}}, \quad \mathcal{L}=\mathcal{K} \text { or }-\mathcal{K} .
\end{gathered}
$$

It is clear that $\left(I+\mathcal{L}, \mathcal{L}_{0}\right)$ is injective. By Proposition 6.2 (iii), the second map is also injective for both cases. By Proposition 6.2 (i) and Lemma 7.1 (i), $I+\mathcal{L}$ maps $\mathcal{C}^{0,0}\left(\partial \Omega_{\gamma}\right)$ onto $\mathcal{C}^{0,0} \cap\left(\operatorname{ker}\left(I+\mathcal{L}^{*}\right)\right)^{\perp}$. Since $\ell_{1}^{\lambda}, \ldots, \ell_{n}^{\lambda}$ are linearly independent for each $\lambda$, then $\left(\left\langle\ell_{i}^{\lambda}, \ell_{j}^{\lambda}\right\rangle\right)_{1 \leq i, j \leq n}$ are invertible. Since $\ell_{i}$ are in $\mathcal{C}^{0,0}\left(\partial \Omega_{\gamma}\right)$, given $c \in\left(\mathcal{C}^{0}([0,1])\right)^{n}$ we can find $\tilde{c} \in\left(\mathcal{C}^{0}([0,1])\right)^{n}$ such that $\left\langle\sum_{j} \tilde{c}_{j}(\lambda) \ell_{j}^{\lambda}, \ell_{i}^{\lambda}\right\rangle=c_{i}(\lambda)$. This shows that $\left(I+\mathcal{L}, \mathcal{L}_{0}\right)$ is surjective. That $\left(I+\mathcal{L}^{*}, \mathcal{L}_{0}\right)$ is surjective for $\mathcal{L}=\mathcal{K}$ or $-\mathcal{K}$ follows from $\int_{\partial \Omega^{\lambda}} e_{i} \phi_{j}^{\lambda} d \sigma^{\lambda}=\delta_{i j}$ for $1 \leq i, j \leq m$, and $\int_{\partial \Omega^{\lambda}} e_{0} \phi_{0}^{\lambda} d \sigma^{\lambda}=1$. By the open mapping theorem, we have

$$
\begin{gathered}
|\varphi|_{0,0} \leq C_{*}\left(\left|\left(I+\mathcal{L}^{*}\right) \varphi\right|_{0,0}+\left|\mathcal{L}_{0} \varphi\right|_{0}\right), \quad \mathcal{L}=\mathcal{K} \text { or }-\mathcal{K} \\
|\varphi|_{0,0} \leq C_{*}\left(|(I+\mathcal{L}) \varphi|_{0,0}+\left|\mathcal{L}_{0} \varphi\right|_{0}\right), \quad \mathcal{L}=\mathcal{K},-\mathcal{K}, \mathcal{K}^{*}, \text { or }-\mathcal{K} .
\end{gathered}
$$

Here the $\varphi$ is in $\mathcal{C}^{0,0}\left(\partial \Omega_{\gamma}\right)$ and $C_{*}$ is independent of $\varphi$.

(i) We first consider the case where $\mathcal{L}=\mathcal{K}$ or $-\mathcal{K}$. We express (9.1) as

$$
\begin{aligned}
& \varphi(z, \lambda)+\int_{\partial \Omega} \varphi(\zeta, \lambda) L(z, \zeta, \lambda) d \sigma(\zeta)=\psi_{0}(z, \lambda), \\
& \int_{\partial \Omega} \varphi(\zeta, \lambda) \ell_{\alpha}^{\lambda} a(\zeta, \lambda) d \sigma(\zeta)=\psi_{\alpha}^{\lambda}, \quad \alpha=1, \ldots, n .
\end{aligned}
$$


Note that $L(z, \zeta, \lambda)$ is real analytic on $\partial \Omega \times \partial \Omega \times[0,1]$ and $a(\zeta, \lambda)=\partial_{\tau^{\lambda}} \gamma^{\lambda}(\zeta)$ is real analytic on $\partial \Omega \times[0,1]$. We know that $\ell_{i}$ are locally constants. However, we want to reason in such a way that the proof is valid whenever $\ell_{i}^{\lambda}(\zeta)$ are real analytic in $\lambda$ and $\zeta$. Thus, the proof applies to $\mathcal{L}=\mathcal{K}^{*}$ or $-\mathcal{K}^{*}$ after we prove (ii). Differentiating (9.4)-(9.5) yields

$$
\begin{aligned}
& \partial_{\lambda}^{k} \varphi(z, \lambda)+\mathcal{L}^{\lambda} \partial_{\lambda}^{k} \varphi^{\lambda}=\partial_{\lambda}^{k} \psi_{0}(z, \lambda) \\
&-\sum_{l=0}^{k-1}\left(\begin{array}{l}
k \\
l
\end{array}\right) \int_{\partial \Omega} \partial_{\lambda}^{l} \varphi(\zeta, \lambda) \partial_{\lambda}^{k-l} L(z, \zeta, \lambda) d \sigma(\zeta), \\
& \mathcal{L}_{0} \partial_{\lambda}^{k} \varphi^{\lambda}=\partial_{\lambda}^{k} \psi(\lambda)-\sum_{l=0}^{k-1}\left(\begin{array}{l}
k \\
l
\end{array}\right) \int_{\partial \Omega} \partial_{\lambda}^{l} \varphi(\zeta, \lambda) \partial_{\lambda}^{k-l}\left(\ell^{\lambda}(\zeta) a(\zeta, \lambda)\right) d \sigma(\zeta) .
\end{aligned}
$$

Set $A_{k}=\frac{1}{k !} \max _{\zeta, \lambda}\left|\partial_{\lambda}^{k} \varphi(\zeta, \lambda)\right|$ and

$$
a_{k}=\frac{1}{k !} \max _{\zeta, z, \lambda, \alpha}\left\{\left|\partial_{\lambda}^{k} L(\zeta, z, \lambda)\right|,\left|\partial_{\lambda}^{k} \psi_{0}(\zeta, \lambda)\right|,\left|\partial_{\lambda}^{k} \psi_{\alpha}(\lambda)\right|,\left|\partial_{\lambda}^{k}\left(\ell_{\alpha}^{\lambda} a(\zeta, \lambda)\right)\right|\right\} .
$$

We have $\left|\ell_{\alpha}\right|{ }_{L^{2}}^{-2}\left|\ell_{\alpha}\right| \leq C_{1}$. Denote by $|\partial \Omega|$ the length of $\partial \Omega$. Then we obtain from (9.7), (9.3) and (9.6)

$$
\frac{1}{k !}\left|\mathcal{L}_{0}^{\lambda} \partial_{\lambda}^{k} \varphi^{\lambda}\right| \leq a_{k}+|\partial \Omega| \sum_{l=0}^{k-1} A_{l} a_{k-l}, \quad A_{k} \leq 2 C_{*}\left\{a_{k}+|\partial \Omega| \sum_{l=0}^{k-1} A_{l} a_{k-l}\right\} .
$$

Denote $\sum A_{I} w^{I} \prec \sum b_{I} w^{I}$ if $A_{I} \leq b_{I}$ for $|I| \geq 0$. The above implies that

$$
\sum A_{k} w^{k} \prec 2 C_{*} \sum a_{k} w^{k}+2 C_{*}|\partial \Omega| w \sum A_{k} w^{k} \sum a_{k+1} w^{k} .
$$

Therefore, $\sum A_{k} w^{k}$ converges near the origin. Set $B_{k j}=\frac{1}{k ! j !} \max _{t, \lambda}\left|\partial_{t}^{j} \partial_{\lambda}^{k} \varphi(\hat{\gamma}(t), \lambda)\right|$ and

$$
b_{k j}=\frac{1}{k ! j !} \max _{\zeta, t, \lambda}\left(\left|\partial_{t}^{j} \partial_{\lambda}^{k} L(\hat{\gamma}(t), \zeta, \lambda)\right|,\left|\partial_{t}^{j} \partial_{\lambda}^{k} \psi_{0}(\hat{\gamma}(t), \lambda)\right|\right) .
$$

Taking $\partial_{t}^{j}$ directly onto the real analytic kernel $\partial_{\lambda}^{k} L(\hat{\gamma}(t), \zeta, \lambda)$ in (9.6), we get

$$
\begin{gathered}
\partial_{t}^{j} \partial_{\lambda}^{k} \varphi(\hat{\gamma}(t), \lambda)=\partial_{t}^{\lambda} \partial_{\lambda}^{k} \psi_{0}(\hat{\gamma}(t), \lambda)-\sum_{l=0}^{k}\left(\begin{array}{l}
k \\
l
\end{array}\right) \int_{\partial \Omega} \partial_{\lambda}^{l} \varphi(\zeta, \lambda) \partial_{t}^{j} \partial_{\lambda}^{k-l} L(\hat{\gamma}(t), \zeta, \lambda) d \sigma(\zeta), \\
B_{k j} \leq b_{k j}+|\partial \Omega| \sum_{l=0}^{k} A_{l} b_{(k-l) j}, \quad k, j \geq 0, \\
\sum B_{k j} w_{1}^{k} w_{2}^{j} \prec \sum b_{k j} w_{1}^{k} w_{2}^{j}+|\partial \Omega| \sum A_{k} w_{1}^{k} \sum b_{k j} w_{1}^{k} w_{2}^{j} .
\end{gathered}
$$

Obviously, $\sum B_{k j} t^{j} \lambda^{k}$ converges near $(t, \lambda)=0$.

(ii) We still consider $L=K$ or $-K$. The elements in the base $\left\{\phi_{i}^{\lambda}\right\}$ of $\operatorname{ker}\left(I+\mathcal{L}^{*}\right)$ are not constant, so we need to first establish their analyticity. Recall that

$$
\phi_{i}^{\lambda}+\left(\mathcal{L}^{\lambda}\right)^{*} \phi_{i}^{\lambda}=0, \quad \int_{\partial \Omega} \phi_{i}^{\lambda} \ell_{j}^{\lambda} a(\lambda, \zeta) d \sigma=\delta_{i j}, \quad 1 \leq i, j \leq n .
$$


We write both in vectors and matrices and get

$$
\begin{array}{r}
\left(I+\left(\mathcal{L}^{\lambda}\right)^{*}\right) \partial_{\lambda}^{k} \phi(z, \lambda)=-\sum_{l=0}^{k-1}\left(\begin{array}{l}
k \\
l
\end{array}\right) \int_{\partial \Omega} \partial_{\lambda}^{l} \phi(\zeta, \lambda) \partial_{\lambda}^{k-l} L(\zeta, z, \lambda) d \sigma(\zeta), \\
\mathcal{L}_{0}^{\lambda} \partial_{\lambda}^{k} \phi^{\lambda}=\partial_{\lambda}^{k} \operatorname{diag}(1, \ldots, 1)-\sum_{l=0}^{k-1}\left(\begin{array}{l}
k \\
l
\end{array}\right) \int_{\partial \Omega} \partial_{\lambda}^{l} \phi(\zeta, \lambda) \partial_{\lambda}^{k-l}\left(\ell^{\lambda} a(\zeta, \lambda)\right) d \sigma(\zeta) .
\end{array}
$$

We use (9.2) instead of (9.3) and get, for $A_{k}=\frac{1}{k !} \max _{\zeta, 1 \leq \lambda \leq n}\left|\partial_{\lambda}^{k} \phi_{\alpha}(\zeta, \lambda)\right|$,

$$
\frac{1}{k !}\left|\mathcal{L}_{0}^{\lambda} \partial_{\lambda}^{k} \phi^{\lambda}\right| \leq 1+|\partial \Omega| \sum_{l=0}^{k-1} A_{l} a_{k-l}, \quad A_{k} \leq 2 C_{*}\left\{1+|\partial \Omega| \sum_{l=0}^{k-1} A_{l} a_{k-l}\right\} .
$$

Therefore, $\sum A_{k} w^{k} \prec 2 C_{*}+2 C_{*}|\partial \Omega| w \sum A_{k} w^{k} \sum a_{k+1} w^{k}$ and $\sum A_{k} w^{k}$ converges near the origin. Next, we apply $\partial_{t}^{j}$ to (9.8) and get

$$
\partial_{t}^{j} \partial_{\lambda}^{k} \phi(\hat{\gamma}(t), \lambda)=-\sum_{l=0}^{k}\left(\begin{array}{l}
k \\
l
\end{array}\right) \int_{\partial \Omega} \partial_{\lambda}^{l} \phi(\zeta, \lambda) \partial_{t}^{j} \partial_{\lambda}^{k-l} L(\zeta, \hat{\gamma}(t), \lambda) d \sigma(\zeta) .
$$

As before, we obtain real analyticity of $\phi(\hat{\gamma}(t), \lambda)$.

With the real analyticity of $\phi_{i}$, the proof in (i) is valid for $\mathcal{L}=\mathcal{K}^{*}$ or $-\mathcal{K}^{*}$.

The Dirichlet problem for exterior domains with parameter is

$$
\Delta u^{\lambda}=0 \quad \text { on }\left(\Omega^{\lambda}\right)^{\prime}, \quad u^{\lambda}=f^{\lambda} \quad \text { on } \partial \Omega^{\lambda} .
$$

To ensure that the solutions are unique, we require that $u^{\lambda}$ be harmonic at $\infty$, i.e., that $u^{\lambda}(1 / z)$ is harmonic in a neighborhood of 0 . The Neumann problem for exterior domains with parameter is

$$
\Delta v^{\lambda}=0 \quad \text { on }\left(\Omega^{\lambda}\right)^{\prime}, \quad \partial_{\nu^{\lambda}} v^{\lambda}=g^{\lambda} \quad \text { on } \partial \Omega^{\lambda} .
$$

Here $\nu^{\lambda}$ is the unit outer normal vector of $\partial \Omega^{\lambda}$. Again, we require that $v^{\lambda}$ be harmonic at $\infty$. For the existence and uniqueness of solutions $v^{\lambda}$, we impose conditions

$$
\int_{\gamma_{i}^{\lambda}} g^{\lambda} d \sigma^{\lambda}=0, \quad \int_{\gamma_{i}^{\lambda}} v^{\lambda} d \sigma^{\lambda}=0, \quad 0 \leq i \leq m .
$$

The $v^{\lambda}$ which satisfy conditions (1.3) or (9.9) are called normalized solutions. By Hopf's lemma, if $u$ is harmonic on $\Omega$ and continuous up to the boundary with $\partial \Omega \in \mathcal{C}^{1+\alpha}$, then $\partial_{\nu} u$ determines $u$ up to a constant. In fact, one can locally reduce to the case where $\Omega$ is a unit disc by Kellogg's theorem; see also [10], p. 7. Thus, the normalized solutions are unique.

Recall that function spaces for interior domains are defined in section 2 and function spaces for exterior domains are defined in section 8 . The reader is referred to Lemma 2.2 for independence of spaces $\mathcal{B}^{k+\beta, j}\left(\partial \Omega_{\gamma}\right)$ and $\mathcal{B}^{k+\beta, j}\left(\partial \Omega_{\Gamma}\right)$ on $\gamma$ and $\Gamma$ for $k \geq j$, respectively. Recall that Lemma 8.6 shows the existence of extensions of $\gamma^{\lambda}$ to $\Gamma^{\lambda}$. We now summarize the solutions to the Dirichlet and Neumann problems with parameter as follows.

Theorem 9.2. Let $0 \leq j \leq k, 0<\alpha<1$, and $j \leq l \leq k+1$. Let $\Omega$ be a connected bounded domain in $\mathbf{C}$ with $\partial \Omega \in \mathcal{C}^{k+1+\alpha}$. Let $\Gamma^{\lambda}$ embed $\bar{\Omega}$ onto $\overline{\Omega^{\lambda}}$ with $\Gamma \in \mathcal{B}^{k+1+\alpha, j}(\bar{\Omega})$ for interior Dirichlet and Neumann problems. Let $\Gamma^{\lambda}$ embed $\overline{\Omega^{\prime}}$ onto $\overline{\left(\Omega^{\lambda}\right)^{\prime}}$ such that $\iota_{b^{\lambda}} \circ \Gamma^{\lambda} \circ \iota_{a}$ extends to $\mathcal{C}^{1}$ embeddings from $\overline{\Omega_{a}}$ onto $\overline{\Omega_{b}^{\lambda}}$ with $\Gamma_{b} \in \mathcal{B}^{k+1+\alpha, j}\left(\overline{\Omega_{\Gamma}^{\prime}}\right)$ for exterior Dirichlet and Neumann problems. Here $a \in \Omega$, 
$b_{\lambda} \in \Omega^{\lambda}$ and $\left\{b_{\lambda}\right\} \in \mathcal{C}^{j}([0,1])$. Let $\gamma^{\lambda}$ be the restriction of $\Gamma^{\lambda}$ on $\partial \Omega$. Suppose that $\left\{f^{\lambda}\right\} \in \mathcal{C}_{*}^{0, j}\left(\partial \Omega_{\gamma}\right)$.

( $i)$ (Interior Dirichlet problem.) There exists a unique harmonic function $u^{\lambda}$ on $\Omega^{\lambda}$ such that $u \in \mathcal{C}_{*}^{0, j}\left(\bar{\Omega}_{\Gamma}\right)$ and $u^{\lambda}=f^{\lambda}$ on $\partial \Omega^{\lambda}$. Moreover,

$$
\begin{gathered}
u^{\lambda}=U_{+}^{\lambda} \varphi+\sum_{i, j=1}^{m} c_{i}^{\lambda} \mu_{i j}^{\lambda} W_{+}^{\lambda} \phi_{j}, \\
\varphi^{\lambda}+\mathcal{K}^{\lambda} \varphi^{\lambda}=g^{\lambda}, \quad \varphi^{\lambda} \perp \operatorname{ker}\left(I+\mathcal{K}^{\lambda}\right), \quad g^{\lambda}=f^{\lambda}-\sum_{j=1}^{m} c_{i}^{\lambda} e_{i}, \\
\left(\left.W^{\lambda} \phi_{i}\right|_{\gamma_{j}^{\lambda}}\right)_{1 \leq i, j \leq m}=\left(\mu_{i j}^{\lambda}\right)^{-1}, \quad c_{i}^{\lambda}=\int_{\partial \Omega^{\lambda}} f^{\lambda} \phi_{i}^{\lambda} d \sigma^{\lambda} .
\end{gathered}
$$

(ii) (Exterior Dirichlet problem.) Assume that $\Omega^{\lambda}$ are simply connected. There exists a unique harmonic function $u^{\lambda}$ on $\left(\Omega^{\lambda}\right)^{\prime} \cup\{\infty\}$ such that $u \in$ $\mathcal{C}_{*}^{0, j}\left(\overline{\Omega_{\Gamma}^{\prime}}\right)$ and $u^{\lambda}=f^{\lambda}$ on $\partial \Omega^{\lambda}$. Moreover,

$$
\begin{gathered}
u^{\lambda}=U_{-}^{\lambda} \varphi+\int_{\partial \Omega^{\lambda}} f^{\lambda} \phi_{0}^{\lambda} d \sigma^{\lambda}, \\
\varphi^{\lambda}-\mathcal{K}^{\lambda} \varphi^{\lambda}=g^{\lambda}, \quad \varphi^{\lambda} \perp \operatorname{ker}\left(I-\mathcal{K}^{\lambda}\right), \quad g^{\lambda}=f^{\lambda}-\int_{\partial \Omega^{\lambda}} f^{\lambda} \phi_{0}^{\lambda} d \sigma^{\lambda} .
\end{gathered}
$$

(iii) (Interior Neumann problem.) Let $\left|\partial \Omega^{\lambda}\right|$ be the arc length of $\partial \Omega^{\lambda}$. Assume that $\int_{\partial \Omega^{\lambda}} f^{\lambda} d \sigma^{\lambda}=0$. There exist $u^{\lambda}$ which are harmonic on $\Omega^{\lambda}$ and satisfy $\left\{u^{\lambda}\right\} \in \mathcal{C}_{*}^{0, j}\left(\bar{\Omega}_{\Gamma}\right)$ and $\partial_{\nu^{\lambda}} u^{\lambda}=f^{\lambda}$. The normalized solutions $u^{\lambda}$ are given by

$$
\begin{gathered}
\varphi^{\lambda}-\left(\mathcal{K}^{\lambda}\right)^{*} \varphi^{\lambda}=f^{\lambda}, \quad \varphi^{\lambda} \perp \operatorname{ker}\left(I-\left(\mathcal{K}^{\lambda}\right)^{*}\right), \\
u^{\lambda}=W_{+}^{\lambda} \varphi-\frac{1}{\left|\partial \Omega^{\lambda}\right|} \int_{\partial \Omega^{\lambda}} W_{+}^{\lambda} \varphi d \sigma^{\lambda} .
\end{gathered}
$$

(iv) (Exterior Neumann problem.) Assume that $\Omega^{\lambda}$ are simply connected and $\int_{\gamma_{j}^{\lambda}} f^{\lambda} d \sigma^{\lambda}=0$ for all $j \geq 0$. There exist functions $u^{\lambda}$ which are harmonic on $\left(\Omega^{\lambda}\right)^{\prime} \cup\{\infty\}$ and satisfy $u \in \mathcal{C}_{*}^{0, j}\left(\overline{\Omega_{\Gamma}^{\prime}}\right)$ and $\partial_{\nu^{\lambda}} u^{\lambda}=f^{\lambda}$. The normalized solutions are $u^{\lambda}$ given by

$$
\begin{gathered}
\varphi^{\lambda}+\left(\mathcal{K}^{\lambda}\right)^{*} \varphi^{\lambda}=f^{\lambda}, \quad \varphi^{\lambda} \perp \operatorname{ker}\left(I+\left(\mathcal{K}^{\lambda}\right)^{*}\right), \\
u^{\lambda}=W_{-}^{\lambda} \varphi-\frac{1}{\left|\partial \Omega^{\lambda}\right|} \int_{\partial \Omega^{\lambda}} W_{-}^{\lambda} \varphi d \sigma^{\lambda} .
\end{gathered}
$$

(v) (Regularity.) If $f \in \mathcal{B}^{l+\alpha, j}\left(\partial \Omega_{\gamma}\right)$, then $u \in \mathcal{B}^{l+\alpha, j}\left(\bar{\Omega}_{\Gamma}\right)$ for $(i)$ and $u \in$ $\mathcal{B}^{l+\alpha, j}\left({\overline{\Omega^{\prime}}}_{\Gamma}\right)$ for $($ ii $)$; if $f \in \mathcal{B}^{k+\alpha, j}\left(\partial \Omega_{\gamma}\right)$ then $u \in \mathcal{B}^{k+1+\alpha, j}\left(\bar{\Omega}_{\Gamma}\right)$ for $($ iii $)$ and $u \in \mathcal{B}^{k+1+\alpha, j}\left(\overline{\Omega^{\prime}} \Gamma\right)$ for $(i v)$. Assume further that $\Gamma \in \mathcal{C}^{k+1+\alpha, j}(\bar{\Omega})$ and $\Gamma_{b} \in \mathcal{C}^{k+1+\alpha, j}(\bar{\Omega})$. If $f \in \mathcal{C}^{l+\alpha, j}\left(\partial \Omega_{\gamma}\right)$, then $u \in \mathcal{C}^{l+\alpha, j}\left(\bar{\Omega}_{\Gamma}\right)$ for $(i)$ and $u \in \mathcal{C}^{l+\alpha, j}\left(\overline{\Omega^{\prime}} \Gamma\right)$ for $(i i)$; if $f \in \mathcal{C}^{k+\alpha, j}\left(\partial \Omega_{\gamma}\right)$, then $u \in \mathcal{C}^{k+1+\alpha, j}\left(\bar{\Omega}_{\Gamma}\right)$ for (iii) and $u \in \mathcal{C}^{k+1+\alpha, j}\left(\overline{\Omega^{\prime}} \Gamma\right)$ for $(i v)$. Assume further that $\partial \Omega \in \mathcal{C}^{\omega}$, $\Gamma \in \mathcal{C}^{\omega}(\bar{\Omega} \times[0,1]), \Gamma_{a, b} \in \mathcal{C}^{\omega}\left(\overline{\Omega_{a}} \times[0,1]\right),\left\{b^{\lambda}\right\} \in \mathcal{C}^{\omega}([0,1])$, and $f \circ \gamma \in$ $\mathcal{C}^{\omega}(\partial \Omega \times[0,1])$. Then $u^{\lambda} \circ \Gamma^{\lambda}(z)$ is in $\mathcal{C}^{\omega}(\bar{\Omega} \times[0,1])$ for $(i)$ and $($ iii $)$, and $u^{\lambda} \circ \Gamma^{\lambda} \circ \iota_{a}(z)$ is in $\mathcal{C}^{\omega}\left(\overline{\Omega_{a}} \times[0,1]\right)$ for $(i i)$ and $(i v)$. 
Proof. For smoothness in parameter, we need to compute the coefficients in the solution formulae. We recall results from Proposition 6.2 We have $e_{i}=1$ on $\gamma_{i}$ and $e_{i}=0$ on $\partial \Omega^{\lambda} \backslash \gamma_{i}^{\lambda}$ for $i>0$, and $e_{0}=1$ on $\partial \Omega^{\lambda}$. Also $\left(\int_{\gamma_{j}^{\lambda}} \phi_{i}^{\lambda} d \sigma^{\lambda}\right)_{1 \leq i, j \leq m}=I$, $\int_{\partial \Omega^{\lambda}} \phi_{0}^{\lambda} e_{0} d \sigma^{\lambda}=1$ and $\phi_{0}=0$ on $\gamma_{i}^{\lambda}$ for $i>0$. We also know that, on $\partial \Omega^{\lambda}, W_{+}^{\lambda} \phi_{0}$ is constant and $W_{-}^{\lambda} \phi_{i}$ are locally constant for $i>0$. On $\partial \Omega^{\lambda}$ and for $i>0$, we have

$$
W_{-}^{\lambda} \phi_{i}=\sum_{j>0} \nu_{i j}^{\lambda} e_{j}, \quad \nu_{i j}^{\lambda}=\left.W_{-}^{\lambda} \phi_{i}\right|_{\gamma_{j}^{\lambda}}, \quad \operatorname{det}\left(\nu_{i j}^{\lambda}\right)_{1 \leq i, j \leq m} \neq 0 .
$$

(The latter needs $m>0$.) Thus for $j>0$ we have $e_{j}=\sum_{i=1}^{m} \mu_{j i} W_{-}^{\lambda} \phi_{i}$. By Proposition 7.4 a), we know that $\phi_{0}, \phi_{1}, \ldots, \phi_{m}$ are in $\mathcal{B}^{k+\alpha, j}\left(\partial \Omega_{\gamma}\right)$. Thus, $\nu_{i l}$ and $\mu_{i l}$ are in $\mathcal{C}^{j}([0,1])$. Let $c_{i}^{\lambda}=\int_{\partial \Omega^{\lambda}} f^{\lambda} \phi_{j}^{\lambda} d \sigma^{\lambda}$. Then $c_{i} \in \mathcal{C}^{j}([0,1])$ and

$f^{\lambda}=g^{\lambda}+c_{1}^{\lambda} e_{1}+\cdots+c_{m}^{\lambda} e_{m}, g^{\lambda} \perp \operatorname{ker}\left(I+\left(\mathcal{K}^{\lambda}\right)^{*}\right) ; \quad f^{\lambda}=g^{\lambda}+c_{0}^{\lambda}, g^{\lambda} \perp \operatorname{ker}\left(I-\left(\mathcal{K}^{\lambda}\right)^{*}\right)$.

It is clear that $g_{i} \in \mathcal{C}_{*}^{0, j}\left(\partial \Omega_{\gamma}\right)$. By Proposition 6.2 (i) and Lemma 7.3 c), we get $\varphi \in \mathcal{C}_{*}^{0, j}\left(\partial \Omega_{\gamma}\right)$ for (i)-(iv).

For (i) and (ii) with $f \in \mathcal{B}^{l+\alpha, j}\left(\partial \Omega_{\gamma}\right)$ and $l \leq k+1$, we still have $g \in \mathcal{B}^{l+\alpha, j}\left(\partial \Omega_{\gamma}\right)$ as $f-g \in \mathcal{C}^{\infty, j}\left(\partial \Omega_{\gamma}\right)$. Thus, $\varphi \in \mathcal{B}^{l+\alpha, j}\left(\partial \Omega_{\gamma}\right)$ by Proposition $\left.7.4 \mathrm{c}\right)$. Hence, $U_{+} \varphi=2 \operatorname{Re} \mathcal{C} \varphi \in \mathcal{B}^{l+\alpha, j}\left(\bar{\Omega}_{\Gamma}\right)$ by Proposition 5.2 and $U_{-} \varphi=2 \operatorname{Re} \mathcal{C} \varphi \in \mathcal{B}^{l+\alpha, j}\left(\bar{\Omega}_{\Gamma}\right)$ by Proposition 8.7. Also, $W_{+} \phi_{i} \in \mathcal{B}^{k+1+\alpha, j}\left(\bar{\Omega}_{\Gamma}\right)$ by Proposition 5.2 and $W_{-} \phi_{i} \in$ $\mathcal{B}^{k+1+\alpha, j}\left({\overline{\Omega^{\prime}}}_{\Gamma}\right)$ by Proposition 8.8. The coefficients $c_{i}, \mu_{i l}$ in (9.10) are in $\mathcal{C}^{\infty, j}$. We conclude that $u \in \mathcal{B}^{l+\alpha, j}\left(\bar{\Omega}_{\Gamma}\right)$ for (i) and $u \in \mathcal{B}^{l+\alpha, j}\left({\overline{\Omega^{\prime}}}_{\Gamma}\right)$ for (ii).

For (iii) and (iv) with $f \in \mathcal{B}^{k+\alpha, j}$, we get $\varphi \in \mathcal{B}^{k+\alpha, j}\left(\partial \Omega_{\gamma}\right)$ by Proposition 7.4 b). Hence, $W_{+} \varphi \in \mathcal{B}^{k+1+\alpha, j}\left(\bar{\Omega}_{\Gamma}\right)$ by Proposition 5.2 and $W_{-} \varphi \in \mathcal{B}^{k+1+\alpha, j}\left({\overline{\Omega^{\prime}}}_{\Gamma}\right)$ by Proposition 8.8

Finally, the real analytic results follow from Proposition 9.1, Proposition 8.8, Proposition 5.2. Proposition 8.7. and the solution formulae of the Dirichlet and Neumann problems.

Corollary 9.3. Let $k \geq 0$ be an integer and let $0<\beta<\alpha<1$. Let $\Omega$ be a bounded domain with $\partial \Omega \in \mathcal{C}^{k+1+\alpha}$. Let $f \in \mathcal{C}^{k+1+\beta}(\partial \Omega) \backslash \mathcal{C}^{k+1+\alpha}(\partial \Omega)$. Then $W f$ defines two harmonic functions on $\Omega$ and $\Omega^{\prime}$, which have the same boundary value. $\left.W f\right|_{\partial \Omega}$ is in $\mathcal{C}^{k+1+\beta}$, but not in $\mathcal{C}^{k+1+\alpha}$. Moreover, $W f \in \mathcal{C}^{1-\epsilon}(\mathbf{C})$ for any $\epsilon>0$.

As observed in [2, if the above $W f$ is in $\mathcal{C}^{1}(\mathbf{C})$, then (3.4) implies that $f$ and $W f$ are zero. It is trivial that if a continuous function is holomorphic on both sides of a real curve in the complex plane, the function is holomorphic near the curve. The reader is referred to [2] where regularities for functions for two-sided almost complex structures are in contrast to Corollary 9.3

As a consequence of Theorem 9.2, we have the following version of Kellogg's Riemann mapping theorem with parameter.

Corollary 9.4. Let $j, k$ be non-negative integers or $\infty$ satisfying $0 \leq j \leq k$. Let $0<$ $\alpha<1$. Let $\Omega$ be a simply connected bounded domain in $\mathbf{C}$ with $\partial \Omega \in \mathcal{C}^{k+1+\alpha}$ and let $\Gamma^{\lambda}$ embed $\bar{\Omega}$ onto $\overline{\Omega^{\lambda}}$ and satisfy $\Gamma \in \mathcal{C}^{k+1+\alpha, j}(\bar{\Omega})\left(\right.$ resp. $\left.\mathcal{B}^{k+1+\alpha, j}(\bar{\Omega})\right)$. There exist Riemann mappings $R^{\lambda}$ from $\Omega^{\lambda}$ onto $\mathbb{D}$ such that $\left\{R^{\lambda} \circ \Gamma^{\lambda}\right\} \in \mathcal{C}^{k+1+\alpha, j}(\bar{\Omega})$ (resp. $\left.\mathcal{B}^{k+1+\alpha, j}(\bar{\Omega})\right)$. Assume further that $\partial \Omega \in \mathcal{C}^{\omega}$ and $\Gamma \in \mathcal{C}^{\omega}(\bar{\Omega} \times[0,1])$. Then the function $R^{\lambda} \circ \Gamma^{\lambda}(z)$ is real analytic on $\bar{\Omega} \times[0,1]$.

Proof. The proof is standard for the non-parameter case. Since we need it for the next proof, we recall the construction. Fix $a \in \Omega$ and let $a^{\lambda}=\Gamma^{\lambda}(a)$. Let 
$u^{\lambda}\left(z^{\lambda}\right)$ be the harmonic function on $\Omega^{\lambda}$ whose boundary value is $-\log \left|z^{\lambda}-a^{\lambda}\right|$. Let $v^{\lambda}$ be the harmonic conjugate of $u^{\lambda}$ on $\Omega^{\lambda}$ with $v^{\lambda}\left(a^{\lambda}\right)=0$. Then $z^{\lambda} \rightarrow$ $\left(z^{\lambda}-a^{\lambda}\right) e^{u^{\lambda}\left(z^{\lambda}\right)+i v^{\lambda}\left(z^{\lambda}\right)}$ is a Riemann mapping $R^{\lambda}$ sending $\Omega^{\lambda}$ onto $\mathbb{D}$. By Theorem 9.2, we know that $u \in \mathcal{C}^{k+1+\alpha, j}(\bar{\Omega})$. Also,

$$
v^{\lambda}\left(z^{\lambda}\right)=\int_{a^{\lambda}}^{z^{\lambda}}\left(-\partial_{y^{\lambda}} u^{\lambda}\left(z^{\lambda}\right) d x^{\lambda}+\partial_{x^{\lambda}} u^{\lambda}\left(z^{\lambda}\right) d y^{\lambda}\right),
$$

where the path of integration is any $\mathcal{C}^{1}$ curve of the form $\left(x^{\lambda}, y^{\lambda}\right)=\Gamma^{\lambda}(\rho(t))$ with $\rho(0)=a$ and $\rho(1)=z$. Using the integral formula we can verify that $v^{\lambda} \in \mathcal{C}_{*}^{0, j}(\bar{\Omega})$. Then $\partial_{x^{\lambda}} v^{\lambda}=-\partial_{y^{\lambda}} u^{\lambda}$ and $\partial_{y^{\lambda}} v^{\lambda}=\partial_{x^{\lambda}} y^{\lambda}$ imply that $v$ is in $\mathcal{C}^{k+1+\alpha, j}(\bar{\Omega})$. The same argument is valid for the real analytic case.

We now turn to the proof of Theorem 1.2, for which we need a third-order invariant.

Lemma 9.5. Let $\Omega$ be a bounded simply connected domain with $\partial \Omega \in \mathcal{C}^{2+\alpha}$. Assume that at $1, \partial \Omega$ and $\partial \mathbb{D}$ are tangent and have the same exterior normal vector. There exists a unique biholomorphism $S$ from $\bar{\Omega}$ onto $\overline{\mathbb{D}}$ such that $S(1)=1$, $S^{\prime}(1)=1$ and $S^{\prime \prime}(1) \in \mathbf{R}$. Let $R$ be a Riemann mapping from $\bar{\Omega}$ onto $\partial \mathbb{D}$ with $R(1)=1$. Then $S^{\prime \prime}(1)=R^{\prime}(1)^{-1} \operatorname{Re} R^{\prime \prime}(1)+1-R^{\prime}(1)$. Assume further that $\partial \Omega \in \mathcal{C}^{3+\alpha}$. Then at 1 ,

$$
\begin{aligned}
S^{\prime \prime \prime}= & \left(R^{\prime}\right)^{-1}\left\{R^{\prime \prime \prime}+3\left(1-R^{\prime}\right) R^{\prime \prime}+\frac{3}{2}\left(1-R^{\prime}\right)^{2} R^{\prime}\right\} \\
& +\frac{3}{2}\left(R^{\prime}\right)^{-2}\left(\operatorname{Im} R^{\prime \prime}\right)^{2}-3 i\left\{\left(R^{\prime}\right)^{-1} \operatorname{Re} R^{\prime \prime}+\left(1-R^{\prime}\right)\right\}\left(R^{\prime}\right)^{-1} \operatorname{Im} R^{\prime \prime} .
\end{aligned}
$$

Proof. Let $R$ be a Riemann mapping from $\bar{\Omega}$ onto $\overline{\mathbb{D}}$ with $R(1)=1$. The fractional linear transformations that preserve $\mathbb{D}$ and 1 are of the form

$$
L_{a}(z)=\frac{1-\bar{a}}{1-a} \cdot \frac{z-a}{1-\bar{a} z}, \quad|a|<1 .
$$

We have

$$
\begin{gathered}
\left(L_{a} \circ R\right)^{\prime}=\frac{1-\bar{a}}{1-a} \cdot \frac{1-|a|^{2}}{(1-\bar{a} R)^{2}} R^{\prime}, \\
\left(L_{a} \circ R\right)^{\prime \prime}=\frac{1-\bar{a}}{1-a} \cdot \frac{1-|a|^{2}}{(1-\bar{a} R)^{2}}\left(R^{\prime \prime}+\frac{2 \bar{a}\left(R^{\prime}\right)^{2}}{1-\bar{a} R}\right) .
\end{gathered}
$$

Note that $R^{\prime}(1)>0$. We have $R_{1}^{\prime}(1)=1$ for $R_{1}=L_{a} \circ R$ with

$$
a=\frac{1-R^{\prime}(1)}{1+R^{\prime}(1)} \text {. }
$$

We further determine $L_{b}$ under the restriction $1-|b|^{2}=|1-b|^{2}$, i.e. $b=\cos \theta(\cos \theta+$ $i \sin \theta)$ with $\theta \in(-\pi / 2,0) \cup(0, \pi / 2]$. Thus we still have $\left(L_{b} \circ R_{1}\right)^{\prime}(1)=1$. Then $R_{1}(1)=R_{1}^{\prime}(1)=1$ imply that

$$
\left(L_{b} \circ R_{1}\right)^{\prime \prime}(1)=R_{1}^{\prime \prime}(1)-2 i \cot \theta .
$$

Hence, there is a unique $\theta \in(-\pi / 2,0) \cup(0, \pi / 2]$ such that $\left(L_{b} \circ R_{1}\right)^{\prime \prime}(1) \in \mathbf{R}$. At 1 ,

$$
\frac{2 \bar{a} R^{\prime}}{1-\bar{a} R}=1-R^{\prime}, \quad \frac{2 \bar{b}}{1-\bar{b}}=-i \operatorname{Im} R_{1}^{\prime \prime}
$$


Therefore, $S$ equals $L_{b} \circ L_{a} \circ R$. By (9.11), we get at 1 ,

$$
S^{\prime \prime}=\left(L_{b} \circ R_{1}\right)^{\prime \prime}=\operatorname{Re} R_{1}^{\prime \prime}=\left(R^{\prime}\right)^{-1}\left\{\operatorname{Re} R^{\prime \prime}+\left(1-R^{\prime}\right) R^{\prime}\right\} .
$$

Also, $\operatorname{Im} R_{1}^{\prime \prime}(1)=R^{\prime}(1)^{-1} \operatorname{Im} R^{\prime \prime}(1)$. Differentiating (9.11), we obtain at 1 ,

$$
\begin{aligned}
& R_{1}^{\prime \prime \prime}=\left(R^{\prime}\right)^{-1}\left\{R^{\prime \prime \prime}+3\left(1-R^{\prime}\right) R^{\prime \prime}+\frac{3}{2}\left(1-R^{\prime}\right)^{2} R^{\prime}\right\}, \\
& S^{\prime \prime \prime}=\left(L_{b} \circ R_{1}\right)^{\prime \prime \prime}=R_{1}^{\prime \prime \prime}-2 i R_{1}^{\prime \prime} \operatorname{Im} R_{1}^{\prime \prime}-\frac{1}{2}\left(\operatorname{Im} R_{1}^{\prime \prime}\right)^{2}-i \operatorname{Re} R_{1}^{\prime \prime} \operatorname{Im} R_{1}^{\prime \prime} .
\end{aligned}
$$

Expressing $R_{1}^{\prime \prime}(1)$ and $R_{1}^{\prime \prime \prime}(1)$ in $R^{\prime}(1), R^{\prime \prime}(1)$ and $R^{\prime \prime \prime}(1)$ yields the identity.

Proof of Theorem 1.2. We need to find a family of embeddings $\Gamma^{\lambda}$ from $\overline{\mathbb{D}}$ onto $\overline{\Omega^{\lambda}}$ satisfying the following: (a) $\Gamma$ is in $\mathcal{C}^{\infty}(\overline{\mathbb{D}} \times[0,1])$ and real analytic at $(1,0) \in$ $\overline{\mathbb{D}} \times[0,1]$, (b) for any family of Riemann mappings from $\overline{\Omega^{\lambda}}$ onto $\overline{\mathbb{D}}, R \circ \Gamma$ is not real analytic at $(1,0) \in \overline{\mathbb{D}} \times[0,1]$.

It is convenient not to use arc-length. Consider a $\mathcal{C}^{\infty}$ family of simply connected bounded domains $\Omega^{\lambda}$ bounded by

$$
\gamma(t, \lambda)=\rho(t, \lambda) e^{i t}, \quad \rho(0, \lambda)=1=\rho(t, 0),
$$

where $\rho$ is a positive $\mathcal{C}^{\infty}$ function satisfying $\rho(t+2 \pi, \lambda)=\rho(t, \lambda)$. To achieve the analyticity, we will require that $\rho-1$ vanishes near $t=0$ and $\lambda=0$. As complexvalued functions, the outer unit normal vector $\nu(t, \lambda)$ of $\partial \Omega^{\lambda}$ is $-i \gamma^{\prime}(t, \lambda) /\left|\gamma^{\prime}(t, \lambda)\right|$. We have

$$
\begin{gathered}
k(s, t, \lambda)=\frac{1}{\pi} \frac{N(s, t)}{|\gamma(s, \lambda)-\gamma(t, \lambda)|^{2}}, \\
N(s, t, \lambda)=\operatorname{Re}\{\overline{\nu(t, \lambda)(\gamma(t, \lambda)-\gamma(s, \lambda))\} .}
\end{gathered}
$$

In the above and remaining computations, the derivatives are in $s, t$ variables only. The derivatives in $\lambda$ at $\lambda=0$ are indicated in the formal Taylor expansion about $\lambda=0$. For instance,

$$
\gamma(t, \lambda) \sim \sum \gamma_{n}(t) \lambda^{n}, \quad \gamma_{0}(t)=e^{i t} ; \quad k(s, t, \lambda) \sim \sum k_{n}(s, t) \lambda^{n} .
$$

We will derive identities for coefficients of formal power series in $\lambda$, and those identities are therefore valid when they arise from $\mathcal{C}^{\infty}$ functions. We will also denote by $\rho_{(n)}^{(j)}(s)$ the collection of $\partial_{s}^{i} \rho_{l}(s)$ with $i \leq j, l \leq n$ and by $\rho_{(n)}$ the collection of $\rho_{l}$ with $l \leq n$. We will denote by $Q\left(\rho_{(n)}^{(j)}\right)$ a function in $s$ and $t$ which depends on $\rho_{(n)}^{(j)}$ such that

$$
\left|\partial_{s}^{i} \partial_{t}^{l-i} Q\left(\rho_{(n)}^{(j)}\right)(s, t)\right| \leq C\left(n, j, l,\left|\rho_{(n)}\right|_{j+l}\right) \stackrel{\text { def }}{=} C\left(\left|\rho_{(n)}\right|_{j+l}\right) .
$$

To simplify notation, the $Q$ might be different when it reappears.

We express

$$
\begin{gathered}
\gamma^{\prime}(t, \lambda)=i e^{i t}\left(\rho(t, \lambda)-i \rho^{\prime}(t, \lambda)\right), \quad \gamma(t, \lambda)-\gamma(s, \lambda)=B(s, t, \lambda)\left(e^{i t}-e^{i s}\right), \\
B(s, t, \lambda)=\rho(s, \lambda)+(\rho(t, \lambda)-\rho(s, \lambda))\left(1-e^{i(s-t)}\right)^{-1} .
\end{gathered}
$$


Note that $B_{0}(s, t)=1=\left|\gamma^{\prime}(t, 0)\right|$. We also have

$$
\begin{aligned}
N(s, t, \lambda)= & \operatorname{Re}\{\overline{\nu(t, \lambda)}(\gamma(t, \lambda)-\gamma(s, \lambda))\}=\left|e^{i s}-e^{i t}\right|^{2} A(s, t, \lambda), \\
A(s, t, \lambda)= & \left|e^{i s}-e^{i t}\right|^{-2} \operatorname{Re}\left\{\overline{\nu(t, \lambda)}\left(\gamma(t, \lambda)-\gamma(s, \lambda)-i \gamma^{\prime}(t, \lambda)\left(e^{i(s-t)}-1\right)\right)\right\} \\
& +\left|e^{i s}-e^{i t}\right|^{-2}(1-\cos (s-t))\left|\gamma^{\prime}(t, \lambda)\right| .
\end{aligned}
$$

Therefore,

$$
\left|\partial_{s}^{j} \partial_{t}^{k-t} A_{n}(s, t)\right|+\left|\partial_{s}^{j} \partial_{t}^{k-t} B_{n}(s, t)\right| \leq C\left(\left|\rho_{n}\right|_{k+2}\right) .
$$

It is clear that $A(s, t, \lambda), B(s, t, \lambda)$ and $k(s, t, \lambda)=A(s, t, \lambda) /\left(\pi|B(s, t, \lambda)|^{2}\right)$ are $\mathcal{C}^{\infty}$ in $(s, t, \lambda)$. Using $B_{0}=1$, we compute derivatives of $k(s, t, \lambda)$ in $\lambda$ at $\lambda=0$. We find $k_{0}(s, t)=\frac{1}{2 \pi}$. By (9.13) we get $k_{n}(s, t)=Q_{n}\left(\rho_{(n)}^{(2)}\right)(s, t)$, which satisfies (9.12). We also have $d \sigma(t, \lambda)=a(t, \lambda) d t$ with $a(t, \lambda)=\left|\gamma^{\prime}(t, \lambda)\right|$. Then $a_{0}=1$ and $a_{n}=Q\left(\gamma_{(n)}^{\prime}\right)$.

Let $u^{\lambda}\left(z^{\lambda}\right)$ be the harmonic function on $\Omega^{\lambda}$ with boundary value $-\log \left|z^{\lambda}\right|$ on $\partial \Omega^{\lambda}$. To compute $u^{\lambda}$, set $f(s, \lambda)=-\log |\gamma(s, \lambda)|=-\log \rho(s, \lambda)$ and consider

$$
\varphi(s, \lambda)+\int_{0}^{2 \pi} \varphi(t, \lambda) K(s, t, \lambda) a(t, \lambda) d t=f(s, \lambda) .
$$

We have $f_{0}=0$ and $f_{n}(s)=-\rho_{n}(s)+Q\left(\rho_{(n-1)}\right)(s)$. We obtain $\varphi_{0}=0$ and

$$
\varphi_{n}(s)=-\frac{1}{2} \rho_{n}(s)+Q\left(\rho_{(n-1)}^{(2)}\right)(s), \quad n>0 .
$$

Recall that $\varphi$ is real-valued and

$$
(U \varphi)(z, \lambda)=\frac{1}{\pi} \int_{\partial \Omega^{\lambda}} \varphi(s, \lambda) \partial_{\tau^{\lambda}} \arg \left(\zeta^{\lambda}-z^{\lambda}\right) d \sigma^{\lambda}=\operatorname{Re} \mathcal{C}^{\lambda} \varphi .
$$

Let $z=r \in(-1,1)$. We get

$$
\begin{aligned}
\partial_{r}^{j} \partial_{\lambda}^{n} \mathcal{C}^{\lambda} \varphi\left(r^{\lambda}\right)= & \frac{1}{2 \pi i} \sum_{i=0}^{n-1}\left(\begin{array}{c}
n \\
i
\end{array}\right) \int_{0}^{2 \pi} \partial_{\lambda}^{i}(\varphi(s, \lambda)) \partial_{r}^{j} \partial_{\lambda}^{n-i}\left\{\frac{\partial_{s} \gamma(s, \lambda)}{\gamma(s, \lambda)-r^{\lambda}}\right\} d s \\
& +\frac{1}{2 \pi i} \partial_{r}^{j} \int_{\partial \Omega^{\lambda}}\left(\partial_{\lambda}^{n} \varphi(s, \lambda)\right) \frac{d \zeta^{\lambda}}{\zeta^{\lambda}-r^{\lambda}}=I_{1}^{\lambda}\left(r^{\lambda}\right)+I_{2}^{\lambda}\left(r^{\lambda}\right) .
\end{aligned}
$$

We want to emphasize that $\Gamma_{n}(z)$ is not determined by $\rho_{1}, \ldots, \rho_{n}$. Nevertheless, we want to show that, when restricted on the unit circle, $(U \varphi)_{n}$ and all derivatives $\left(\partial_{r}^{i}(U \varphi)_{n}\right)$ depend only on $\rho_{1}, \ldots, \rho_{n}$. For $I_{1}$, we apply Stokes' theorem to transport all derivatives on the Cauchy kernel onto derivatives in $s$. After removing all derivatives on the Cauchy kernel, we set $\lambda=0$ and let $r \rightarrow 1^{-}$. By $\varphi_{0}=0$, (9.14) and a crude estimate on orders of derivatives, we obtain

$$
I_{1}^{\lambda}\left(r^{\lambda}\right)=\mathcal{C}^{0} Q\left(\rho_{(n-1)}^{(n+j+2)}\right)(1), \quad\left|I_{1}^{\lambda}\left(r^{\lambda}\right)\right| \leq C\left(\left|\rho_{(n-1)}\right|_{n+j+3}\right), \quad \lambda=0, r=1 .
$$


To compute $I_{2}^{\lambda}$, we express for $r \in(-1,1)$

$$
\begin{gathered}
\partial_{r} \int_{\partial \Omega^{\lambda}} f^{\lambda}\left(\zeta^{\lambda}\right) \frac{d \zeta^{\lambda}}{\zeta^{\lambda}-r^{\lambda}}=\partial_{r} r^{\lambda} \int_{\partial \Omega^{\lambda}}\left\{\left(\overline{\tau^{\lambda}} \partial_{\tau^{\lambda}}\right) f^{\lambda}\left(\zeta^{\lambda}\right)\right\} \frac{d \zeta^{\lambda}}{\zeta^{\lambda}-r^{\lambda}} \\
\partial_{r}^{j} \int_{\partial \Omega^{\lambda}} f^{\lambda}\left(\zeta^{\lambda}\right) \frac{d \zeta^{\lambda}}{\zeta^{\lambda}-r^{\lambda}}=\left(\partial_{r} r^{\lambda}\right)^{j} \int_{\partial \Omega^{\lambda}}\left\{\left(\overline{\tau^{\lambda}} \partial_{\tau^{\lambda}}\right)^{j} f^{\lambda}\left(\zeta^{\lambda}\right)\right\} \frac{d \zeta^{\lambda}}{\zeta^{\lambda}-r^{\lambda}} \\
+\sum_{i>1, l<j} \partial_{r}^{i} r^{\lambda} Q_{j l}\left(\partial_{r}^{(j-i)} r^{\lambda}\right) \int_{\partial \Omega^{\lambda}}\left\{\left(\overline{\tau^{\lambda}} \partial_{\tau^{\lambda}}\right)^{l} f^{\lambda}\left(\zeta^{\lambda}\right)\right\} \frac{d \zeta^{\lambda}}{\zeta^{\lambda}-r^{\lambda}}
\end{gathered}
$$

Recall that $\gamma_{0}(s)=e^{i t}$. Write $\gamma^{\lambda}\left(e^{i t}\right)=\gamma(t, \lambda)$. We further require that the extension $\Gamma^{\lambda}(z)$ of $\gamma^{\lambda}(z)$ satisfies $\Gamma^{0}(z)=z$. Thus at $(r, \lambda)=(1,0)$, we have $\partial_{r} r^{\lambda}=1$ and $\partial_{r}^{j} r^{\lambda}=0$ for all $j>1$. Set $\lambda=0$, let $r \rightarrow 1^{-}$in $I_{2}^{\lambda}$ and apply the jump formula for Cauchy transform on the unit circle. We get

$$
\begin{aligned}
I_{2}^{0}(1)= & \frac{1}{2 \pi i} \int_{0}^{2 \pi}\left\{\left(-i e^{-i s} \partial_{s}\right)^{j} \varphi_{n}(s)-\left.\left(-i e^{-i t} \partial_{s}\right)^{j} \varphi_{n}(t)\right|_{t=0}\right\} \frac{i e^{i s} d s}{e^{i s}-1} \\
& +\left.\left(\partial_{t} \cdot i e^{-i t}\right)^{r} \varphi_{n}(t)\right|_{t=0} \\
= & -\frac{1}{4 \pi i} \int_{0}^{2 \pi}\left\{\left(-i e^{-i s} \partial_{s}\right)^{j} \rho_{n}(s)-\left.\left(-i e^{-i t} \partial_{t}\right)^{j} \rho_{n}(t)\right|_{t=0}\right\} \frac{i e^{i s} d s}{e^{i s}-1} \\
& -\left.\frac{1}{2}\left(-i e^{-i t} \partial_{t}\right)^{j} \rho_{n}(t)\right|_{t=0}+\mathcal{C}^{0} Q\left(\rho_{(n-1)}^{(2+j)}\right)(1)+Q\left(\rho_{(n-1)}^{(2+j)}\right) \\
= & -\frac{(-1)^{j} j !}{4 \pi i} \int_{0}^{2 \pi} \rho_{n}(s) \frac{i e^{i s} d s}{\left(e^{i s}-1\right)^{j+1}}+Q\left(\rho_{(n-1)}^{(3+j)}\right) .
\end{aligned}
$$

Here $\mathcal{C}^{0}$ stands for the Cauchy transform on the unit circle. Recall in notation (9.12) that we have $\left|Q\left(\rho_{(n-1)}^{(3+j)}\right)\right| \leq C\left(\left|\rho_{(n-1)}\right|_{j+3}\right)$. Here the second-to-last identity is obtained via integration by parts under the additional conditions that $n>0$ and $\rho_{n}$ vanish near $s=0$. Therefore, we get for $n>0$

$$
\partial_{r}^{j}(U \varphi)_{n}(1)=-\frac{(-1)^{j} j !}{4 \pi} \operatorname{Re} \int_{0}^{2 \pi} \rho_{n}(s) \frac{e^{i s} d s}{\left(e^{i s}-1\right)^{j+1}}+Q\left(\rho_{(n-1)}^{(n+6)}\right) .
$$

We use the Riemann mapping $R^{\lambda}$ satisfying $R^{\lambda}(0)=0$ and $\left(R^{\lambda}\right)(1)=1$. Near $(z, \lambda)=(1,0)$, we have $\gamma^{\lambda}(z)=z$ and

$$
R(z, \lambda)=R^{\lambda}\left(\gamma^{\lambda}(z)\right)=z e^{h^{\lambda}(z)}, \quad h^{\lambda}(z)=u^{\lambda}(z)+i v^{\lambda}(z)-u^{\lambda}(1)-i v^{\lambda}(1) .
$$

Here $v^{\lambda}$ is a harmonic conjugate of $u^{\lambda}=U \varphi$. Since $(U \varphi)_{0}=0$, then $(U \varphi)_{0}$ is identically zero. Hence $R_{0}(z)=z$. At $z=1$, we have

$$
\begin{gathered}
R^{\prime}=1+\partial_{r} u^{\lambda}, \quad R^{\prime \prime}=\left(h^{\lambda}\right)^{\prime \prime}+\left(\left(h^{\lambda}\right)^{\prime}\right)^{2}+2\left(h^{\lambda}\right)^{\prime}, \\
R^{\prime \prime \prime}=\left(h^{\lambda}\right)^{\prime \prime \prime}+3\left(h^{\lambda}\right)^{\prime}\left(h^{\lambda}\right)^{\prime \prime}+\left(\left(h^{\lambda}\right)^{\prime}\right)^{3}+3\left(h^{\lambda}\right)^{\prime \prime}+3\left(\left(h^{\lambda}\right)^{\prime}\right)^{2} .
\end{gathered}
$$

We get

$$
\begin{gathered}
\left(R^{\prime}\right)_{0}=1, \quad\left(R^{\prime \prime}\right)_{0}=0, \quad\left(R^{\prime \prime \prime}\right)_{0}=0, \\
\operatorname{Re} R_{n}^{\prime \prime \prime}(1)=\partial_{r}^{3}\left(u^{\lambda}\right)_{n}(1)+3 \partial_{r}^{2}\left(u^{\lambda}\right)_{n}(1)+Q\left(\rho_{(n-1)}^{(3)}\right) .
\end{gathered}
$$

By Lemma 9.5, there exists a unique Riemann mapping $S^{\lambda}$ for $\Omega^{\lambda}$ that satisfies

$$
S^{\lambda}(1)=\left(S^{\lambda}\right)^{\prime}(1)=1, \quad\left(S^{\lambda}\right)^{\prime \prime}(1) \in \mathbf{R} .
$$


Thus, $\left(S^{\lambda}\right)_{n}^{\prime \prime \prime}(1)=R_{n}^{\prime \prime \prime}(1)$ by (9.16)-(9.17) and the last identity in Lemma 9.5. For $n>0$ we obtain

$$
\begin{aligned}
\operatorname{Re} R_{n}^{\prime \prime \prime}(1) & =\frac{3 !}{4 \pi} \operatorname{Re} \int_{0}^{2 \pi} \rho_{n}(s)\left(\frac{e^{i s}}{\left(1-e^{i s}\right)^{4}}+\frac{e^{i s}}{\left(1-e^{i s}\right)^{3}}\right) d s+Q\left(\rho_{n-1}^{(n+6)}\right) \\
& =\frac{3 !}{4 \pi} \int_{0}^{2 \pi} \frac{\rho_{n}(s) \cos (2 s)}{16 \sin ^{4}(s / 2)} d s+Q\left(\rho_{n-1}^{(n+6)}\right) .
\end{aligned}
$$

One can inductively choose $\rho_{n}(s)=\tilde{\rho}_{n}(s) \sin ^{4}(s / 2) \cos (2 s)$ with $\tilde{\rho}_{n} \geq 0$ such that $\rho_{n}(s)=0$ on $|s|<\pi / 2$ and $R_{n}^{\prime \prime \prime}(1)>(n !)^{2}$ for $n>0$. This shows that $\left(S^{\lambda}\right)^{\prime \prime \prime}(1)$ is not real analytic at $\lambda=0$, provided that $\rho_{n}(s)$ can be realized via a family of embeddings $\Gamma^{\lambda}$ satisfying all the requirements. To achieve the latter, we apply a non-parameter version of Lemma 8.1 to the unit disc $\mathbb{D}$ and find $\tilde{\rho}_{n} \in \mathcal{C}^{\infty}(\overline{\mathbb{D}})$ such that $\tilde{\rho}_{n}\left(e^{i s}\right)=\rho_{n}(s)$. Moreover, all $\tilde{\rho}_{n}$ vanish in a fixed neighborhood of $1 \in \overline{\mathbb{D}}$. Applying Lemma 8.3 we find $\tilde{\rho} \in \mathcal{C}^{\infty}(\overline{\mathbb{D}} \times[0,1])$ such that $\tilde{\rho}(z, \lambda)$ vanishes near $(z, \lambda)=(1,0)$ and $\partial_{\lambda}^{n-1} \tilde{\rho}(z, \lambda)=(n-1) ! \tilde{\rho}_{n}(z)$ at $\lambda=0$. Let $\Gamma(z, \lambda)=$ $(1+\lambda \tilde{\rho}(z, \lambda)) z$. As we already mentioned, we can extend $\rho(t, \lambda)$ to be identically 1 near $(1,0) \in \overline{\mathbb{D}} \times I$. Thus $\Gamma(z, \lambda)$ is real analytic near $(1,0)$. Replacing $\Gamma^{\lambda}$ by $\Gamma^{\delta \lambda}$ if necessary, $\Gamma^{\lambda}$ embeds $\overline{\mathbb{D}}$ into $\overline{\Omega^{\lambda}}$ when $\delta>0$ is sufficiently small and $0 \leq \lambda \leq 1$.

We now consider any family of Riemann mappings $R^{\lambda}$ from $\Omega^{\lambda}$ onto $\mathbb{D}$. Assume for the sake of contradiction that $R$ is real analytic at $(1,0) \in \overline{\mathbb{D}} \times[0,1]$. Replace $R^{\lambda}$ by $\overline{R^{\lambda}(1)} R^{\lambda}$. By Lemma $9.5 .\left(S^{\lambda}\right)^{\prime \prime \prime}(1)$ is real analytic at $\lambda=0$, which is a contradiction.

We conclude the paper with a remark on the results and proofs when the domains are fixed and only the boundary values vary with a parameter. In this case we can reduce the solutions to the case without parameter. Recall that the solutions for the Dirichlet and Neumann problems consist of solving the integral equations and estimating the single and double layer potentials via Cauchy transform. When we differentiate integral equations or Cauchy transform in parameter $\lambda$, the kernels are unchanged for fixed domains. The difficulties with the chain rule in our proofs disappear. More specifically, the estimates for the integral equations in Proposition 7.4 (without restriction $k \geq j$ ) extend to spaces of types $\mathcal{B}_{*}$ and $\mathcal{C}_{*}$. The estimates on the layer potentials via Cauchy transform in Proposition 5.2 (without restriction $k \geq j$ ) extend to spaces of types $\mathcal{B}_{*}$ and $\mathcal{C}_{*}$ too. Thus, we have the following.

Proposition 9.6. Let $k, j$ and $l$ be non-negative integers. Assume that $l \leq k+1$ and $0<\alpha<1$. Let $\Omega$ be a bounded domain in the complex plane with $\partial \Omega \in$ $\mathcal{C}^{k+1+\alpha}$. Let $u^{\lambda}$ be harmonic functions on $\Omega$ which are continuous up to boundary. If $u \in \mathcal{B}_{*}^{l+\alpha, j}(\partial \Omega)\left(\operatorname{resp} . \mathcal{C}_{*}^{l+\alpha, j}(\partial \Omega)\right)$, then $u \in \mathcal{B}_{*}^{l+\alpha, j}(\bar{\Omega})\left(\operatorname{resp} . \mathcal{C}_{*}^{l+\alpha, j}(\bar{\Omega})\right)$. If $\int_{\partial \Omega} u^{\lambda} d \sigma=0$ and $\left\{\partial_{\nu} u^{\lambda}\right\}$ is in $\mathcal{B}_{*}^{k+\alpha, j}(\partial \Omega)$ (resp. $\mathcal{C}_{*}^{k+\alpha, j}(\partial \Omega)$ ), then $u \in$ $\mathcal{B}_{*}^{k+1+\alpha, j}(\bar{\Omega})\left(\operatorname{resp} . \mathcal{C}_{*}^{k+1+\alpha, j}(\bar{\Omega})\right)$.

\section{REFERENCES}

[1] L. Bers, Riemann Surfaces (mimeographed lecture notes), New York University (1957-1958).

[2] F. Bertrand, X. Gong and J.-P. Rosay, Common boundary values of holomorphic functions for two-sided complex structures, submitted.

[3] G.B. Folland, Introduction to partial differential equations, second edition. Princeton University Press, Princeton, NJ, 1995. MR.1357411(96h:35001) 
[4] D. Gilbarg and N.S. Trudinger, Elliptic partial differential equations of second order. Reprint of the 1998 edition. Classics in Mathematics. Springer-Verlag, Berlin, 2001. MR.1814364 (2001k:35004)

[5] L. Hörmander, The analysis of linear partial differential operators. I. Distribution theory and Fourier analysis. Springer-Verlag, Berlin, 1990.

[6] O.D. Kellogg, Potential functions on the boundary of their regions of definition, Trans. Amer. Math. Soc. 9(1908), no. 1, 39-50. MR1500801

[7] _ Double distributions and the Dirichlet problem, Trans. Amer. Math. Soc. 9(1908), no. 1, 51-66. MR.1500802

[8] _ Harmonic functions and Green's integral, Trans. Amer. Math. Soc. 13(1912), no. 1, 109-132. MR.1500909

[9] , Foundations of potential theory, Reprint from the first edition of 1929. Die Grundlehren der Mathematischen Wissenschaften, Band 31 Springer-Verlag, Berlin-New York 1967. MR0222317(36:5369)

[10] S.G. Mikhlin, Mathematical Physics: an advanced course, North Holland, Amsterdam, 1970.

[11] C. Miranda, Partial differential equations of elliptic type, Second revised edition. Translated from the Italian by Zane C. Motteler. Ergebnisse der Mathematik und ihrer Grenzgebiete, Band 2. Springer-Verlag, New York-Berlin 1970. MR0284700 (44:1924)

[12] J. Plemelj, Über lineare Randwertaufgaben der Potentialtheorie, I. Teil. Monatsh. Math. Phys. 15(1904), no. 1, 337-411. MR.1547285

[13] Ch. Pommerenke, Boundary behaviour of conformal maps, Grundlehren der Mathematischen Wissenschaften, 299. Springer-Verlag, Berlin, 1992. MR1217706 (95b:30008)

[14] M. Tsuji, Potential theory in modern function theory, Maruzen Co., Ltd., Tokyo, 1959. MR0114894(22:5712)

[15] I.N. Vekua, Generalized analytic functions, Pergamon Press, London-Paris-Frankfurt; Addison-Wesley Publishing Co., Inc., Reading, Mass. 1962. MR.0150320 (27:321)

[16] S.E. Warschawski, Über einen Satz von O.D. Kellogg, Göttinger Nachrichten, Math.-Phys. Klasse, 1932, 73-86.

[17] _ Über das Randverhalten der Ableitung der Abbildungsfunktion bei konformer Abbildung, Math. Z. 35(1932), 321-456. MR.1545302

[18] H. Whitney, Analytic extensions of differentiable functions defined in closed sets, Trans. Amer. Math. Soc. 36(1934), no. 1, 63-89. MR.1501735

Department of Mathematics, University of Wisconsin, Madison, Wisconsin 53706

Current address: Department of Mathematics, University of Vienna, Nordbergstrasse 15, 1090 Vienna, Austria

E-mail address: bertrand@math.wisc.edu

Department of Mathematics, University of Wisconsin, Madison, Wisconsin 53706

E-mail address: gong@math.wisc.edu 